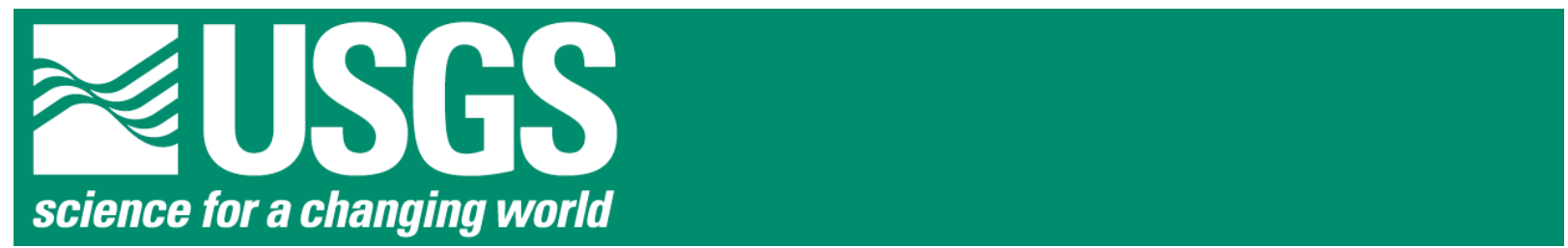

\title{
Sedimentary Properties of Shallow Marine Cores Collected in June and September 2006, Hanalei Bay, Kaua'i, Hawai'i
}

By Amy E. Draut, Michael H. Bothner, Richard L. Reynolds, Olivia C. Buchan, Susan A. Cochran, Michael A. Casso, Sandra M. Baldwin, Harland Goldstein, Jiang Xiao, Michael E. Field, and Joshua B. Logan

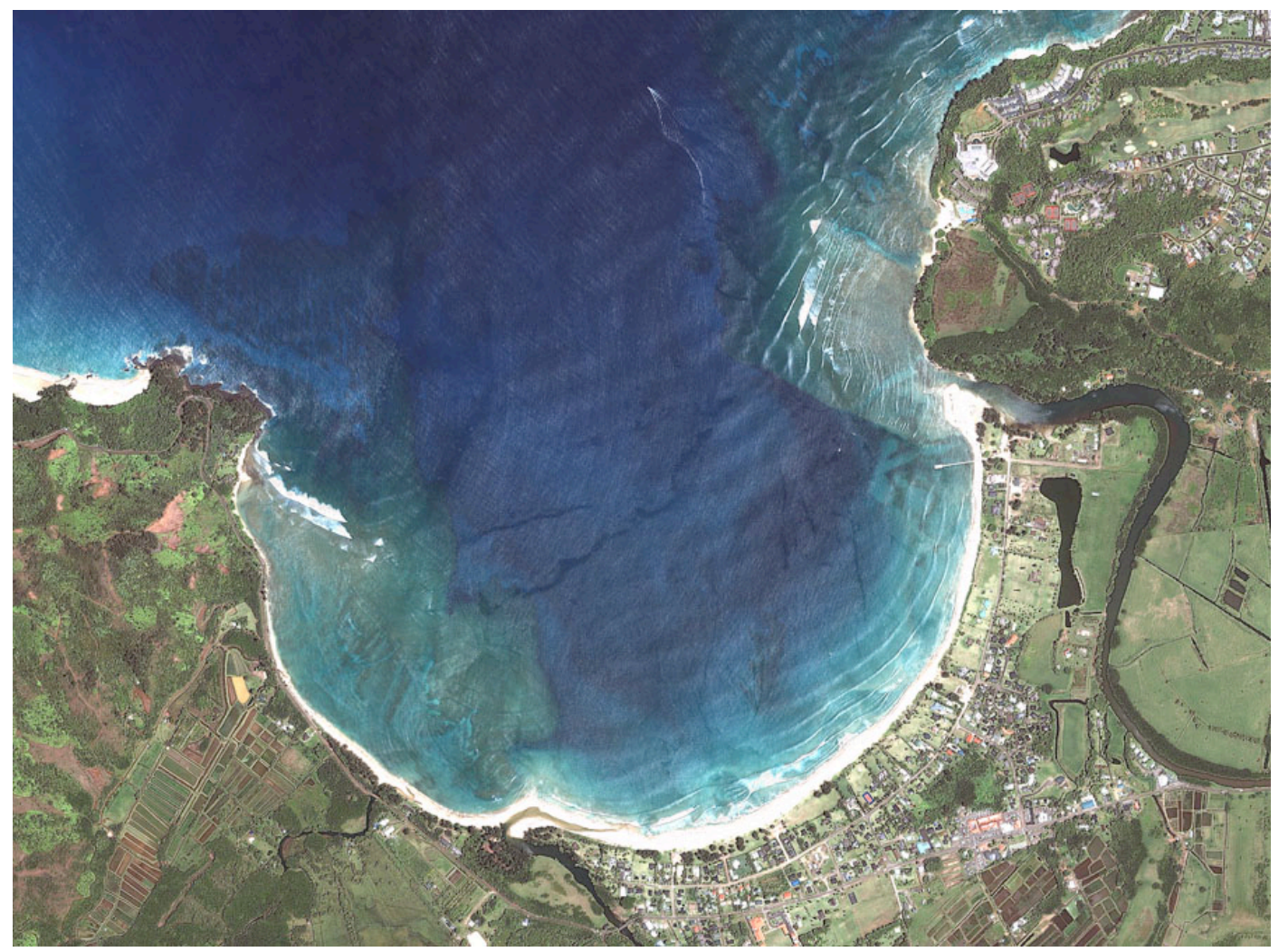

U.S. Department of the Interior

U.S. Geological Survey

Data Series 289 



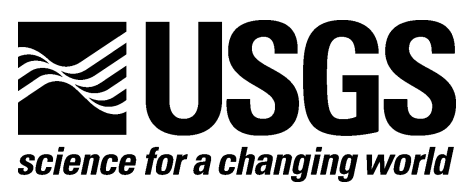

\section{Sedimentary properties of shallow marine cores collected in June and September 2006, Hanalei Bay, Kaua i, Hawai i}

By Amy E. Draut, Michael H. Bothner, Richard L. Reynolds, Olivia C. Buchan, Susan A. Cochran, Michael A. Casso, Sandra M. Baldwin, Harland Goldstein, Jiang Xiao, Michael E. Field, and Joshua B. Logan

Data Series 289

U.S. Department of the Interior U.S. Geological Survey 


\section{U.S. Department of the Interior \\ DIRK KEMPTHORNE, Secretary}

\section{U.S. Geological Survey \\ Mark D. Myers, Director}

U.S. Geological Survey, Reston, Virginia 2007

Revised and reprinted: 2007

For product and ordering information:

World Wide Web: http://www.usgs.gov/pubprod

Telephone: 1-888-ASK-USGS

For more information on the USGS - the Federal source for science about the Earth,

its natural and living resources, natural hazards, and the environment:

World Wide Web: http://www.usgs.gov

Telephone: 1-888-ASK-USGS

Suggested citation:

Draut, A.E., Bothner, M.H., Reynolds, R.L., Buchan, O.C., Cochran, S.A., Casso, M.A., Baldwin, S.M., Goldstein, H., Xiao, J., Field, M.E., and Logan, J.B., 2007, Sedimentary properties of shallow marine cores collected in June and September 2006, Hanalei Bay, Kaua`i, Hawai i: U.S. Geological Survey Data Series 289.

Any use of trade, product, or firm names is for descriptive purposes only and does not imply endorsement by the U.S. Government.

Although this report is in the public domain, permission must be secured from the individual copyright owners to reproduce any copyrighted material contained within this report. 


\section{Contents}

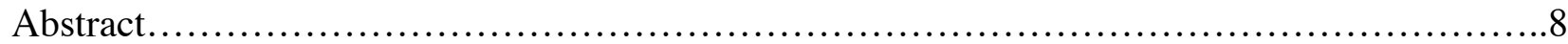

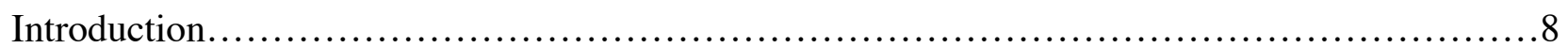

Project Objectives........................................................... 9

Study Area.....................................................................

Isotopes Used as Sediment Tracers.............................................13

Magnetic Properties as Indicators of Terrigenous-Sediment Sources....................14

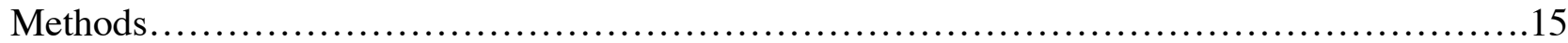

Sediment Collection and Sub-Sampling........................................ 15

Isotope Analysis by Gamma Counting........................................... 17

Grain-Size and Carbonate Analyses.............................................. 17

Magnetic-Property Analyses.................................................25

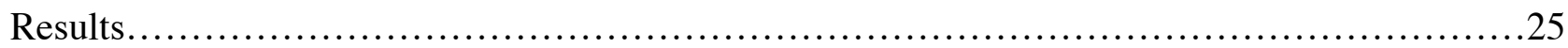

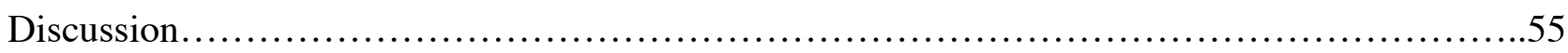

Sedimentary Facies, Isotopic, and Magnetic Signatures...........................55

Effects of the 2006 Hanalei River Floods in Hanalei Bay..............................57

Sources of Flood Sediment in the Hanalei Watershed...................................58

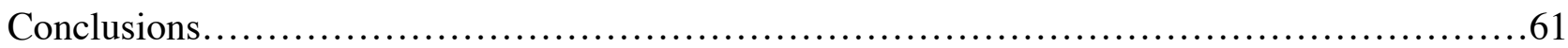

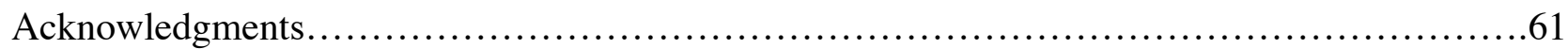

References.......................................................................62 


\section{Figures}

1. Location map of the main eight Hawaiian Islands

2. Locations of sampling sites in Hanalei Bay

3. Water discharge, suspended-sediment discharge, and rainfall in the Hanalei River watershed during water years 2004-2006

4. Proportion of mud in seabed grab samples collected in June and September 2006

5. Sedimentary facies and isotope activity at Site A in June 2006 (Core K0606-A)

6. Sedimentary facies and isotope activity at Site A in September 2006 (Core K0906-A)

7. Sedimentary facies and isotope activity at Site B in June 2006 (Core K0606-B)

8. Sedimentary facies and isotope activity at Site B in September 2006 (Core K0906-B)

9. Sedimentary facies and isotope activity at Site C in June 2006 (Core K0606-C)

10. Sedimentary facies and isotope activity at Site C in September 2006 (Core K0906-C)

11. Sedimentary facies and isotope activity at Site D in June 2006 (Core K0606-D)

12. Sedimentary facies and isotope activity at Site D in September 2006 (Core K0906-D)

13. Sedimentary facies and isotope activity at Site E in June 2006 (Core K0606-E)

14. Sedimentary facies and isotope activity at Site E in September 2006 (Core K0906-E)

15. Sedimentary facies and isotope activity at Site F in June 2006 (Core K0606-F)

16. Sedimentary facies and isotope activity at Site F in September 2006 (Core K0906-F)

17. Sedimentary facies and isotope activity at Site G in June 2006 (Core K0606-G)

18. Sedimentary facies and isotope activity at Site G in September 2006 (Core K0906-G)

19. Sedimentary facies and isotope activity at Site H in June 2006 (Core K0606-H)

20. Sedimentary facies and isotope activity at Site H in September 2006 (Core K0906-H)

21. Sedimentary facies and isotope activity at Site I in June 2006 (Core K0606-I)

22. Sedimentary facies and isotope activity at Site I in September 2006 (Core K0906-I)

23. Magnetic properties measured at Site A in June 2006

24. Magnetic properties measured at Site A in September 2006

25. Magnetic properties measured at Site C in June 2006 
26. Magnetic properties measured at Site C in September 2006

27. Magnetic properties measured at Site I in June 2006

28. Magnetic properties measured at Site I in September 2006

29. Photographs of sediment cores from Hanalei Bay

30. Photographs taken at an automated camera station at the Outer Wall site in Hanalei Bay during the summer of 2006

\section{Tables}

1. Locations and details of sediment cores collected in June and September 2006

2. Locations and details of seabed grab samples collected in June and September 2006

3. Locations and details of sediment traps deployed in 2005 and 2006

4. Locations and details of river-bed samples collected in June and September 2006

5. Locations and details of upland samples collected in September 2006

6. Inventory of ${ }^{7} \mathrm{Be},{ }^{137} \mathrm{Cs}$, and excess ${ }^{210} \mathrm{~Pb}$ at all core sites

\section{Appendices}

1. Sediment logs for cores and river-bed samples collected in June, 2006

2. Sediment $\log$ s for cores and river-bed samples collected in September, 2006

3. Spreadsheet containing all available grain-size, isotopic, and magnetic-property data for all samples collected in 2005 and 2006 


\section{Additional Digital Information}

This report contains material related to the following Infobank Activity ID numbers: S-1-06-KA (for June 2006 field work; ) and S-2-06-KA (for September 2006 field work):

http://walrus.wr.usgs.gov/infobank/s/s106ka/html/s-1-06-ka.meta.html

http://walrus.wr.usgs.gov/infobank/s/s206ka/html/s-2-06-ka.meta.html

For an online PDF version of this report, please see:

http://pubs.usgs.gov/ds/289/

For more information on the U.S. Geological Survey Western Region's Coastal and Marine Geology Team, please see:

http://walrus.wr.usgs.gov/

For more information on the U.S. Geological Survey's Coral Reef Project, please see:

http://coralreefs.wr.usgs.gov/

\section{Contact Information}

General Project Information:

Dr. Michael E. Field (Project Chief)

mfield@usgs.gov

Regarding this Report:

Dr. Amy E. Draut (Research Geologist)

adraut@usgs.gov 


\title{
Sedimentary Properties of Shallow Marine Cores Collected in June and September 2006, Hanalei Bay, Kaua i, Hawai i
}

\begin{abstract}
Sedimentary facies, short-lived isotopes ${ }^{7} \mathrm{Be},{ }^{137} \mathrm{Cs}$, and ${ }^{210} \mathrm{~Pb}$, and magnetic properties of sediment cores in Hanalei Bay, Kaua 'i, Hawai' $i$, were used to assess sediment sources and patterns of deposition associated with seasonal flooding of the Hanalei River. Sediment cores were collected from the seafloor in June and September of 2006 to supplement similar data collected during the summer of 2005. The youngest and thickest terrigenous sediment was observed on the east side of the bay: near the Hanalei River mouth and in a bathymetric depression, known locally as the Black Hole, that acts as a temporary sediment sink. Deposits from floods that occurred between February and April 2006 left flood deposits in the eastern bay that, by June of 2006, were on the order of 10 $\mathrm{cm}$ thick. A flood occurred on August 7, 2006, that was smaller than floods that occurred the previous winter but was a substantial discharge event for the summer season. Deposits from the winter 2006 floods continued to dominate the sedimentary record in the eastern bay through early fall, even after the addition of newer sediment during the August 7 flood; this is consistent with the much higher sediment input of the winter floods compared with the August 7 flood. Broad variations in magnetic grain size and relative magnetite-hematite abundance in several sediment cores indicate many sources of upland terrigenous sediment. As a group, recent flood deposits show much less variation in these properties compared with older deposits, implying either that the 2006 winter-spring flood sediment originated from one or more distinct upland settings, or that substantial mixing of sediment from multiple sources occurred during transport.

Sediment is most readily remobilized and advected out of the bay during winter, when oceanic conditions are energetic. In summer, wave and current measurements made concurrently with this study showed weak currents and little wave energy, indicating that sediment delivered during summer floods most likely remains in the bay until winter storms can remove it. Increased turbidity and sedimentation on corals resulting from floods of the Hanalei River could affect the sustainability of coral reefs and their many associated species. This possibility is of particular concern during summer months when wave energy is low and sediment is not readily remobilized and transported out of the bay. The timing (seasonality) and magnitude of sediment input to the coastal ocean relative to seasonal variations in wave and current energy could have significant ecological consequences for coral-reef communities in the Hawaiian Islands.
\end{abstract}

\section{Introduction}

Analyses of terrestrial sediment delivery to the coral-reef community of Hanalei Bay on the island of Kaua i, Hawai i, USA, were conducted as part of the U.S. Geological Survey (USGS) Coastal and Marine Geology Program's Geological Processes of Coral Reefs Project. The 2006 field studies discussed in this report were made concurrently with measurements of waves, currents, temperature, salinity, and turbidity in Hanalei Bay to be published in a separate report; comparable data collected in 2005 were discussed by Draut and others (2006) and Storlazzi and others (2006). Together, these studies are intended to identify sources and processes that contribute sediment to the bay, in order to understand better the oceanic and sedimentary systems that affect development and health of coral-reef communities there. The ultimate goal of the USGS Coral Reef Project, which is actively involved in similar research in other areas of the Hawaiian Islands, is to understand better the transport mechanisms of sediment, larvae, pollutants, and other water-borne 
substances in coral-reef environments. Assessing the oceanic and sedimentary processes that affect coral-reef communities is a necessary step in identifying potential threats to reefs, which constitute an integral part of the Hawaiian Islands' ecosystem.

Deposition on and burial of corals in Hanalei Bay by fluvial sediment during floods of the Hanalei River, especially in seasons of low wave energy when sediment would not be readily remobilized by waves and removed by currents, could negatively affect coral reefs and many species associated with them (Rogers, 1990; Fabricius and Wolanski, 2000). In addition to the physiological stress imposed by sediment, the coral-reef and near-shore ecosystems may potentially be affected by substances such as fertilizer and other contaminants discharged by the river into the bay.

\section{Project Objectives}

The goal of this component of the USGS Geological Processes of Coral Reef Project is to identify the spatial and temporal distribution of terrestrial sediment in and around the near-shore coral-reef community of Hanalei Bay, Kaua i, Hawai i. Isotope and sedimentary-facies data provide insight into the potential effects of terrestrial sediment delivery patterns around the Hanalei Bay coral reefs, whereas magnetic properties can identify terrigenous sediment entering the bay and possibly its specific sources within the drainage basin. These data are relevant to future watershedrestoration projects proposed by the U.S. Coral Reef Task Force's (USCRTF) Hawaiian Local Action Strategy (LAS) to address land-based-pollution threats to coral reefs in the Hanalei linked watershed-reef system. The work was conducted with the collaboration of the Hanalei Watershed Hui, a local organization that monitors environmental quality in the Hanalei Bay watershed (http://www.hanaleiwatershedhui.org/).

Sediment cores and seabed samples were collected at nine locations within Hanalei Bay during two surveys in June and September, 2006. Additional samples were collected on land within the Hanalei River drainage basin, and from the river itself. Observations of sedimentary facies were used together with measurements of short-lived isotopes ${ }^{7} \mathrm{Be},{ }^{137} \mathrm{Cs}$, and ${ }^{210} \mathrm{~Pb}$ and magnetic properties to trace the thickness and distribution of terrestrial sediment in the bay, and to assess spatial and temporal patterns of sediment deposition and remobilization.

\section{Study Area}

Sediment cores were collected in Hanalei Bay on the northern shore of the island of Kaua $i$, Hawai i (figs. 1, 2). Hanalei Bay covers an area of approximately $4.4 \mathrm{~km}^{2}$. The $25-\mathrm{km}$-long Hanalei River, a designated American Heritage River, is one of the largest rivers in the Hawaiian Islands in terms of water discharge. The river drains an area of $54.4 \mathrm{~km}^{2}$ before debouching into the bay. Its north-facing drainage basin consists of steep-walled, heavily vegetated volcanic ridges and fluvial gorges that drain the island's 1,500-m-high central mountains, a watershed that receives rainfall commonly exceeding $10 \mathrm{~m} \mathrm{yr}^{-1}$. The lowermost $12 \mathrm{~km}$ of the river channel pass through a broad floodplain consisting of middle to late Holocene marine sands overlain by fluvial deposits (Calhoun and Fletcher, 1996) that are used extensively for agriculture. The potential for runoff of sediment and contaminants to affect coral-reef ecology in the bay in the lower river basin is considered a subject of concern by the Hanalei Watershed Hui; it has been suggested that soil erosion caused by agriculture and by feral ungulates in the drainage basin could increase the sediment load in the Hanalei River. The steep topography of the drainage basin is subject to landslides that deliver variable amounts of sediment to the river, with an estimated total sediment yield of $140 \pm 55 \mathrm{Mg}$ $\mathrm{km}^{-2} \mathrm{yr}^{-1}$ (Calhoun and Fletcher, 1999). Calhoun and Fletcher (1999) estimated that approximately 


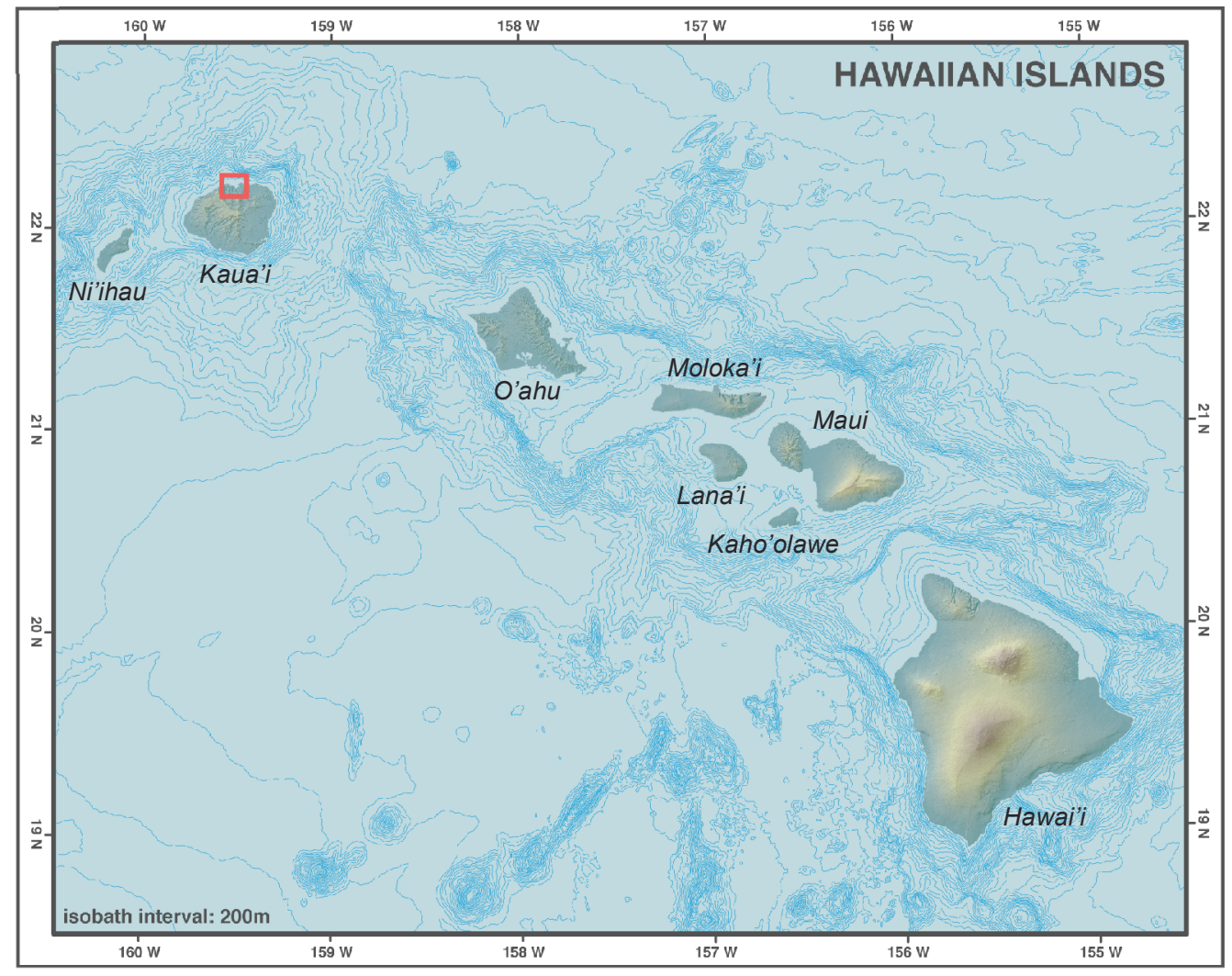

Figure 1. Location map showing the major Hawaiian Islands. Hanalei Bay, on the northern shore of Kaua'i, is shown in the red box. 
Figure 2. Locations of sediment-core sites in Hanalei Bay. Stations occupied in June and September 2006 for this study (yellow circles) are identified by station letter (A-I). River Site 4 (RS4) is also marked; other river sites are outside this map area. Red circles indicate the locations of sediment traps and oceanographic instrument packages deployed by Storlazzi and others (2006, in prep.): SC, South Central site; IW, Inner Wall site; OW, Outer Wall site; NE, Northeast Dobie site; CRAMP, Coral Reef Assessment and Monitoring Program site also used by University of Hawai'i. Bathymetry in this image was interpolated from SHOALS lidar data; dark-blue areas indicate no bathymetric data. The isobath interval is $5 \mathrm{~m}$. 

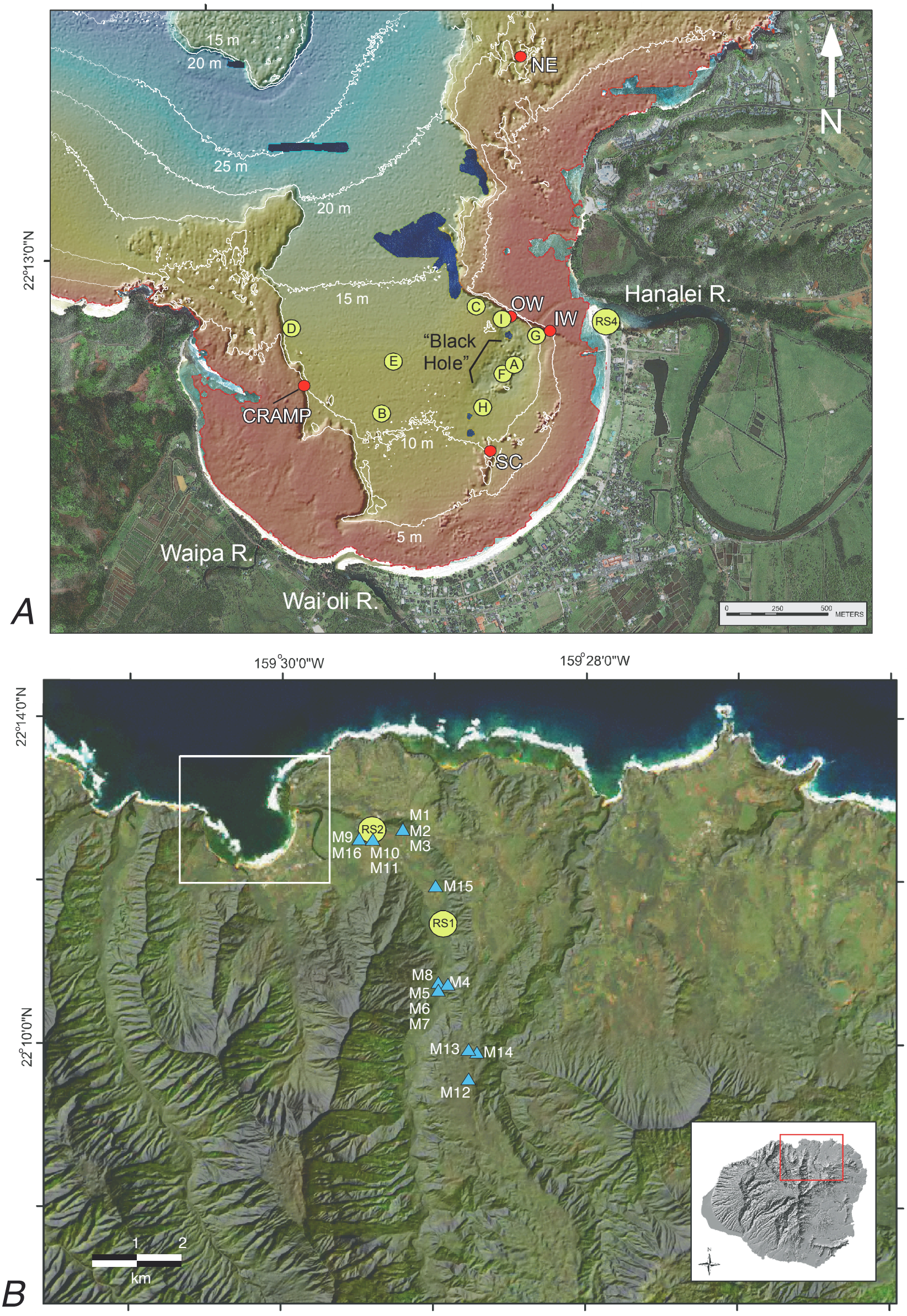
30 percent of the annual fluvial sediment load is deposited on the coastal floodplain, while 70 percent $\left(1.76 \times 10^{4} \mathrm{Mg}\right)$ is delivered to Hanalei Bay.

The Hanalei River enters Hanalei Bay from the east (fig. 2); the smaller Waioli and Waipa perennial streams also discharge intermittently into the bay's southern and western sides. Submarine groundwater discharge at the western side of the bay can occur even in the absence of surface stream flow there (Storlazzi and others, 2006). The physical environment of the bay in summer is dominated by northeast to easterly trade winds, accompanied by low wave energy. From June through August 2005, significant wave heights were $<1 \mathrm{~m}$ within the bay itself with periods of 2.5-5.9 seconds, driven dominantly by northeast trade winds (Storlazzi and others, 2006); conditions during summer 2006 were similar. Winter conditions, typically beginning in October, are characterized by a North Pacific swell regime that produces wave heights of $2-5 \mathrm{~m}$ with periods of 12-20 seconds (Moberly and Chamberlain, 1964) that approach the bay typically from the northwest. Occasional low-pressure systems, including hurricanes, approach Kaua i, but usually make landfall on the southern coast, away from Hanalei Bay.

Current meters deployed during the summer of 2005 (Storlazzi and others, 2006) indicated weak currents in the bay from early June through late August, with net near-surface flow entering the bay in the east and flowing out of the bay in the west. Near-bed current velocities indicated opposite, cyclonic (counter-clockwise) flow. Waves and currents in the western part of the bay were more energetic than in the east. Currents of $5 \mathrm{~cm} \mathrm{~s}^{-1}$ were measured near the surface at the CRAMP (Coral Reef Assessment and Monitoring Project, a study site established by the University of Hawai i) site compared to $2 \mathrm{~cm} \mathrm{~s}^{-1}$ near the surface at the Outer Wall site (Storlazzi and others, 2006, see fig. 2A), a difference attributed to more direct exposure to trade winds on the western side of the bay.

The sea floor of Hanalei Bay consists of marine carbonate sediment and terrigenous siliciclastic material derived from the basaltic highlands of the Hanalei River basin (Calhoun and others, 2002). The center of the bay is largely free of indurated coral-reef substrate, with a flat sea floor at typically 10-18 m water depth. On the eastern side of the bay, $500 \mathrm{~m}$ offshore of the river mouth, is a broad depression $\sim 15 \mathrm{~m}$ deep known as the Black Hole because terrestrial sediment and dark organic matter obscures visibility in the water column (fig. $2 A$ ). The fringing reef inside Hanalei Bay hosts ecological communities that were, in recent studies of the bay's marine biological diversity, considered to be relatively healthy. Hanalei Bay is unusual among Hawaiian coral communities in having increased its live coral cover since the mid-1990s (Friedlander and others, 1997, 2005).

\section{Isotopes Used as Sediment Tracers}

This study utilizes three radioisotopes: ${ }^{7} \mathrm{Be},{ }^{137} \mathrm{Cs}$, and ${ }^{210} \mathrm{~Pb}$. These isotopes have been used in previous studies of near-shore and inner-shelf sedimentation to assess accumulation rates and sources. All three isotopes are particle-reactive and adsorb readily onto the surfaces of sediment grains. Beryllium-7 has been shown to be especially useful for identifying episodic seasonal flood deposits near shore (Allison and others, 2000). Lead-210 and ${ }^{137} \mathrm{Cs}$ have been used together in numerous coastal and lacustrine settings to estimate accumulation rates and deposition age of sediment (Duursma and Gross, 1971; Nittrouer and others, 1979; Smith and Ellis, 1982; Buesseler and Benitez, 1994; Allison and others, 1998, 2000; Jaeger and Nittrouer, 1995; Kuehl and others, 1995; Goodbred and Kuehl, 1998; Draut and others, 2005).

The first tracer, ${ }^{7} \mathrm{Be}$, is a naturally occurring isotope with a 53-day half-life that forms in the atmosphere by cosmic-ray spallation of nitrogen and oxygen. The production of cosmogenic ${ }^{7} \mathrm{Be}$ is 
controlled by insolation, and so the flux of ${ }^{7} \mathrm{Be}$ from the atmosphere to the earth's surface has a latitudinal and seasonal dependence (Olsen and others, 1986; Baskaran and others, 1993). After it becomes associated with airborne particles and is deposited on land, ${ }^{7} \mathrm{Be}$ rapidly becomes incorporated into vegetation and soil. In contrast to the slow rate at which ${ }^{7} \mathrm{Be}$ can be delivered to the sea floor via settling of particles through the marine water column (which may take longer than its activity remains measurable), ${ }^{7} \mathrm{Be}$ is concentrated in terrestrial sediment carried to the coastal ocean by river discharge. An appreciable inventory of this isotope can therefore accumulate in fluvial deposits near shore (Canuel and others, 1990; Allison and others, 2000). The presence of ${ }^{7} \mathrm{Be}$ in near-shore sediment indicates that the sediment had been exposed to the atmosphere within the past eight months, or approximately five half-lives; therefore, it is useful for detecting recently deposited fluvial sediment.

Cesium-137 is an isotope with a 30 -year half-life and a non-constant source function. It was first introduced to the environment during nuclear-weapon testing that began in the 1950s and peaked in $1963 ;{ }^{137} \mathrm{Cs}$ ceased to be added to the atmosphere after the 1972 atmospheric nuclear testing ban, with the exception of additions caused by the 1979 and 1986 nuclear accidents at Three Mile Island and Chernobyl, respectively. Because ${ }^{137} \mathrm{Cs}$ has been almost entirely removed from the atmosphere by rainfall, it is now introduced to the marine environment primarily via sediment that has been eroded from land and discharged by rivers into the ocean (for example, Smith and Ellis, 1982). As with ${ }^{7} \mathrm{Be}$, the supply of ${ }^{137} \mathrm{Cs}$ to shallow marine settings is controlled largely by fluvial sedimentation. Measurable activity of ${ }^{137} \mathrm{Cs}$ indicates that some component of the sediment has been in contact with an atmospheric or fluvial source more recently than the 1950 s, when this isotope was first introduced to the environment. If sediment has accumulated undisturbed since that time, the ${ }^{137} \mathrm{Cs}$ profile with sediment depth may mirror the history of atmospheric fallout, and so can be used to infer accumulation rates (Livingston and Bowen, 1979; Miller and Heit, 1986).

The third isotope, ${ }^{210} \mathrm{~Pb}$, is a naturally occurring daughter product in the ${ }^{238} \mathrm{U}$ decay chain, with a half-life of 22.3 years. Excess ${ }^{210} \mathrm{~Pb}$ is defined as the ${ }^{210} \mathrm{~Pb}$ activity above (in excess of) the equilibrium activity supported by activity of parent and grandparent isotopes in the ${ }^{238} U$ decay series in the same sediment sample. The excess ${ }^{210} \mathrm{~Pb}$ activity is the difference between the total ${ }^{210} \mathrm{~Pb}$ and the supported ${ }^{210} \mathrm{~Pb}$. Supported ${ }^{210} \mathrm{~Pb}$, the amount of ${ }^{210} \mathrm{~Pb}$ in sediment produced in place by continuous ${ }^{226} \mathrm{Ra}$ decay (via ${ }^{222} \mathrm{Rn}$, with a 3.8-day half-life), is identified by measurement of ${ }^{214} \mathrm{~Pb}$ (half-life 26.8 minutes), an intermediate isotope between ${ }^{222} \mathrm{Rn}$ and ${ }^{210} \mathrm{~Pb}$ that is assumed to be in secular equilibrium with ${ }^{210} \mathrm{~Pb}$. Because the half-life of ${ }^{226} \mathrm{Ra}$ is long (1,622 years), supported ${ }^{210} \mathrm{~Pb}$ is produced in marine sediment by ${ }^{226} \mathrm{Ra}$ decay for thousands of years after its deposition and isolation from other ${ }^{210} \mathrm{~Pb}$ sources. In the coastal marine environment of the Hawaiian Islands, adjacent to clear Pacific Ocean water, the primary source of excess ${ }^{210} \mathrm{~Pb}$ is the decay of ${ }^{226} \mathrm{Ra}$ dissolved in seawater. Other sources include fallout to surface water following its production in the atmosphere from the decay of ${ }^{222} \mathrm{Rn}$ gas and input by fluvial discharge. The presence of excess ${ }^{210} \mathrm{~Pb}$ characterizes sediment deposited within the past 100 years, or five half-lives. In many undisturbed marine sediment profiles, the excess ${ }^{210} \mathrm{~Pb}$-activity profile can be used to model rates of sediment accumulation and mixing (for example, Nittrouer and others, 1979; Crusius and others, 2004).

\section{Magnetic Properties as Indicators of Terrigenous-Sediment Sources}

Magnetic-property measurements determine the amount, type, and grain size of irontitanium oxide minerals in sediment samples. Iron-titanium oxide minerals (hematite $\left[\mathrm{Fe}_{2} \mathrm{O}_{3}\right]$, ilmenite $\left[\mathrm{FeTiO}_{3}\right]$, magnetite $\left[\mathrm{Fe}_{3} \mathrm{O}_{4}\right]$, and rutile $\left[\mathrm{TiO}_{2}\right]$ ) are a ubiquitous component of mafic volcanic rocks. These minerals are not produced by the processes that form coral or carbonate of 
any type, so any Fe-Ti oxide minerals within Hanalei Bay must have been derived from nearby island (terrestrial) sources. Magnetic properties of bay sediments record detrital input from the watershed and may reflect sources and landscape settings of upland sediment. Magnetic mineral types and magnetic grain sizes (domain states) vary among different volcanic rocks according to magmatic composition and cooling history (Reynolds and others, 1990; Rosenbaum and others, 1991, 1994), variations that are commonly inherited in derived soils and volcanogenic sediments. Subsequent changes in magnetic properties result from weathering of soil and sediment (oxidation of magnetite to hematite; development of pedogenic Fe oxides) depending on geomorphic, hydrologic, and microbial setting. Magnetic analyses of sediment are used in conjunction with facies and isotopic analyses to provide information about available terrigenous sediment sources, aspects of sediment transport, such as mineral sorting, and, possibly, the stability of land surfaces within the watershed.

Magnetic properties measured during this study included (1) magnetic susceptibility (MS), a measure of the amount of all magnetic minerals in a sample. Contributions to MS come primarily from ferromagnetic minerals, such as magnetite and titanomagnetite, and from paramagnetic minerals (certain Fe-Ti oxide minerals, such as ilmenite, and Fe-bearing silicates, such as olivine). Diamagnetic effects counteract positive MS from Fe-bearing minerals and can be caused by components such as biogenic silica and water in wet sediment; (2) frequency-dependent magnetic susceptibility (FDMS), a measure of superparamagnetic (ultra-fine grained, <30 nm) magnetite and (or) maghemite, commonly produced via pedogenic processes; (3) isothermal remanent magnetization (IRM), laboratory-induced magnetization used to measure the quantity of magnetite sufficiently large (magnetic grain size greater than about $30 \mathrm{~nm}$ ) to carry remanence. This parameter is measured as $\mathrm{IRM}_{0.3 \mathrm{~T}}$, the magnetization acquired by a sample after exposure to a 0.3 Tesla (T) magnetic field; and (4) anhysteretic remanent magnetization (ARM), laboratory-induced magnetization that is a measure of magnetite content sensitive to single-domain (fine magnetic grain size) magnetite.

The ratio of ARM to MS provides a concentration-independent indicator of magnetic grain size (domain state of magnetite). Similarly, the ratio of ARM to IRM provides a concentrationindependent assessment of magnetic grain size, but does not have the complication that MS is sensitive to Fe-bearing minerals other than magnetite. Magnetic grain size, which may not indicate the physical size of a magnetite particle, reflects the magnetic domain structure of magnetic minerals, thereby providing information about origins of these minerals. ARM/IRM values increase as the magnetic grain size of magnetite decreases, and are particularly sensitive to single domain (SD) and small pseudo-single domain (PSD) magnetic grain sizes. A so-called S parameter (ratio of IRM acquired at $0.3 \mathrm{~T}$ to IRM acquired at $1.2 \mathrm{~T}$ ) represents the relative proportions of magnetite and hematite in a sample. An $S$ parameter value of 1 indicates a population of all magnetite, whereas lower values reflect increasingly higher hematite content.

\section{Methods}

\section{Sediment Collection and Sub-Sampling}

Sediment cores were collected with a diver-operated hand-held coring unit at nine sites in Hanalei Bay. Seabed samples were collected by divers at all core sites and at five additional locations. Core collection occurred in June and September 2006 at sites chosen to provide representative spatial coverage around the bay (fig. 2, table 1). Three of nine core sites were in the Black Hole (Sites A, F, and I), two sites were along the coral wall on the eastern side of the bay 
near the Hanalei River mouth (Sites C and G), two sites were in the central part of the bay (Sites B and $\mathrm{E}$ ), one site was in the southeastern part of the bay (Site $\mathrm{H})$, and one site was along the coral wall at the northwestern side of the bay (Site D). Seabed sample locations were chosen such that spatial coverage of the seafloor could be increased above what was attained by the more laborintensive coring effort (table 2).

The diver-operated corer uses a slide hammer to drive a 10.7-cm-diameter polycarbonate core barrel into the sea floor and can collect cores up to $\sim 0.8 \mathrm{~m}$ long, depending on the consolidation of the sediment. A check valve within the slide hammer-core barrel adapter provides suction to retain sediment while the barrel is pulled out of the sediment. The bottom of the core barrel is sealed as soon as possible by inserting a piston. Core lengths recovered during this study typically ranged from 30 to $50 \mathrm{~cm}$ (table 1). At sites of uniform unconsolidated sand, core recovery was complicated by the movement of water through the sediment during insertion of the piston.

After core collection, sediment was extruded from the core barrel by using a jack to push the piston and sediment through the barrel. Sedimentary facies and textures were described qualitatively and sampled during extrusion. The top $22 \mathrm{~cm}$ of each core typically were sampled in $2-\mathrm{cm}$ intervals; below $22 \mathrm{~cm}$, sample thicknesses typically alternated between $2 \mathrm{~cm}$ and $8 \mathrm{~cm}$. Sampling resolution was higher in cores with notable facies variations and lower in cores containing homogenous sediment. Sediment from the edges of each sample was discarded to avoid sampling material that had been smeared, obscuring its original depth, as the core barrel was driven into the sea floor or extruded from the barrel.

Seabed samples (table 2) comprised grab samples of the uppermost $\sim 5 \mathrm{~cm}$ of sediment at the seafloor and were collected by divers using acrylic pipes $20 \mathrm{~cm}$ long with an 8-cm diameter. After recovery, samples were homogenized and washed through a $63-\mu$ sieve to separate sand-sized from mud-sized particles. The sand fraction was dried in an oven at $65^{\circ} \mathrm{C}$ and weighed. The mud fraction was allowed to settle overnight. Excess water was then decanted or siphoned off, and the residual mud slurry was suctioned through a $0.45-\mu$ filter. Sediment-laden filters were oven-dried at $65^{\circ} \mathrm{C}$ and weighed, to allow calculation of the relative proportions, by mass, of sand and mud in each sample.

Two types of sediment traps were deployed in Hanalei Bay in June 2006, to collect suspended sediment from bay water during the summer (table 3). Tube traps that were mounted on instrument tripods (Storlazzi and others, in prep.) consisted of a 30-cm-long clear plastic tube with an internal diameter of $6.7 \mathrm{~cm}$. A honeycomb-shaped baffle was placed in the top of each tube trap to reduce turbulence and minimize disturbance by aquatic organisms (see Bothner and others, 2006). Programmable rotating sediment traps also were deployed, at two locations in the bay (fig. 2; table 3). Rotating traps used a 20-cm-diameter, 75-cm-long collection cylinder equipped with a funnel in the lower $15 \mathrm{~cm}$ of the cylinder to direct settling sediment into each of $21500-\mathrm{ml}$ plastic bottles. Sampling bottles were mounted on a carousel that rotated a new bottle under the funnel every 4.5 days. The two time-series traps were deployed such that the trap opening was positioned $\sim 1.3 \mathrm{~m}$ above the sea floor (Bothner and others, 2006).

In addition to sediment cores, seabed grab samples, and suspended-sediment samples collected within Hanalei Bay, sediment grab samples $(0-5 \mathrm{~cm}$ thick) were obtained from the Hanalei River bottom at three locations in June and September 2006 (table 4). Facies at the three river sites (RS1, RS2, and RS4) were described, and, along with selected core samples, were analyzed on gamma counters to allow comparison of isotope activity in the river sediment with that in the bay. 
One sample of river water was collected at the Hanalei River mouth on September 6, 2006, during a discharge of $\sim 4 \mathrm{~m}^{3} \mathrm{~s}^{-1}$; suspended-solid and colloidal material were isolated from that sample.

Terrestrial sediment samples were collected from 16 locations within the Hanalei drainage basin in September, 2006 (fig. 2B, table 5). Upland sample locations targeted a variety of sedimentary environments: agricultural fields on the floodplain where taro crops are grown, nonagricultural grassland on the floodplain, exposures along a dirt road that runs parallel to the river, and forested areas along the river corridor. Sampling locations spanned the lowermost $\sim 10 \mathrm{~km}$ of the river.

\section{Isotope Analysis by Gamma Counting}

Gamma-ray activity was analyzed in selected sediment samples to evaluate activity levels of ${ }^{7} \mathrm{Be},{ }^{137} \mathrm{Cs}$, and ${ }^{210} \mathrm{~Pb}$. Gamma-ray activity provides a straightforward and efficient means to measure the radioactive isotope content in sediment (Gäggler and others, 1976). Each isotope emits gamma radiation at a characteristic frequency associated with its decay. Because detection by this method allows detection of multiple frequencies simultaneously, the activities of all desired isotopes are assessed in one counting session.

Laboratory analyses were performed at the USGS laboratory in Woods Hole, Mass. The wet sediment samples were homogenized and sub-sampled. Aliquots weighing approximately $100 \mathrm{~g}$ were freeze-dried to facilitate disaggregation. Samples of known weight (on a calculated salt-free basis) were analyzed on planar germanium detectors (Canberra Industries, Inc., model GS2020S) for $48-96$ hours (until the ${ }^{210} \mathrm{~Pb}$ standard error was within $3 \%$ ). Activity levels of ${ }^{7} \mathrm{Be}$, ${ }^{137} \mathrm{Cs}$, and ${ }^{210} \mathrm{~Pb}$ were measured by using net counts of the 477.6, 661.6, and $46.5 \mathrm{keV}$ peaks, respectively. Excess ${ }^{210} \mathrm{~Pb}$ activity was calculated from independent measurement of ${ }^{214} \mathrm{~Pb}$ at $352 \mathrm{keV}$ (Livingston and Bowen, 1979; Joshi, 1987). The efficiency of the detectors over the energy range 46.5-661.6 keV was calibrated by using EPA standard pitchblende ore in the same range of geometry as the samples. Calibration of the detectors specifically for ${ }^{137} \mathrm{Cs}(661.6 \mathrm{keV})$ was done with a standard solution from Isotope Products Laboratory. The activities of ${ }^{7} \mathrm{Be},{ }^{137} \mathrm{Cs}$, and excess

${ }^{210} \mathrm{~Pb}$ were decay-corrected to the date of core collection. A correction for self-absorption on all of the isotopes was made using the method of Cutshall and others (1983). The accuracy of these results was confirmed by analyses of standard reference materials, which yielded agreement within three percent of certified results.

Isotope inventories (total activity per $\mathrm{cm}^{2}$ of sea-floor area at each core site) were calculated for the cores by multiplying the measured activity (in disintegrations per minute per dry gram of sediment) in each depth interval by the estimated bulk density $\left(\mathrm{g} \mathrm{cm}^{-3}\right)$ and by the core section thickness $(\mathrm{cm})$. The products were summed over the entire core length. Sediment bulk density was estimated using a measured weight per unit volume of disaggregated, freeze-dried sediment. This procedure assumes that the unconsolidated sandy sediment was not compacted appreciably (observed to be the case during core recovery and sub-sampling).

\section{Grain-Size and Carbonate Analyses}

Grain-size analysis and percent-carbonate determination for the samples were performed in the USGS sediment lab in Menlo Park, Calif. Methods for grain-size analysis were modified from Carver (1971) and Folk (1974) and standardized by the USGS Western Region Coastal and Marine Geology Team. 


\begin{tabular}{|c|c|c|c|c|c|c|c|}
\hline Site & $\begin{array}{l}\text { Latitude } \\
\text { (WGS84) }\end{array}$ & $\begin{array}{l}\text { Longitude } \\
\text { (WGS84) }\end{array}$ & Date & Core & $\begin{array}{l}\text { Water Depth } \\
\text { (m) }\end{array}$ & $\begin{array}{l}\text { Core Length } \\
\qquad(\mathrm{cm})\end{array}$ & Location \\
\hline $\mathrm{A}$ & 22.21231 & -159.50139 & $6 / 1 / 06$ & K0606-A & 17.1 & 55.0 & Black Hole \\
\hline $\mathrm{B}$ & & & $9 / 10 / 06$ & K0906-B & 11.9 & 46.0 & \\
\hline \multirow[t]{2}{*}{$\mathrm{C}$} & 22.21481 & -159.50326 & $6 / 1 / 06$ & K0606-C & 12.5 & 47.0 & NE side of bay \\
\hline & & & $9 / 10 / 06$ & K0906-C & 12.8 & 51.0 & \\
\hline \multirow[t]{2}{*}{$E$} & 22.21238 & -159.50790 & $6 / 3 / 06$ & K0606-E & 12.2 & 40.0 & Middle of bay \\
\hline & & & $9 / 10 / 06$ & K0906-E & 12.8 & 46.0 & \\
\hline \multirow[t]{2}{*}{$\mathrm{F}$} & 22.21209 & -159.50144 & $6 / 3 / 06$ & K0606-F & 15.5 & 51.0 & Black Hole \\
\hline & & & $9 / 12 / 06$ & K0906-F & 15.5 & 72.0 & \\
\hline \multirow[t]{2}{*}{$\mathrm{G}$} & 22.21346 & -159.50066 & $6 / 2 / 06$ & K0606-G & 10.1 & 33.0 & Reef wall near river mouth \\
\hline & & & $9 / 11 / 06$ & K0906-G & 10.1 & 41.0 & \\
\hline
\end{tabular}

Table 1. Locations and details of sediment cores collected in June and September 2006. 


\section{June 2006}

\begin{tabular}{|c|c|c|c|}
\hline Sample & Latitude & Longitude & Date \\
\hline & & & \\
\hline KA-0606-21 & 22.21481 & -159.50326 & $06 / 01 / 06$ \\
\hline KA-0606-22 & 22.21231 & -159.50139 & $06 / 01 / 06$ \\
\hline KA-0606-23 & 22.21400 & -159.51272 & $06 / 02 / 06$ \\
\hline KA-0606-24 & 22.20991 & -159.50843 & $06 / 02 / 06$ \\
\hline KA-0606-25 & 22.21074 & -159.51153 & $06 / 02 / 06$ \\
\hline KA-0606-26 & 22.21238 & -159.50790 & $06 / 02 / 06$ \\
\hline KA-0606-28 & 22.21346 & -159.50066 & $06 / 03 / 06$ \\
\hline KA-0606-29 & 22.21243 & -159.50476 & $06 / 03 / 06$ \\
\hline KA-0606-30 & 22.21514 & -159.50330 & $06 / 04 / 06$ \\
\hline KA-0606-31 & 22.21411 & -159.50201 & $06 / 04 / 06$ \\
\hline KA-0606-32 & 22.21056 & -159.50219 & $06 / 04 / 06$ \\
\hline KA-0606-33 & 22.20885 & -159.50340 & $06 / 04 / 06$ \\
\hline KA-0606-35 & 22.21368 & -159.50006 & $06 / 05 / 06$ \\
\hline
\end{tabular}

\section{September 2006}

\begin{tabular}{|c|c|c|c|}
\hline Sample & Latitude & Longitude & Date \\
\hline & & & \\
\hline KA-0906-01 & 22.21074 & -159.51153 & $09 / 09 / 06$ \\
\hline KA-0906-02 & 22.20891 & -159.50335 & $09 / 09 / 06$ \\
\hline KA-0906-03 & 22.21409 & -159.50122 & $09 / 09 / 06$ \\
\hline KA-0906-04 & 22.20991 & -159.50843 & $09 / 10 / 06$ \\
\hline KA-0906-05 & 22.21481 & -159.50326 & $09 / 10 / 06$ \\
\hline KA-0906-06 & 22.21400 & -159.51272 & $09 / 10 / 06$ \\
\hline KA-0906-07 & 22.21238 & -159.50790 & $09 / 10 / 06$ \\
\hline KA-0906-08 & 22.21074 & -159.51153 & $09 / 10 / 06$ \\
\hline KA-0906-09 & 22.21231 & -159.50139 & $09 / 11 / 06$ \\
\hline KA-0906-10 & 22.21209 & -159.50144 & $09 / 11 / 06$ \\
\hline KA-0906-11 & 22.21346 & -159.50066 & $09 / 11 / 06$ \\
\hline KA-0906-12 & 22.21056 & -159.50219 & $09 / 12 / 06$ \\
\hline KA-0906-13 & 22.21411 & -159.50201 & $09 / 12 / 06$ \\
\hline KA-0906-14 & 22.21243 & -159.50476 & $09 / 12 / 06$ \\
\hline
\end{tabular}

Table 2. Locations and details of seabed grab samples collected in June and September 2006. 


\begin{tabular}{|c|c|c|c|c|c|c|c|c|c|c|c|c|}
\hline Station Name & Sample ID & $\begin{array}{l}\text { Latitude } \\
\text { (WGS84) }\end{array}$ & $\begin{array}{c}\text { Longitude } \\
\text { (WGS84) }\end{array}$ & $\begin{array}{l}\text { Date/Time of } \\
\text { Deployment }\end{array}$ & $\begin{array}{l}\text { Date/Time of } \\
\text { Recovery }\end{array}$ & Trap Type & $\begin{array}{c}\text { Water } \\
\text { Depth } \\
\text { (m) }\end{array}$ & $\begin{array}{c}\text { Height } \\
\text { Above } \\
\text { Bottom } \\
\text { (m) }\end{array}$ & $\begin{array}{l}\text { Area of Trap } \\
\text { Opening }\left(m^{2}\right)\end{array}$ & $\begin{array}{l}\text { Calculated } \\
\text { Total Salt- } \\
\text { Free Dry } \\
\text { Sed. in Trap } \\
\text { (g) }\end{array}$ & $\begin{array}{l}\text { Percent } \\
\text { Salt in } \\
\text { Dried } \\
\text { Aliquots }\end{array}$ & $\begin{array}{c}\text { Laliculateu } \\
\text { Salt-Free Dry } \\
\text { Sed. } \\
\text { Collection } \\
\text { Rate } \\
\left(\mathrm{g} / \mathrm{m}^{2} / \text { day }\right)\end{array}$ \\
\hline South-Central & S-1-05-KA-001* & 22.20807 & -159.50329 & 6/6/05 12:00 PM & 8/23/05 12:00 PM & $\begin{array}{c}67 \mathrm{~mm} \text { ID } \times 30 \mathrm{~cm} \\
\text { Tube }\end{array}$ & 10 & 0.4 & 0.0035257 & 19.13 & 1.39 & 69.57 \\
\hline NE Dobie & S-1-05-KA-002* & 22.22601 & -159.50132 & 6/6/05 12:00 PM & 8/23/05 12:00 PM & $\begin{array}{c}67 \mathrm{~mm} \mathrm{ID} \times 30 \mathrm{~cm} \\
\text { Tube }\end{array}$ & 10 & 0.4 & 0.0035257 & 88.07 & 0.96 & 320.23 \\
\hline Outer Wall & S-1-05-KA-003* & 22.21407 & -159.50116 & 6/6/05 12:00 PM & 8/23/05 12:00 PM & $\begin{array}{c}67 \mathrm{~mm} \mathrm{ID} \times 30 \mathrm{~cm} \\
\text { Tube }\end{array}$ & 10 & 0.4 & 0.0035257 & 11.36 & 1.72 & 41.31 \\
\hline CRAMP & S-1-05-KA-004* & 22.21084 & -159.51176 & 6/6/05 12:00 PM & 8/23/05 12:00 PM & $\begin{array}{c}67 \mathrm{~mm} \text { ID } \times 30 \mathrm{~cm} \\
\text { Tube }\end{array}$ & 10 & 0.4 & 0.0035257 & 22.20 & 0.38 & 80.71 \\
\hline South-Central & SC-1 & 22.20891 & -159.50335 & 6/7/06 12:00 PM & 6/11/06 8:57 PM & $\begin{array}{c}\text { 21-Bottle Time } \\
\text { Series Trap }\end{array}$ & 10 & 1.4 & 0.0317309 & 13.23 & 5.20 & 95.38 \\
\hline South-Central & SC-2 & 22.20891 & -159.50335 & 6/11/06 8:57 PM & 6/16/06 5:54 AM & $\begin{array}{l}\text { 21-Bottle Time } \\
\text { Series Trap }\end{array}$ & 10 & 1.4 & 0.0317309 & 21.08 & 5.60 & 151.92 \\
\hline South-Central & SC-3 & 22.20891 & -159.50335 & 6/16/06 5:54 AM & 6/20/06 2:51 PM & $\begin{array}{l}\text { 21-Bottle Time } \\
\text { Series Trap }\end{array}$ & 10 & 1.4 & 0.0317309 & 1.71 & 13.29 & 12.32 \\
\hline South-Central & SC-4 & 22.20891 & -159.50335 & 6/20/06 2:51 PM & 6/24/06 11:48 PM & $\begin{array}{l}\text { 21-Bottle Time } \\
\text { Series Trap }\end{array}$ & 10 & 1.4 & 0.0317309 & 3.93 & 8.24 & 28.30 \\
\hline South-Central & SC-5 & 22.20891 & -159.50335 & 6/24/06 11:48 PM & 6/29/06 8:45 AM & $\begin{array}{c}\text { 21-Bottle Time } \\
\text { Series Trap }\end{array}$ & 10 & 1.4 & 0.0317309 & 7.73 & 7.91 & 55.73 \\
\hline South-Central & SC-6 & 22.20891 & -159.50335 & 6/29/06 8:46 AM & 7/3/06 5:42 PM & $\begin{array}{l}\text { 21-Bottle Time } \\
\text { Series Trap }\end{array}$ & 10 & 1.4 & 0.0317309 & 2.92 & 12.12 & 21.03 \\
\hline South-Central & SC-7 & 22.20891 & -159.50335 & 7/3/06 5:43 PM & 7/8/06 2:39 AM & $\begin{array}{l}\text { 21-Bottle Time } \\
\text { Series Trap }\end{array}$ & 10 & 1.4 & 0.0317309 & 2.05 & 17.33 & 14.75 \\
\hline South-Central & SC-8 & 22.20891 & -159.50335 & $7 / 8 / 06$ 2:40 AM & 7/12/06 11:37 AM & $\begin{array}{l}\text { 21-Bottle Time } \\
\text { Series Trap }\end{array}$ & 10 & 1.4 & 0.0317309 & 2.46 & 14.40 & 17.70 \\
\hline South-Central & SC-9 & 22.20891 & -159.50335 & 7/12/06 11:37 AM & 7/16/06 8:34 PM & $\begin{array}{l}\text { 21-Bottle Time } \\
\text { Series Trap }\end{array}$ & 10 & 1.4 & 0.0317309 & 6.42 & 9.82 & 46.25 \\
\hline South-Central & SC-10 & 22.20891 & -159.50335 & 7/16/06 8:34 PM & 7/21/06 5:31 AM & $\begin{array}{l}\text { 21-Bottle Time } \\
\text { Series Trap }\end{array}$ & 10 & 1.4 & 0.0317309 & 6.82 & 12.24 & 49.16 \\
\hline South-Central & SC-11 & 22.20891 & -159.50335 & $7 / 21 / 065: 31 \mathrm{AM}$ & 7/25/06 2:28 PM & $\begin{array}{l}\text { 21-Bottle Time } \\
\text { Series Trap }\end{array}$ & 10 & 1.4 & 0.0317309 & 20.70 & 7.91 & 149.18 \\
\hline South-Central & SC-12 & 22.20891 & -159.50335 & $7 / 25 / 06$ 2:28 PM & 7/29/06 11:25 PM & $\begin{array}{l}\text { 21-Bottle Time } \\
\text { Series Trap }\end{array}$ & 10 & 1.4 & 0.0317309 & 5.93 & 11.14 & 42.76 \\
\hline South-Central & SC-13 & 22.20891 & -159.50335 & 7/29/06 11:26 PM & 8/3/06 8:22 AM & $\begin{array}{l}\text { 21-Bottle Time } \\
\text { Series Trap }\end{array}$ & 10 & 1.4 & 0.0317309 & 13.39 & 12.51 & 96.50 \\
\hline South-Central & SC-14 & 22.20891 & -159.50335 & 8/3/06 8:23 AM & 8/7/06 5:19 PM & $\begin{array}{l}\text { 21-Bottle Time } \\
\text { Series Trap }\end{array}$ & 10 & 1.4 & 0.0317309 & 6.12 & 13.21 & 44.12 \\
\hline South-Central & SC-15 & 22.20891 & -159.50335 & 8/7/06 5:20 PM & 8/12/06 2:17 AM & $\begin{array}{l}\text { 21-Bottle Time } \\
\text { Series Trap }\end{array}$ & 10 & 1.4 & 0.0317309 & 2.97 & 16.23 & 21.38 \\
\hline South-Central & SC-16 & 22.20891 & -159.50335 & $8 / 12 / 06$ 2:17 AM & 8/16/06 11:14 AM & $\begin{array}{c}\text { 21-Bottle Time } \\
\text { Series Trap }\end{array}$ & 10 & 1.4 & 0.0317309 & 2.91 & 16.39 & 21.00 \\
\hline South-Central & SC-17 & 22.20891 & -159.50335 & 8/16/06 11:14 AM & 8/20/06 8:11 PM & $\begin{array}{l}\text { 21-Bottle Time } \\
\text { Series Trap }\end{array}$ & 10 & 1.4 & 0.0317309 & 2.08 & 20.16 & 14.98 \\
\hline South-Central & SC-18 & 22.20891 & -159.50335 & 8/20/06 8:11 PM & 8/25/06 5:08 AM & $\begin{array}{l}\text { 21-Bottle Time } \\
\text { Series Trap }\end{array}$ & 10 & 1.4 & 0.0317309 & 2.77 & 17.51 & 19.96 \\
\hline South-Central & SC-19 & 22.20891 & -159.50335 & 8/25/06 5:08 AM & 8/29/06 2:05 PM & $\begin{array}{c}\text { 21-Bottle Time } \\
\text { Series Trap }\end{array}$ & 10 & 1.4 & 0.0317309 & 3.23 & 19.86 & 23.30 \\
\hline South-Central & SC-20 & 22.20891 & -159.50335 & 8/29/06 2:05 PM & 9/2/06 11:02 PM & $\begin{array}{l}\text { 21-Bottle Time } \\
\text { Series Trap }\end{array}$ & 10 & 1.4 & 0.0317309 & 2.40 & 20.18 & 17.32 \\
\hline South-Central & SC-21 & 22.20891 & -159.50335 & 9/2/06 11:03 PM & 9/7/06 7:59 AM & $\begin{array}{l}\text { 21-Bottle Time } \\
\text { Series Trap }\end{array}$ & 10 & 1.4 & 0.0317309 & 6.53 & 13.24 & 47.10 \\
\hline Outer Wall & WS- 1 & 22.21403 & -159.50120 & 6/7/06 12:00 PM & 6/11/06 8:57 PM & $\begin{array}{l}\text { 21-Bottle Time } \\
\text { Series Trap }\end{array}$ & 10 & 1.4 & 0.0317309 & 5.13 & 11.42 & 36.99 \\
\hline Outer Wall & WS-2 & 22.21403 & -159.50120 & 6/11/06 8:57 PM & 6/16/06 5:54 AM & $\begin{array}{l}\text { 21-Bottle Time } \\
\text { Series Trap }\end{array}$ & 10 & 1.4 & 0.0317309 & 14.91 & 9.02 & 107.47 \\
\hline Outer Wall & WS-3 & 22.21403 & -159.50120 & 6/16/06 5:54 AM & 6/20/06 2:51 PM & $\begin{array}{l}\text { 21-Bottle Time } \\
\text { Series Trap }\end{array}$ & 10 & 1.4 & 0.0317309 & 5.33 & 12.44 & 38.41 \\
\hline Outer Wall & WS-4 & 22.21403 & -159.50120 & 6/20/06 2:51 PM & 6/24/06 11:48 PM & $\begin{array}{l}\text { 21-Bottle Time } \\
\text { Series Trap }\end{array}$ & 10 & 1.4 & 0.0317309 & 6.83 & 12.50 & 49.22 \\
\hline Outer Wall & WS-5 & 22.21403 & -159.50120 & 6/24/06 11:48 PM & 6/29/06 8:45 AM & $\begin{array}{l}\text { 21-Bottle Time } \\
\text { Series Trap }\end{array}$ & 10 & 1.4 & 0.0317309 & 6.27 & 13.09 & 45.18 \\
\hline Outer Wall & WS-6 & 22.21403 & -159.50120 & 6/29/06 8:46 AM & 7/3/06 5:42 PM & $\begin{array}{l}\text { 21-Bottle Time } \\
\text { Series Trap }\end{array}$ & 10 & 1.4 & 0.0317309 & 5.99 & 11.62 & 43.19 \\
\hline Outer Wall & WS-7 & 22.21403 & -159.50120 & 7/3/06 5:43 PM & 7/8/06 2:39 AM & $\begin{array}{l}\text { 21-Bottle Time } \\
\text { Series Trap }\end{array}$ & 10 & 1.4 & 0.0317309 & 6.79 & 12.27 & 48.92 \\
\hline Outer Wall & WS-8 & 22.21403 & -159.50120 & $7 / 8 / 062: 40 \mathrm{AM}$ & 7/12/06 11:37 AM & $\begin{array}{c}\text { 21-Bottle Time } \\
\text { Series Trap }\end{array}$ & 10 & 1.4 & 0.0317309 & 9.88 & 11.85 & 71.22 \\
\hline Outer Wall & WS-9 & 22.21403 & $\mid-159.50120$ & 7/12/06 11:37 AM & 7/16/06 8:34 PM & $\begin{array}{l}\text { 21-Bottle Time } \\
\text { Series Trap }\end{array}$ & 10 & 1.4 & 0.0317309 & 7.65 & 12.58 & 55.15 \\
\hline Outer Wall & WS- 10 & 22.21403 & -159.50120 & 7/16/06 8:34 PM & 7/21/06 5:31 AM & $\begin{array}{l}\text { 21-Bottle Time } \\
\text { Series Trap }\end{array}$ & 10 & 1.4 & 0.0317309 & 5.76 & 12.93 & 41.52 \\
\hline Outer Wall & WS-11 & 22.21403 & $\mid-159.50120$ & 7/21/06 5:31 AM & 7/25/06 2:28 PM & $\begin{array}{l}\text { 21-Bottle Time } \\
\text { Series Trap }\end{array}$ & 10 & 1.4 & 0.0317309 & 6.92 & 11.96 & 49.91 \\
\hline Outer Wall & WS-12 & 22.21403 & -159.50120 & 7/25/06 2:28 PM & 7/29/06 11:25 PM & $\begin{array}{l}\text { 21-Bottle Time } \\
\text { Series Trap }\end{array}$ & 10 & 1.4 & 0.0317309 & 6.09 & 14.45 & 43.87 \\
\hline Outer Wall & WS-13 & 22.21403 & -159.50120 & 7/29/06 11:26 PM & 8/3/06 8:22 AM & $\begin{array}{c}\text { 21-Bottle Time } \\
\text { Series Trap }\end{array}$ & 10 & 1.4 & 0.0317309 & 10.75 & 12.34 & 77.50 \\
\hline Outer Wall & WS-14 (bulk) & 22.21403 & $\mid-159.50120$ & 8/3/06 8:23 AM & 8/7/06 5:19 PM & $\begin{array}{l}\text { 21-Bottle Time } \\
\text { Series Trap }\end{array}$ & 10 & 1.4 & 0.0317309 & 70.89 & $\mathrm{n} / \mathrm{a}$ & 510.94 \\
\hline Outer Wall & $\begin{array}{c}\text { K0606;WS-14;0- } \\
4.8 \mathrm{~cm}\end{array}$ & 22.21403 & -159.50120 & & & $\begin{array}{l}\text { 21-Bottle Time } \\
\text { Series Trap }\end{array}$ & 10 & 1.4 & 0.0317309 & 16.98 & 14.74 & $\mathrm{n} / \mathrm{a}$ \\
\hline Outer Wall & $\begin{array}{c}\text { K0606;WS-14;4.8- } \\
9.6 \mathrm{~cm}\end{array}$ & 22.21403 & -159.50120 & & & $\begin{array}{l}\text { 21-Bottle Time } \\
\text { Series Trap }\end{array}$ & 10 & 1.4 & 0.0317309 & 20.34 & 13.59 & $\mathrm{n} / \mathrm{a}$ \\
\hline Outer Wall & $\begin{array}{c}\text { K0606;WS-14;9.6- } \\
14.4 \mathrm{~cm}\end{array}$ & 22.21403 & -159.50120 & & & $\begin{array}{l}\text { 21-Bottle Time } \\
\text { Series Trap }\end{array}$ & 10 & 1.4 & 0.0317309 & 16.32 & 15.64 & $\mathrm{n} / \mathrm{a}$ \\
\hline Outer Wall & $\begin{array}{c}\text { K0606;WS-14;14.4- } \\
18.3 \mathrm{~cm}\end{array}$ & 22.21403 & -159.50120 & & & $\begin{array}{l}\text { 21-Bottle Time } \\
\text { Series Trap }\end{array}$ & 10 & 1.4 & 0.0317309 & 13.02 & 14.77 & $\mathrm{n} / \mathrm{a}$ \\
\hline Outer Wall & $\begin{array}{c}\text { K0606;WS-14;18.3- } \\
19.3 \mathrm{~cm}\end{array}$ & 22.21403 & $\mid-159.50120$ & & & $\begin{array}{l}\text { 21-Bottle Time } \\
\text { Series Trap }\end{array}$ & 10 & 1.4 & 0.0317309 & 4.23 & 13.50 & $\mathrm{n} / \mathrm{a}$ \\
\hline Outer Wall & WS-(15-21) ** & 22.21403 & $\mid-159.50120$ & 8/7/06 5:20 PM & 9/7/06 7:59 AM & $\begin{array}{l}\text { 21-Bottle Time } \\
\text { Series Trap }\end{array}$ & 10 & 1.4 & 0.0317309 & 152.92 & $\mathrm{n} / \mathrm{a}$ & 157.44 \\
\hline Inner Wall & IWT1 (bulk) & 22.21368 & $\mid-159.50006$ & 6/6/06 12:00 PM & 9/5/06 12:00 PM & $\begin{array}{c}30 \mathrm{~cm} \times 67 \mathrm{~mm} \\
\text { diam. Tube }\end{array}$ & 10 & 0.4 & 0.0035257 & 68.38 & $\mathrm{n} / \mathrm{a}$ & 213.14 \\
\hline Inner Wall & K0606;IWT1;0-3cm & 22.21368 & -159.50006 & & & $\begin{array}{c}30 \mathrm{~cm} \times 67 \mathrm{~mm} \\
\text { diam. Tube }\end{array}$ & 10 & 0.4 & 0.0035257 & 24.25 & 12.66 & $\mathrm{n} / \mathrm{a}$ \\
\hline
\end{tabular}




\begin{tabular}{|c|c|c|c|c|c|c|c|c|c|c|c|c|}
\hline Inner Wall & $\begin{array}{c}\text { K0606;IWT1;3- } \\
6.1 \mathrm{~cm}\end{array}$ & 22.21368 & -159.50006 & & & $\begin{array}{c}30 \mathrm{~cm} \times 67 \mathrm{~mm} \\
\text { diam. Tube }\end{array}$ & 10 & 0.4 & 0.0035257 & 44.13 & 8.01 & $\mathrm{n} / \mathrm{a}$ \\
\hline Outer Wall & OWT1 (bulk) & 22.21406 & -159.50121 & 6/6/06 12:00 PM & 9/5/06 12:00 PM & $\begin{array}{c}30 \mathrm{~cm} \times 67 \mathrm{~mm} \\
\text { diam. Tube }\end{array}$ & 10 & 0.4 & 0.0035257 & 56.63 & $\mathrm{n} / \mathrm{a}$ & 176.52 \\
\hline Outer Wall & $\begin{array}{l}\text { K0606;OWT1;0- } \\
2.6 \mathrm{~cm}\end{array}$ & 22.21406 & -159.50121 & & & $\begin{array}{c}30 \mathrm{~cm} \times 67 \mathrm{~mm} \\
\text { diam. Tube }\end{array}$ & 10 & 0.4 & 0.0035257 & 19.37 & 13.02 & n/a \\
\hline Outer Wall & $\begin{array}{c}\text { K0606;OWT1;2.6- } \\
6.1 \mathrm{~cm}\end{array}$ & 22.21406 & -159.50121 & & & $\begin{array}{c}30 \mathrm{~cm} \times 67 \mathrm{~mm} \\
\text { diam. Tube }\end{array}$ & 10 & 0.4 & 0.0035257 & 37.26 & 9.52 & n/a \\
\hline South-Central & SCRT1 (bulk) & 22.20881 & -159.50339 & 6/6/06 12:00 PM & 9/6/06 12:00 PM & $\begin{array}{c}30 \mathrm{~cm} \times 67 \mathrm{~mm} \\
\text { diam. Tube }\end{array}$ & 10 & 0.4 & 0.0035257 & 206.25 & $\mathrm{n} / \mathrm{a}$ & 635.86 \\
\hline South-Central & $\begin{array}{c}\text { K0606;SCRT1;0- } \\
4.5 \mathrm{~cm}\end{array}$ & 22.20881 & -159.50339 & & & $\begin{array}{c}30 \mathrm{~cm} \times 67 \mathrm{~mm} \\
\text { diam. Tube }\end{array}$ & 10 & 0.4 & 0.0035257 & 57.69 & 6.75 & $\mathrm{n} / \mathrm{a}$ \\
\hline South-Central & $\begin{array}{l}\text { K0606;SCRT1;4.5- } \\
\text { 8cm }\end{array}$ & 22.20881 & -159.50339 & & & $\begin{array}{c}30 \mathrm{~cm} \times 67 \mathrm{~mm} \\
\text { diam. Tube }\end{array}$ & 10 & 0.4 & 0.0035257 & 101.14 & 3.18 & n/a \\
\hline South-Central & $\begin{array}{c}\text { K0606;SCRT1;8- } \\
\text { 9cm }\end{array}$ & 22.20881 & -159.50339 & & & $\begin{array}{c}30 \mathrm{~cm} \times 67 \mathrm{~mm} \\
\text { diam. Tube }\end{array}$ & 10 & 0.4 & 0.0035257 & 20.62 & $\mathrm{n} / \mathrm{a}$ & n/a \\
\hline South-Central & $\begin{array}{c}\text { K0606;SCRT1;9- } \\
10 \mathrm{~cm}\end{array}$ & 22.20881 & -159.50339 & & & $\begin{array}{c}30 \mathrm{~cm} \times 67 \mathrm{~mm} \\
\text { diam. Tube }\end{array}$ & 10 & 0.4 & 0.0035257 & 26.80 & 2.15 & n/a \\
\hline CRAMP & CRAMPT1 (bulk) & 22.21076 & -159.51166 & 6/6/06 12:00 PM & 9/6/06 12:00 PM & $\begin{array}{c}30 \mathrm{~cm} \times 67 \mathrm{~mm} \\
\text { diam. Tube }\end{array}$ & 10 & 0.4 & 0.0035257 & 140.58 & $\mathrm{n} / \mathrm{a}$ & 433.39 \\
\hline CRAMP & $\begin{array}{c}\text { K0606;CRAMPT1;0 } \\
\text { 4cm }\end{array}$ & 22.21076 & -159.51166 & & & $\begin{array}{c}30 \mathrm{~cm} \times 67 \mathrm{~mm} \\
\text { diam. Tube }\end{array}$ & 10 & 0.4 & 0.0035257 & 61.80 & 6.12 & $\mathrm{n} / \mathrm{a}$ \\
\hline CRAMP & $\begin{array}{c}\text { K0606;CRAMPT1;4. } \\
7.6 \mathrm{~cm}\end{array}$ & 22.21076 & -159.51166 & & & $\begin{array}{c}30 \mathrm{~cm} \times 67 \mathrm{~mm} \\
\text { diam. Tube }\end{array}$ & 10 & 0.4 & 0.0035257 & 78.77 & 4.94 & n/a \\
\hline NE Dobie & NEDT1 (bulk) *** & 22.22604 & -159.50133 & 6/6/06 12:00 PM & 9/6/06 12:00 PM & $\begin{array}{c}30 \mathrm{~cm} \times 67 \mathrm{~mm} \\
\text { diam. Tube }\end{array}$ & 10 & 0.4 & 0.0035257 & 1143.91 & $\mathrm{n} / \mathrm{a}$ & 3526.63 \\
\hline NE Dobie & $\begin{array}{l}\text { K0606;NEDT1;0- } \\
\text { 5cm }\end{array}$ & 22.22604 & -159.50133 & & & $\begin{array}{c}30 \mathrm{~cm} \times 67 \mathrm{~mm} \\
\text { diam. Tube }\end{array}$ & 10 & 0.4 & 0.0035257 & 219.14 & $\mathrm{n} / \mathrm{a}$ & n/a \\
\hline NE Dobie & $\begin{array}{c}\text { K0606;NEDT1;5- } \\
10.5 \mathrm{~cm}\end{array}$ & 22.22604 & -159.50133 & & & $\begin{array}{c}30 \mathrm{~cm} \times 67 \mathrm{~mm} \\
\text { diam. Tube }\end{array}$ & 10 & 0.4 & 0.0035257 & 203.35 & 1.54 & $\mathrm{n} / \mathrm{a}$ \\
\hline NE Dobie & $\begin{array}{c}\text { K0606;NEDT1;10.5- } \\
\text { 16cm }\end{array}$ & 22.22604 & -159.50133 & & & $\begin{array}{c}30 \mathrm{~cm} \times 67 \mathrm{~mm} \\
\text { diam. Tube }\end{array}$ & 10 & 0.4 & 0.0035257 & 241.72 & 1.40 & $\mathrm{n} / \mathrm{a}$ \\
\hline NE Dobie & \begin{tabular}{|c|} 
K0606;NEDT1;16- \\
$21 \mathrm{~cm}$
\end{tabular} & 22.22604 & -159.50133 & & & $\begin{array}{c}30 \mathrm{~cm} \times 67 \mathrm{~mm} \\
\text { diam. Tube }\end{array}$ & 10 & 0.4 & 0.0035257 & 233.00 & 1.55 & $\mathrm{n} / \mathrm{a}$ \\
\hline NE Dobie & \begin{tabular}{|c|} 
K0606;NEDT1;21- \\
23cm
\end{tabular} & 22.22604 & -159.50133 & & & $\begin{array}{c}30 \mathrm{~cm} \times 67 \mathrm{~mm} \\
\text { diam. Tube }\end{array}$ & 10 & 0.4 & 0.0035257 & 110.57 & 1.71 & n/a \\
\hline NE Dobie & $\begin{array}{c}\text { K0606;NEDT1;23- } \\
26.1 \mathrm{~cm}\end{array}$ & 22.22604 & -159.50133 & & & $\begin{array}{c}30 \mathrm{~cm} \times 67 \mathrm{~mm} \\
\text { diam. Tube }\end{array}$ & 10 & 0.4 & 0.0035257 & 136.13 & 1.63 & $\mathrm{n} / \mathrm{a}$ \\
\hline South-Central & SCT1* & 22.20891 & -159.50335 & 6/6/06 10:00 AM & 9/7/06 10:00 AM & $\begin{array}{c}60 \mathrm{~cm} \times 67 \mathrm{~mm} \\
\text { diam. Tube on } \\
\text { Time Series Frame }\end{array}$ & 10 & 1.4 & 0.0035257 & 15.43 & 10.48 & 47.07 \\
\hline Outer Wall & WST1* & 22.21403 & -159.50120 & 6/5/06 10:25 AM & 9/7/06 11:25 AM & $\begin{array}{c}60 \mathrm{~cm} \times 67 \mathrm{~mm} \\
\text { diam. Tube on } \\
\text { Time Series Frame }\end{array}$ & 10 & 1.4 & 0.0035257 & 23.36 & 14.63 & 70.45 \\
\hline
\end{tabular}

* fraction $>1 \mathrm{~mm}$ removed, usually negligible.

** Carousel kept rotating but funnel plugged after bottle \#14. Mass of sediment estimated from "bathtub ring" in funnel. Collection rate

is averaged over the remainder of the deployment period, but most was probably associated with the single flood event at bottle 14.

*** Trap was nearly full. Erosion of surface may have occured. Collection rate may be a minimum.

Table 3. Locations and details of sediment traps deployed in 2005 and 2006. 


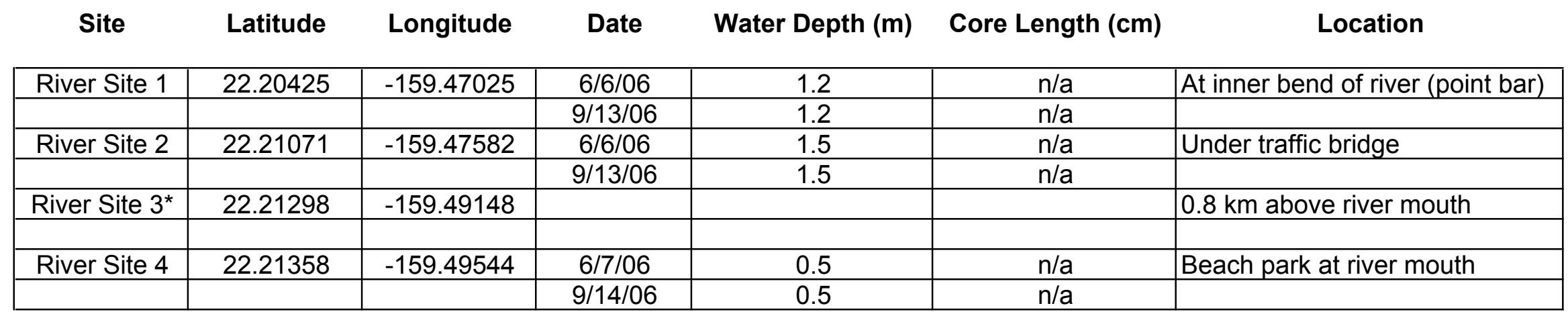

*River Site 3 was sampled in August 2005 (see Draut and others, 2006), but not sampled during 2006.

Table 4. Locations and details of river-bed samples collected in June and September 2006. 


\begin{tabular}{c|c|c|c|c|l|} 
Site & $\begin{array}{c}\text { Latitude } \\
\text { (WGS84) }\end{array}$ & $\begin{array}{c}\text { Longitude } \\
\text { (WGS84) }\end{array}$ & Date & Tocation \\
\hline M1 & 22.21047 & -159.47440 & $9 / 12 / 06$ & Taro field & Weir check gate taro fields, first ditch \\
\hline M2 & 22.21045 & -159.47437 & $9 / 12 / 06$ & Taro field & Plowed taro field \\
\hline M3 & & & $9 / 12 / 06$ & Riverbank & Eastern side of Hanalei River at USGS gaging station \\
\hline M4 & 22.17937 & -159.46602 & $9 / 12 / 06$ & Riverbank & $0-1 \mathrm{~cm}$ \\
\hline M5 & 22.17975 & -159.46620 & $9 / 12 / 06$ & Riverbank & $10 \mathrm{~m}$ North of gaging station \\
\hline M6 & 22.17975 & -159.46620 & $9 / 12 / 06$ & Riverbank & $105 \mathrm{~cm}$ from river, 120 from top of bank \\
\hline M7 & 22.17975 & -159.46620 & $9 / 12 / 06$ & Riverbank & 0-5 cm top of bank \\
\hline M8 & 22.18003 & -159.46650 & $9 / 12 / 06$ & Forest & About 50 m West of gaging station, in the forest \\
\hline & & & & & North side of highway opposite bamboo grove, 3 m from \\
M9 & 22.20887 & -159.48295 & $9 / 12 / 06$ & Grassland & edge of river in "thick" grass. \\
\hline M10 & 22.20840 & -159.47968 & $9 / 12 / 06$ & Taro field & Taro field \\
\hline M11 & 22.20880 & -159.47950 & $9 / 12 / 06$ & Taro field & Taro field, plowed \\
\hline M12 & 22.15960 & -159.45908 & $9 / 13 / 06$ & Dirt road & Lower cut bank just above road level 30 cm channel sample \\
\hline M13 & 22.16435 & -159.45878 & $9 / 13 / 06$ & Dirt road & Mud settled in low region of the road- right over culvert \\
\hline M14 & 22.15043 & -159.45865 & $9 / 13 / 06$ & Dirt road & 0-2 cm in road, 20 degree grade 14X18 cm $2 \mathrm{~cm}$ \\
\hline M15 & 22.20033 & -159.46803 & $9 / 13 / 06$ & Riverbank & Farthest upstream point reachable by kayak, on river bank \\
\hline M16 & 22.20887 & -159.48295 & $9 / 13 / 06$ & Riverbank & $0.8 \mathrm{~m}$ below the top of the bank \\
\hline
\end{tabular}

Table 5. Locations and details of upland samples collected in September 2006. 


\begin{tabular}{|c|c|c|c|c|c|c|c|c|}
\hline Site & Month & Core & $\begin{array}{c}{ }^{7} \mathrm{Be} \\
\left(\mathrm{dpm} / \mathrm{cm}^{2}\right)\end{array}$ & $\begin{array}{c}\text { Complete } \\
\text { inventory } \\
\text { recovered? }\end{array}$ & $\begin{array}{c}{ }^{137} \mathrm{Cs} \\
\left(\mathrm{dpm} / \mathrm{cm}^{2}\right)\end{array}$ & $\begin{array}{c}\text { Complete } \\
\text { inventory } \\
\text { recovered? }\end{array}$ & $\begin{array}{c}\text { Excess }{ }^{210} \mathrm{~Pb} \\
\left(\mathrm{dpm} / \mathrm{cm}^{2}\right) \\
\end{array}$ & $\begin{array}{l}\text { Complete } \\
\text { inventory } \\
\text { recovered? }\end{array}$ \\
\hline \multirow[t]{4}{*}{ A } & Jun-05 & K0605-1 & 9.86 & yes & 2.53 & yes & 220.00 & yes, $56 \mathrm{~cm}$ \\
\hline & Aug-05 & K0805-7 & 0.07 & yes & 1.77 & yes & 219.32 & yes, $40 \mathrm{~cm}$ \\
\hline & Jun-06 & K0606-A & 55.89 & yes & 6.40 & yes & 286.83 & yes \\
\hline & Sep-06 & K0906-A & 18.27 & yes & 7.29 & yes & 304.13 & no \\
\hline \multirow[t]{4}{*}{ B } & Jun-05 & K0605-2 & 0.00 & yes & 0.34 & yes & 251.68 & no, but almost \\
\hline & Aug-05 & K0805-1 & 0.59 & yes & 2.52 & probably & 272.34 & no, but almost \\
\hline & Jun-06 & K0606-B & 2.38 & $\mathrm{no}, 8 \mathrm{~cm}$ & 0.00 & $\mathrm{no}, 8 \mathrm{~cm}$ & 85.34 & $\mathrm{no}, 8 \mathrm{~cm}$ \\
\hline & Sep-06 & K0906-B & 0.70 & $\mathrm{no}, 4 \mathrm{~cm}$ & 0.01 & yes, $4 \mathrm{~cm}$ & 47.58 & no \\
\hline \multirow[t]{4}{*}{ C } & Jun-05 & K0605-3 & 0.09 & yes & 0.10 & yes & 304.43 & no, but almost \\
\hline & Aug-05 & K0805-3 & 0.00 & yes & 0.03 & yes & 53.58 & no, $20 \mathrm{~cm}$ \\
\hline & Jun-06 & K0606-C & 8.06 & yes & 9.87 & yes & 438.37 & $\mathrm{no}, 47 \mathrm{~cm}$ \\
\hline & Sep-06 & K0906-C & 0.84 & possibly & 1.16 & possibly & 274.31 & no \\
\hline \multirow[t]{4}{*}{ D } & Jun-05 & K0605-4 & 0.05 & yes & 0.02 & yes & 108.39 & no, $20 \mathrm{~cm}$ \\
\hline & Aug-05 & K0805-4 & 0.34 & yes & 0.04 & yes & 135.34 & $\mathrm{no}, 30 \mathrm{~cm}$ \\
\hline & Jun-06 & K0606-D & 2.31 & $\mathrm{no}, 10 \mathrm{~cm}$ & 0.00 & no, $10 \mathrm{~cm}$ & 77.67 & $\mathrm{no}, 10 \mathrm{~cm}$ \\
\hline & Sep-06 & K0906-D & 0.00 & yes & 0.03 & yes & 43.16 & no \\
\hline \multirow[t]{4}{*}{$\mathbf{E}$} & Jun-05 & K0605-5 & 0.34 & yes & 0.01 & yes & 167.23 & $\mathrm{no}, 20 \mathrm{~cm}$ \\
\hline & Aug-05 & K0805-6 & 0.00 & yes & 0.00 & yes & 172.58 & $\mathrm{n}, 18 \mathrm{~cm}$ \\
\hline & Jun-06 & K0606-E & 2.33 & $\mathrm{no}, 10 \mathrm{~cm}$ & 0.13 & no, $10 \mathrm{~cm}$ & 110.01 & $\mathrm{n}, 10 \mathrm{~cm}$ \\
\hline & Sep-06 & K0906-E & 3.46 & yes & 0.83 & no & 192.56 & no \\
\hline \multirow[t]{4}{*}{$\mathbf{F}$} & Jun-05 & K0605-6 & 0.31 & no & 0.52 & no & 28.37 & $\mathrm{no}, 4 \mathrm{~cm}$ \\
\hline & Aug-05 & K0805-2 & 0.81 & yes & 1.42 & yes & 133.53 & yes, $40 \mathrm{~cm}$ \\
\hline & Jun-06 & K0606-F & 90.75 & yes & 11.29 & yes & 380.90 & yes \\
\hline & Sep-06 & K0906-F & 21.01 & yes & 6.29 & yes & 319.20 & no \\
\hline \multirow[t]{4}{*}{ G } & Jun-05 & K0605-7 & 1.56 & yes & 1.45 & $\mathrm{no}, 15 \mathrm{~cm}$ & 57.30 & $\mathrm{no}, 15 \mathrm{~cm}$ \\
\hline & Aug-05 & K0805-5 & 2.25 & no? $18 \mathrm{~cm}$ & 2.65 & no & 90.74 & $\mathrm{no}, 18 \mathrm{~cm}$ \\
\hline & Jun-06 & K0606-G & 7.41 & yes & 7.27 & yes & 196.29 & $\mathrm{no}, 40 \mathrm{~cm}$ \\
\hline & Sep-06 & K0906-G & 1.84 & yes & 1.18 & yes & 85.83 & $\mathrm{no}, 12 \mathrm{~cm}$ \\
\hline \multirow[t]{2}{*}{$\mathbf{H}$} & Jun-06 & $\mathrm{K} 0606-\mathrm{H}$ & 2.55 & $\mathrm{no}, 5 \mathrm{~cm}$ & 0.05 & $\mathrm{no}, 5 \mathrm{~cm}$ & 36.00 & $\mathrm{no}, 5 \mathrm{~cm}$ \\
\hline & Sep-06 & K0906-H & 3.95 & no & 0.45 & no & 92.08 & no \\
\hline \multirow[t]{2}{*}{ I } & Jun-06 & K0606-I & 102.42 & yes & 13.43 & yes & 534.16 & possibly \\
\hline & Sep-06 & K0906-I & 75.75 & yes & 41.65 & $\mathrm{no}, 53 \mathrm{~cm}$ & 1134.54 & $\mathrm{no}, 53 \mathrm{~cm}$ \\
\hline
\end{tabular}

Table 6. Inventory of ${ }^{7} \mathrm{Be},{ }^{137} \mathrm{Cs}$, and excess ${ }^{210} \mathrm{~Pb}$ in the sediment at all core sites. Inventories were calculated from gamma-counted activity. Units are disintegrations per minute (dpm) per $\mathrm{cm}^{2}$ of sea-floor surface area.

Data from June and August 2005 (Draut and others, 2006) are shown for comparison with the data from June and September 2006. Values in bold text indicate the sample with the highest inventory of ${ }^{7} \mathrm{Be}$ at each core site. 
Approximately $25 \mathrm{~g}$ of each sample were soaked in hydrogen peroxide to remove organic material and washed via centrifugation. Samples were wet-sieved using a 2-mm sieve to collect the gravel, a $63-\mu$ sieve to collect the sand, and a 1,000-ml graduated cylinder to collect silt and clay. The gravel and sand fractions were dried and weighed to determine the percent, by mass, of the sample. Silt and clay percentages were determined by pipette analysis based on Stokes' law. A representative sub-sample was taken from each bulk sediment sample for percent carbonate determination. Total inorganic carbon and percent carbonate were analyzed using a UIC CM5012 $\mathrm{CO}_{2}$ coulometer and a CM5130 acidification module following UIC standard procedures.

\section{Magnetic-Property Analyses}

Magnetic properties of sediment samples from cores at Sites A, C, and I, as well as from upland sites in the drainage basin (24 samples) and selected samples of suspended sediment captured in sediment traps within Hanalei Bay, were analyzed at the USGS laboratory in Denver, Colo. Dried sediment samples packed into $3.2-\mathrm{cm}^{3}$ plastic cubes and normalized for sample mass were measured. In this study, remanent magnetization was measured using a $90-\mathrm{Hz}$ spinner magnetometer with a sensitivity of $\sim 10^{-5} \mathrm{Am}^{-1}$. ARM was imparted in a DC induction of $0.1 \mathrm{mT}$ in the presence of a decaying, alternating induction from 100 to $0 \mathrm{mT}$. Magnetic susceptibility was measured at frequencies of $600 \mathrm{~Hz}$ (MSlf) and 6,000 Hz (MShf) and frequency-dependent MS (FDMS; [[MSlf-MShf]/MSlf]) was calculated as a percentage.

\section{Results}

Figure 3 shows water and sediment discharge for the Hanalei River during the study interval, and for the three most recent complete water years (2004-2006), along with rainfall measured at two rain gages in the Hanalei watershed (http://hi.water.usgs.gov/). Four intervals of sampling during this study are shown in figure 3a; the first two intervals, completed in June and August 2005, were discussed by Draut and others (2006), whereas the latter two intervals (June and September 2006) are the focus of this report. During this two-year study, the largest flood events occurred between February and April 2006, when the Hawaiian Islands experienced sustained heavy rainfall causing abundant terrestrial runoff to the coastal ocean. Peak flood magnitude during that interval occurred on February 21, 2006, when daily mean water discharge was $119 \mathrm{~m}^{3} \mathrm{~s}^{-1}$. On that one day, the fluvial suspended-sediment load (12,700 metric tons) accounted for nearly a third of the total sediment carried by the Hanalei River between September 2005 and June 2006. After the winter and spring 2006 floods subsided, the river was relatively quiescent until a flood of $46 \mathrm{~m}^{3}$ $\mathrm{s}^{-1}$ occurred on August 7, 2006-smaller than floods the previous winter, but still a substantial discharge event for the summer season (fig. 3).

Locations and details of collection for each core, seabed, sediment-trap, river-bed, and upland sample are listed in tables $1-5$. Inventories of ${ }^{7} \mathrm{Be},{ }^{137} \mathrm{Cs}$, and excess ${ }^{210} \mathrm{~Pb}$ for each core site are reported in table 6 along with similar data from June and August 2005 for comparison (see Draut and others, 2006). Complete descriptions of sediment from all 18 cores (nine each in June and September 2006) are listed in appendices 1 and 2. Appendix 3 includes results of all analyses conducted on all sediment samples collected in 2005 and 2006 as part of this project.

Figure 4 shows the proportions of mud in seabed samples in Hanalei Bay in June and September 2006. Thirteen seabed samples collected in June showed mud percentages that ranged from 0.02-94.4 percent (highest at the northern end of the Black Hole; table 2, fig. 4A), with a mean of 11.6 percent, a median of 2.36 percent, and a standard deviation among the thirteen samples of 25.5 percent. Fourteen seabed samples collected in September 2006 showed mud 
Figure 3. Water and sediment discharge from the Hanalei River, and rainfall in the Hanalei watershed, during water years 2004-2006 (October 1, 2004, through September 30, 2006). Data were measured at USGS gaging station 16103000 ("Hanalei River near Hanalei"), located $8.2 \mathrm{~km}$ upstream of the river mouth) and were provided by the USGS Pacific Islands Water Science Center in Honolulu, Hawai'i (http://hi.water.usgs.gov/). (A). Water discharge; red vertical lines indicate times of sediment-core collection in Hanalei Bay. (B) Rainfall measured at two USGS rain gages in the Hanalei watershed; gage 220427159300201 is in the mountains near the headwaters of the river, and gage 221101159280801 is near the town of Hanalei on the lower floodplain. (C). Suspended-sediment concentration ( $\mathrm{mg} \mathrm{L}^{-1}$ ) measured at gaging station 16103000. (D). Fluvial sediment load (in metric tons per day) based on a rating curve and suspendedsediment samples analyzed by the Water Resources Division of the USGS. 

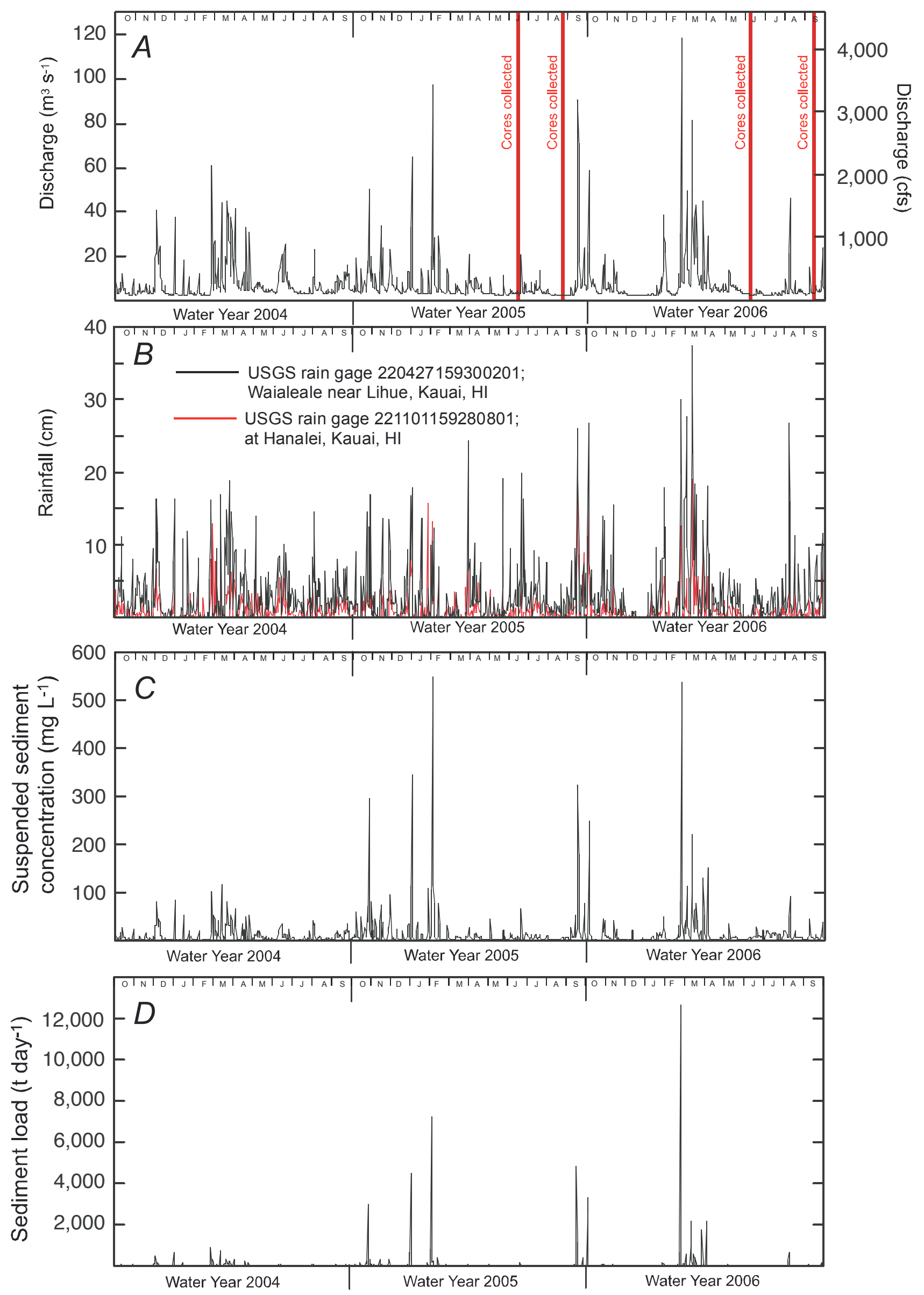

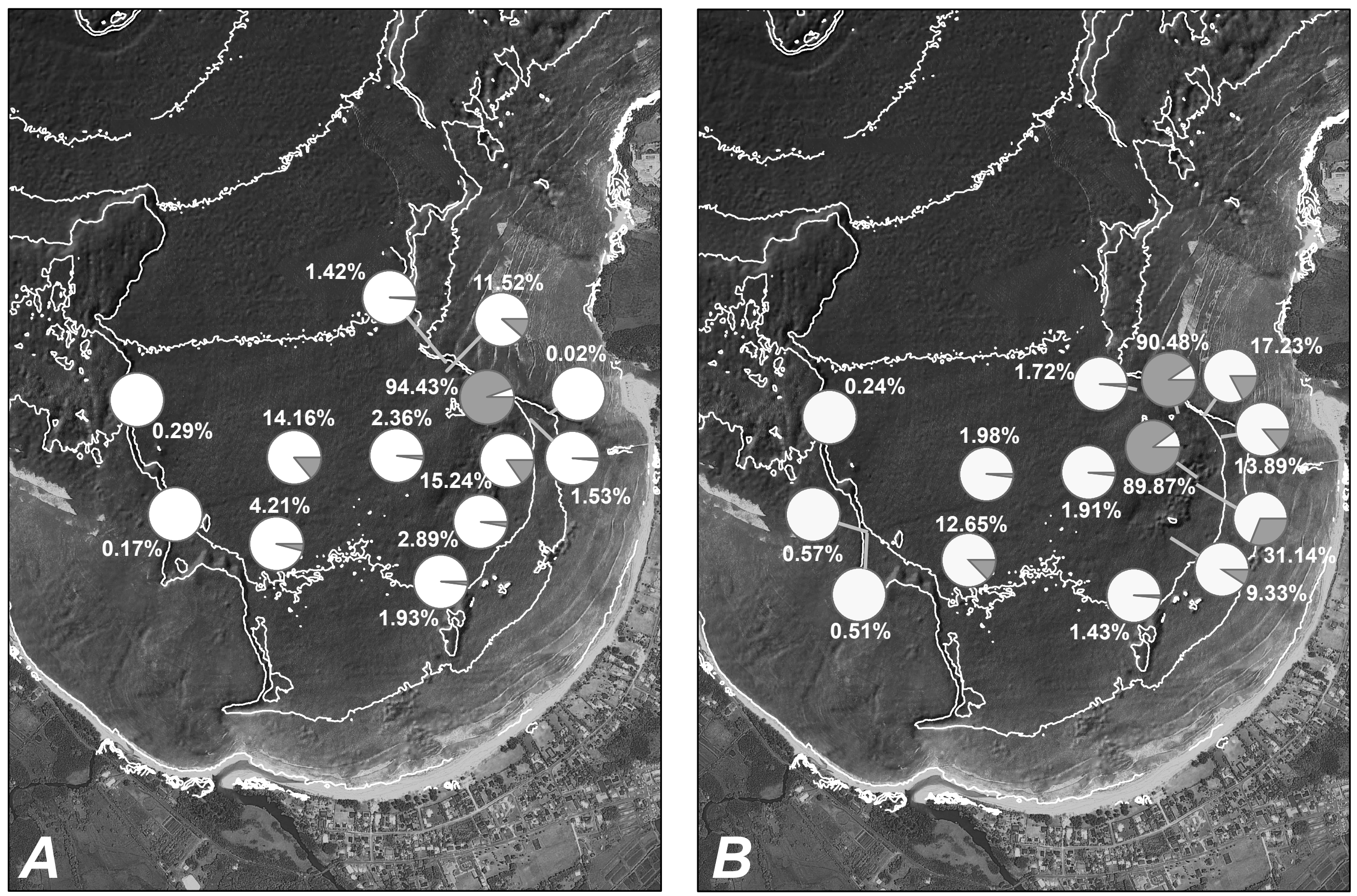

Figure 4. Proportion of mud in seabed grab samples collected in (A) June and (B) September, 2006. Numbers next to pie charts represent the percentage dry mass of sediment finer than 63 microns at each location. 
percentages ranging from 0.24-90.5 percent (highest at the northern end of the Black Hole; table 2, fig. $4 B$ ), with a mean of 19.5 percent, a median of 5.66 percent, and a standard deviation among the fourteen samples of 31.2 percent.

Figures 5 to 22, ordered by core site name (locations shown in fig. $2 A$ ), show activity profiles for ${ }^{7} \mathrm{Be},{ }^{137} \mathrm{Cs}$, and excess ${ }^{210} \mathrm{~Pb}$ in the $18 \mathrm{cores}$, and sedimentary-facies diagrams. Isotope activities have been decay-corrected to the time of sample collection, and are reported as disintegrations per minute per dry gram of salt-free sediment. Magnetic properties of the cores in which these were analyzed are illustrated in depth profiles in figures 23 to 28. Facies showed substantial spatial heterogeneity within the bay; examples of different sediment types observed are shown in figure 29.

Cores collected in the Black Hole (at Sites A, F, and I; figs. 5, 6, 15, 16, 21, and 22) contained unconsolidated very fine-grained sediment and organic debris, including wood and leaf fragments in the upper portions of the cores (commonly the upper $\sim 10 \mathrm{~cm}$ ) underlain by horizons containing both carbonate sand and dark mud (with the exception of Site I in September 2006, which contained essentially only dark mud; fig. 22). One siliciclastic pebble was found at Site A in September (fig. 6). In June, all three Black Hole core sites showed substantial ${ }^{7} \mathrm{Be}\left(>10 \mathrm{dpm} \mathrm{g}^{-1}\right)$ and ${ }^{137} \mathrm{Cs}\left(\sim 1 \mathrm{dpm} \mathrm{g} \mathrm{g}^{-1}\right)$ activity and excess ${ }^{210} \mathrm{~Pb}\left(20-40 \mathrm{dpm} \mathrm{g} \mathrm{g}^{-1}\right)$ down to depths of 10-20 cm beneath the sea floor. By September $2006,{ }^{7}$ Be activity had decreased at the three Black Hole sites to $5 \mathrm{dpm} \mathrm{g}{ }^{-1}$ or less, while ${ }^{137} \mathrm{Cs}$ and excess ${ }^{210} \mathrm{~Pb}$ were comparable to June values.

The cores from Site G (figs. 17 and 18), on the eastern side of the bay near the river mouth, contained a higher proportion of carbonate sand relative to fine dark mud compared with the Black Hole cores. In June and September 2006, sediment near the top of cores from Site $\mathrm{G}$ contained ${ }^{7} \mathrm{Be}$, ${ }^{137} \mathrm{Cs}$, and excess ${ }^{210} \mathrm{~Pb}$, though at activity levels almost an order of magnitude lower than levels sampled at the same time at Black Hole Sites A, F, and I. Plant debris was present from depths of $\sim 12-30 \mathrm{~cm}$ at Site $\mathrm{G}$ in June 2006, and near $40 \mathrm{~cm}$ in September.

Site $\mathrm{C}$, located along the coral wall on the northeastern side of the bay, contained two dark mud horizons in June 2006 (fig. 9), spanning depths of 0-2 cm and 28-34 cm below the sea floor, respectively. The mud horizons contained elevated ${ }^{7} \mathrm{Be}$ and ${ }^{137} \mathrm{Cs}$, although at lower activity levels than in the Black Hole $\left({ }^{7} \mathrm{Be}\right.$ at $\sim 4 \mathrm{dpm} \mathrm{g}^{-1}$ in surface sediment at Site C compared with $\sim 15 \mathrm{dpm} \mathrm{g}^{-1}$ in surface sediment of Site A in June 2006). As of September 2006, the upper mud layer was no longer evident at Site C; the September core contained dark mud between 46 and $49 \mathrm{~cm}$ deep but was otherwise dominated by carbonate sand (fig. 10).

Sediment in cores from Sites B, D, E, and H, all located farther from the Hanalei River mouth than the aforementioned sites and outside of the Black Hole, were dominated by carbonate sand in June and September 2006 (figs. 7, 8, 11, 12, 13, 14, 19, and 20). A thin (mm-scale) surface "fluff" layer of mobile fine sediment and organic matter was observed on occasion at these sites and at other locations in the middle and western part of Hanalei Bay (Draut and others, 2006) but carbonate sand was much more prevalent than dark mud in cores. Sites B, D, E, and H contained ${ }^{7}$ Be activity typically $<1 \mathrm{dpm} \mathrm{g}^{-1},{ }^{137} \mathrm{Cs}<0.5 \mathrm{dpm} \mathrm{g}^{-1}$, and excess ${ }^{210} \mathrm{~Pb}<10 \mathrm{dpm} \mathrm{g}$.

In general, samples from sediment traps at sites in the bay that were nearer to the Hanalei River mouth contained substantially higher levels of ${ }^{7} \mathrm{Be}$ and ${ }^{137} \mathrm{Cs}$ than sediment collected at traps farther from the river mouth. The tube trap deployed at the Inner and Outer Wall sites in 2006 (locations shown in fig. $2 A$ ) collected sediment with ${ }^{7} \mathrm{Be}$ ranging from $6.5-10.5 \mathrm{dpm} \mathrm{g}{ }^{-1},{ }^{137} \mathrm{Cs}$ from $0.7-1 \mathrm{dpm} \mathrm{g}^{-1}$, and excess ${ }^{210} \mathrm{~Pb}$ ranging from $18-35 \mathrm{dpm} \mathrm{g}^{-1}$. Samples from the South Central tube and rotating sediment traps displayed variable activities of all three isotopes, with ${ }^{7} \mathrm{Be}$ ranging from 0-35 dpm $\mathrm{g}^{-1},{ }^{137} \mathrm{Cs}$ from $0-0.87 \mathrm{dpm} \mathrm{g}^{-1}$, and excess ${ }^{210} \mathrm{~Pb}$ ranging from $\sim 24-123 \mathrm{dpm} \mathrm{g}^{-1}$. The 


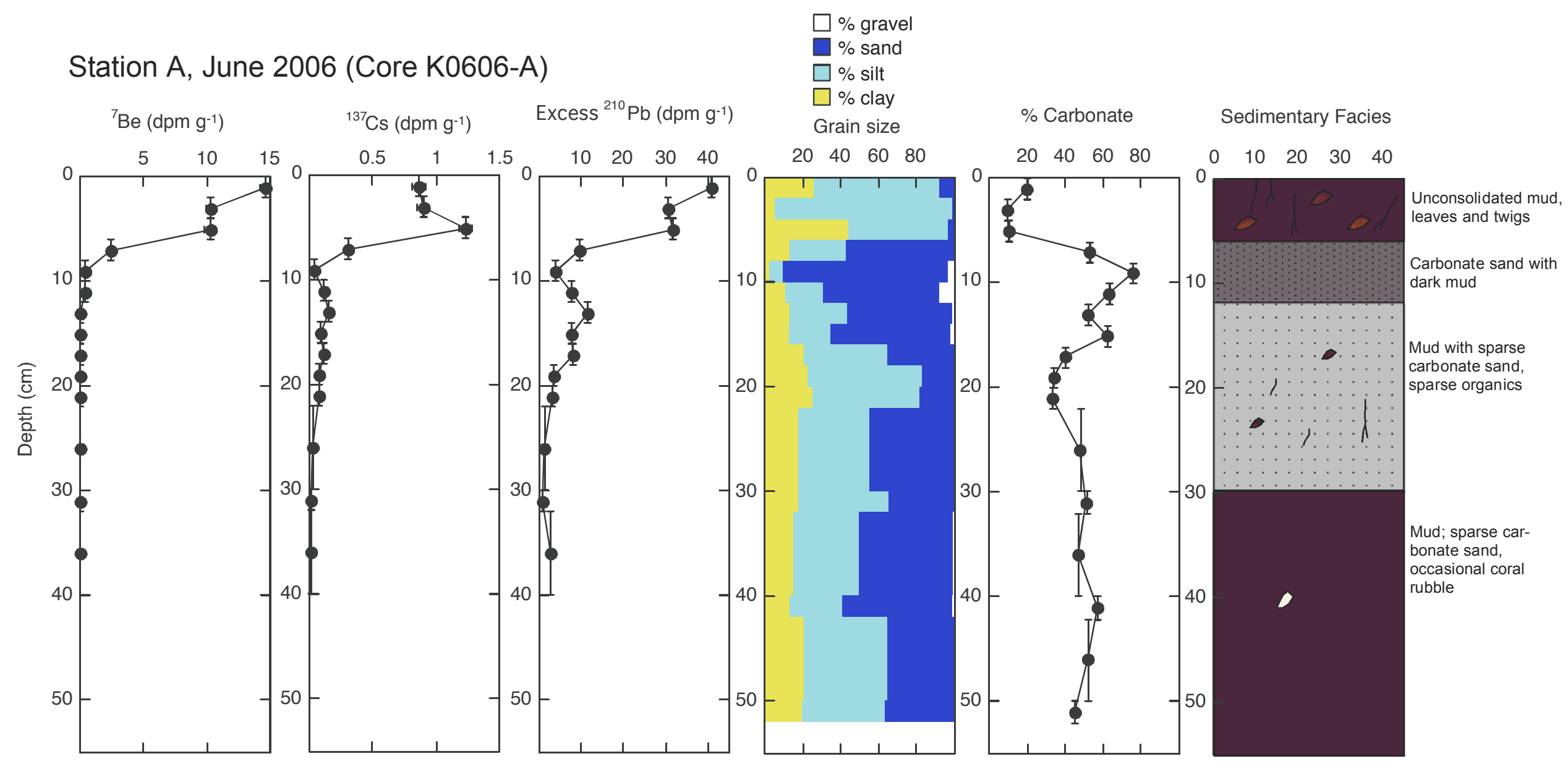

Figure 5. Sedimentary facies and isotope activity at Station A in June 2006 (Core K0606-A). Activities of 7 Be, 137 Cs, and excess 210Pb, all decay-corrected to the date of sample collection, are shown in disintegrations per minute (dpm) per dry gram of salt-free sediment. This site is in the Black Hole, on the eastern side of Hanalei Bay, near the mouth of the Hanalei River. 


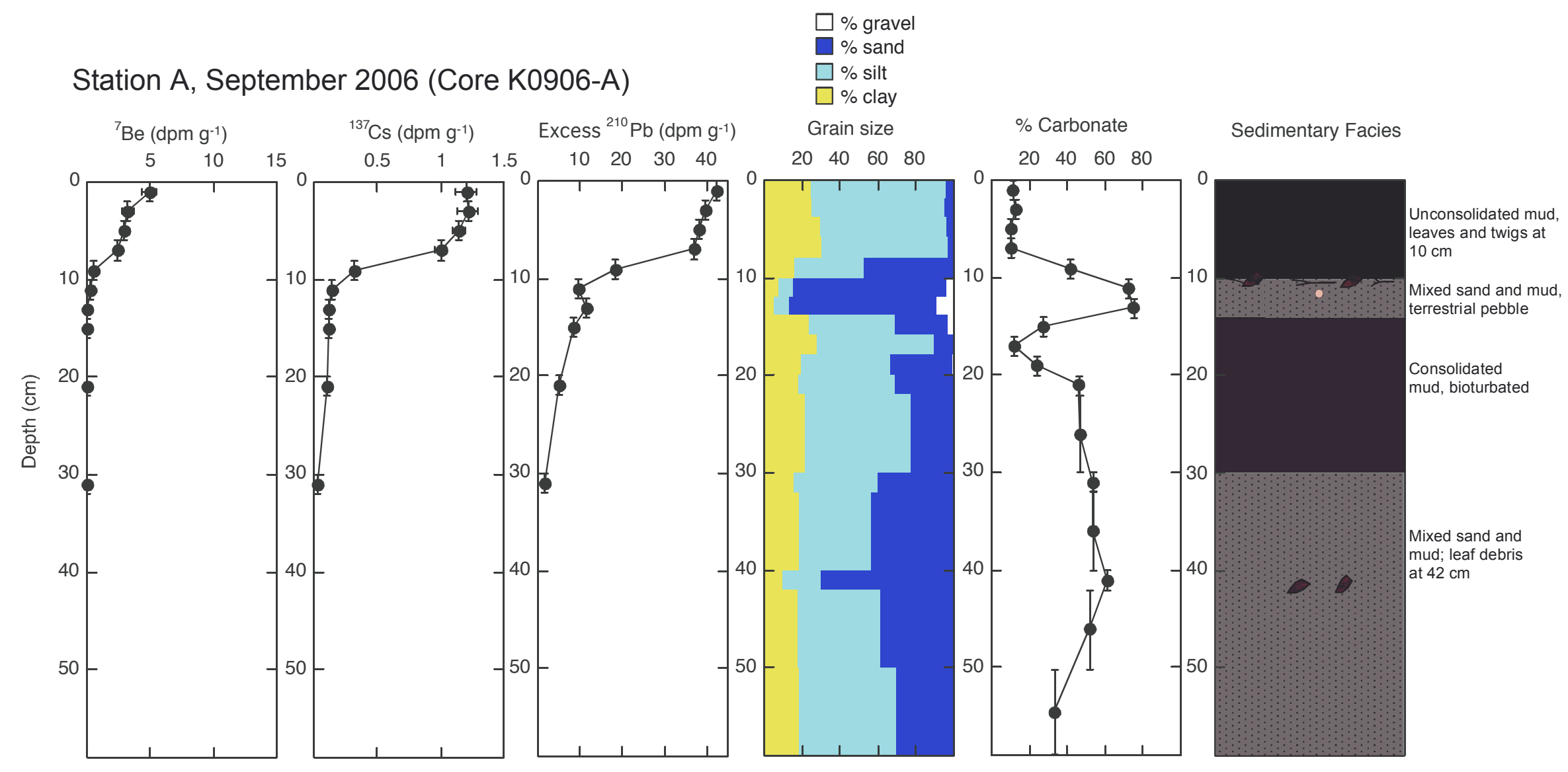

Figure 6. Sedimentary facies and isotope activity at Station A in September 2006 (Core K0906-A). Activities of 7Be, 137Cs, and excess 210Pb, all decay-corrected to the date of sample collection, are shown in disintegrations per minute (dpm) per dry gram of salt-free sediment. This site is in the Black Hole, on the eastern side of Hanalei Bay, near the mouth of the Hanalei River. 


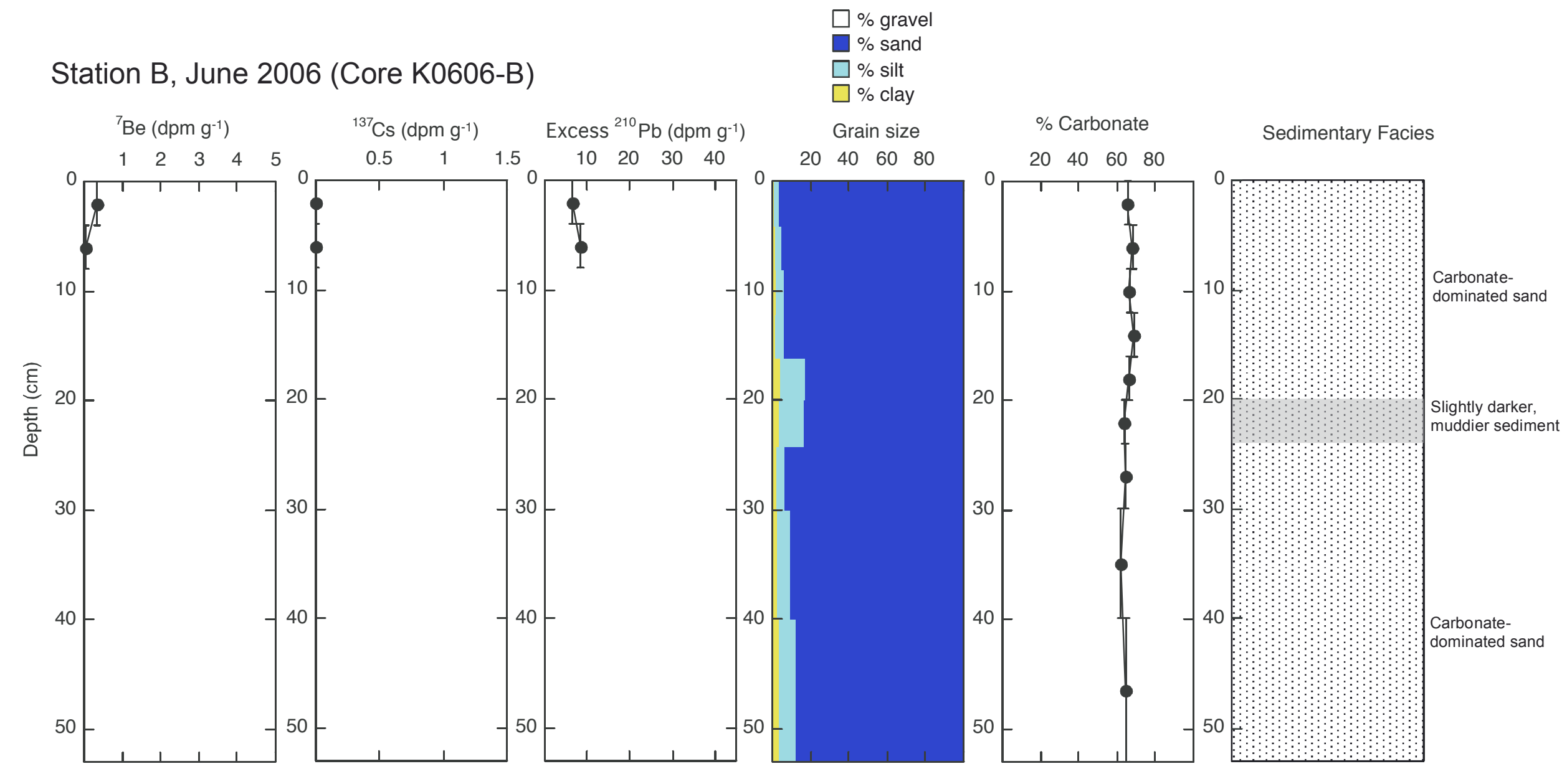

Figure 7. Sedimentary facies and isotope activity at Station B in June 2006 (Core K0606-B). Activities of 7Be, 137 Cs, and excess 210Pb, all decay-corrected to the date of sample collection, are shown in disintegrations per minute (dpm) per dry gram of salt-free sediment. This site is in the south-central part of Hanalei Bay. 


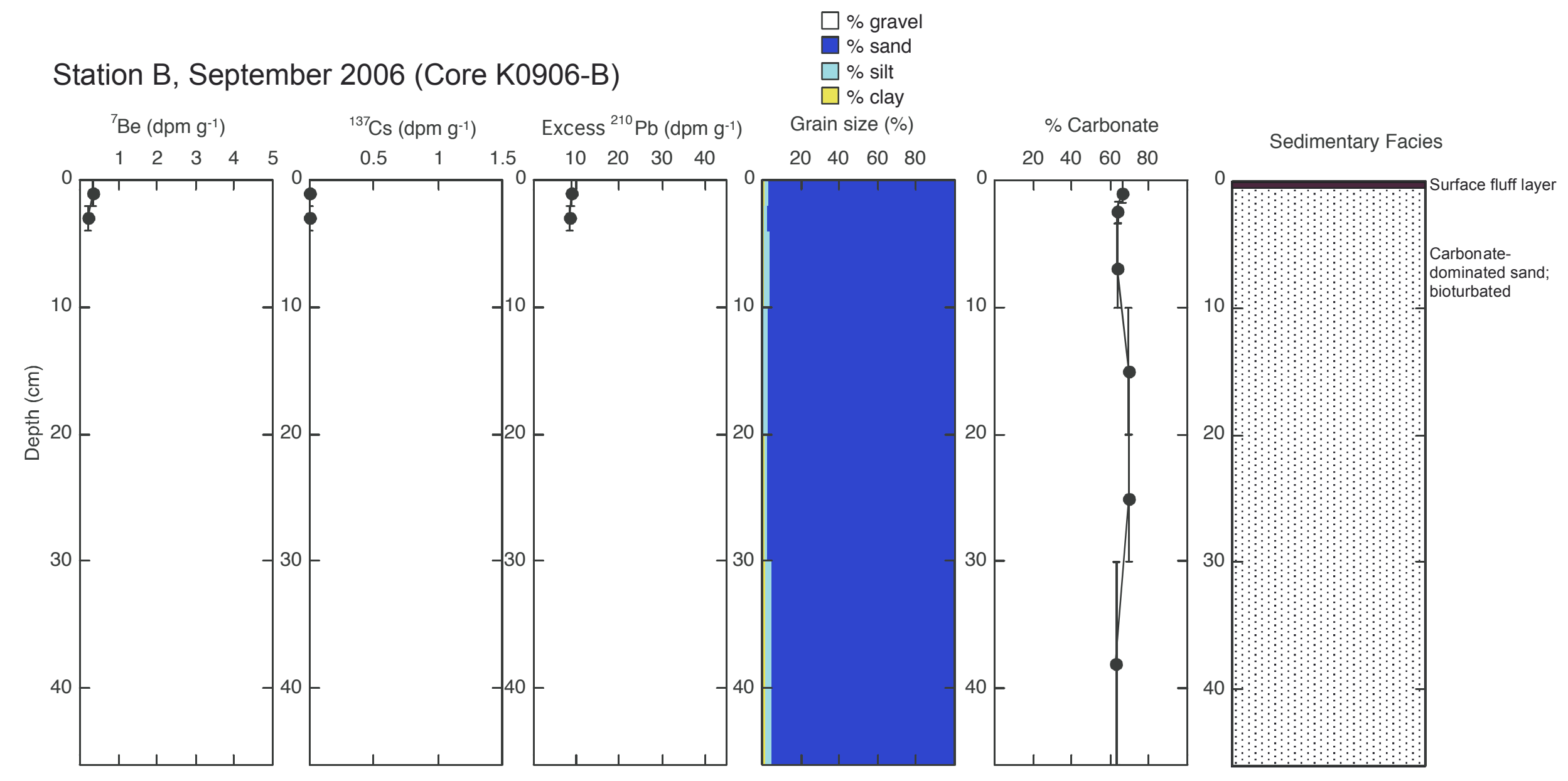

Figure 8. Sedimentary facies and isotope activity at Station B in September 2006 (Core K0906-B). Activities of 7Be, 137Cs, and excess 210Pb, all decaycorrected to the date of sample collection, are shown in disintegrations per minute (dpm) per dry gram of salt-free sediment. This site is in the southcentral part of Hanalei Bay. 
Station C, June 2006 (Core K0606-C)

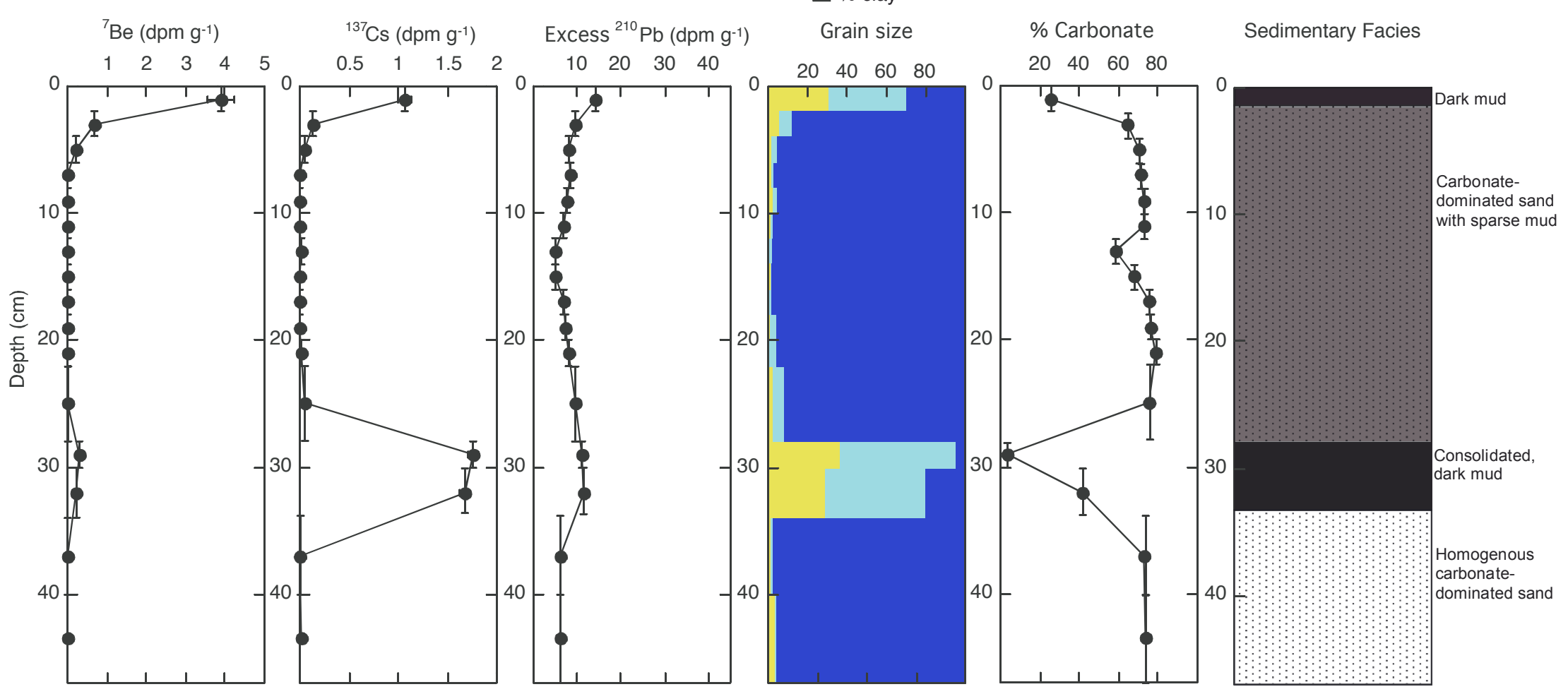

Figure 9. Sedimentary facies and isotope activity at Station C in June, 2006 (Core K0606-C). Activities of 7 Be, 137 Cs, and excess 210Pb, all decay-corrected to the date of sample collection, are shown in disintegrations per minute $(d p m)$ per dry gram of salt-free sediment. This site is on the northeastern side of Hanalei Bay, 8 $m$ away from the coral wall near the river mouth. 


\section{Station C, September 2006 (Core K0906-C) $\%$ sand}

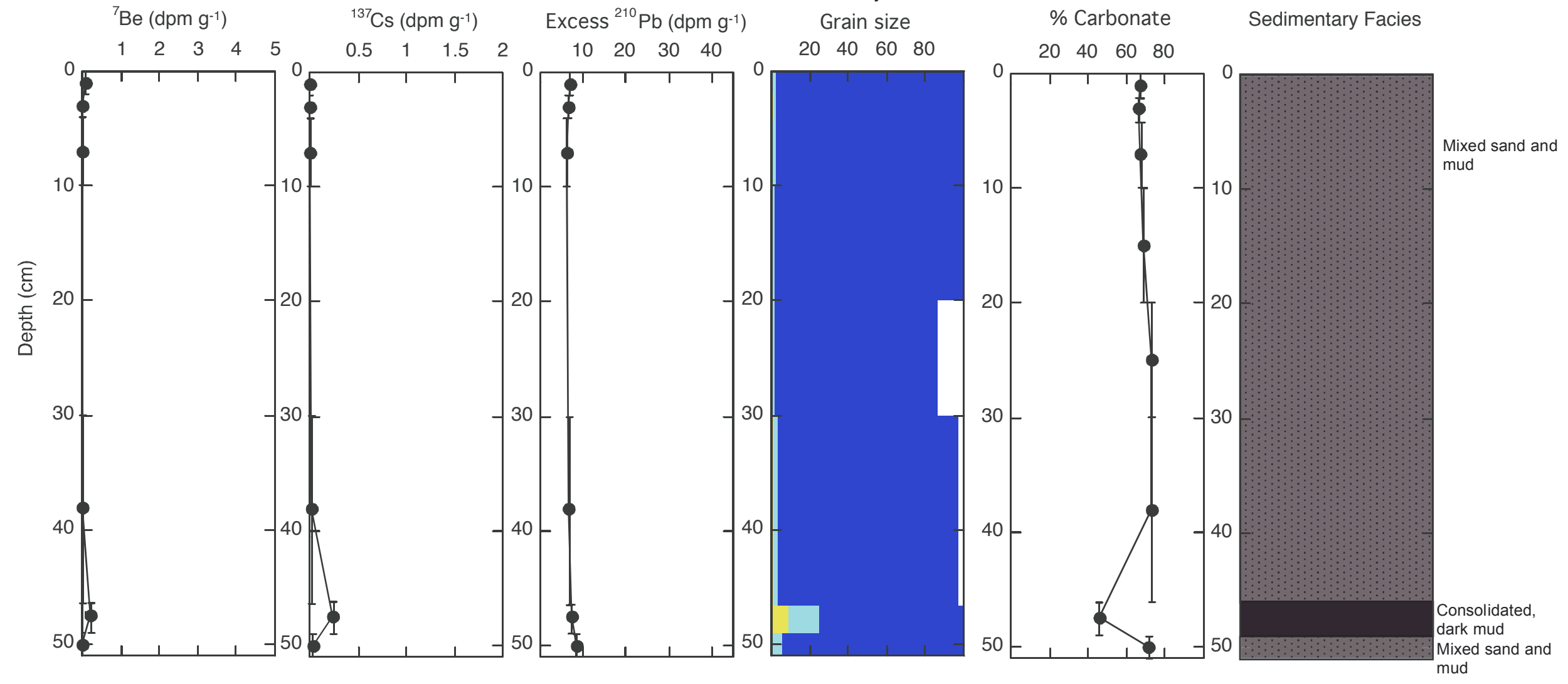

Figure 10. Sedimentary facies and isotope activity at Station C in September 2006 (Core K0906-C). Activities of 7Be, 137Cs, and excess 210Pb, all decay-corrected to the date of sample collection, are shown in disintegrations per minute (dpm) per dry gram of salt-free sediment. This site is on the northeastern side of Hanalei Bay, $\sim 8 \mathrm{~m}$ away from the coral wall near the river mouth. 


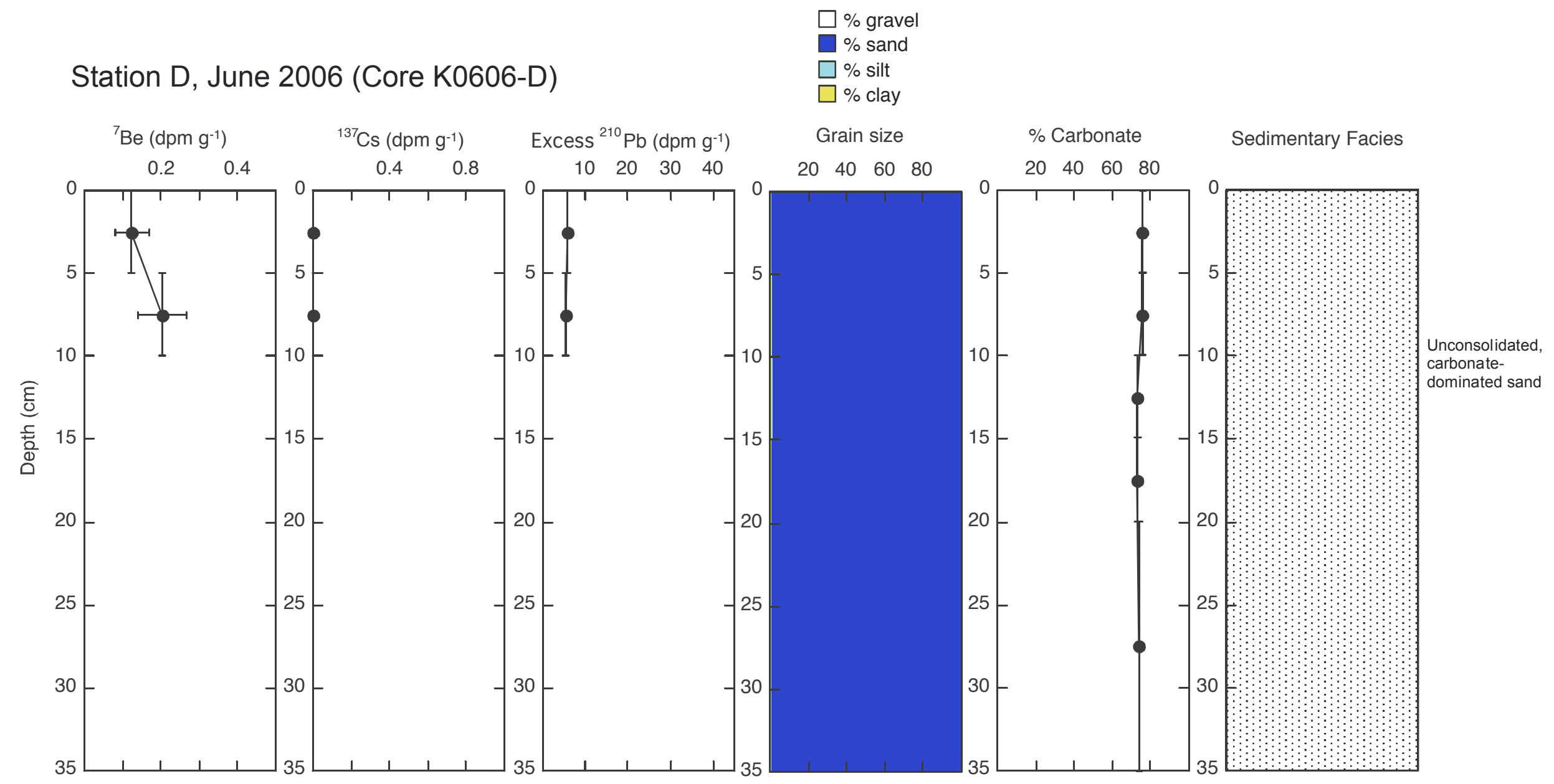

Figure 11. Sedimentary facies and isotope activity at Station D in June 2006 (Core K0606-D). Activities of 7Be, 137Cs, and excess 210Pb, all decaycorrected to the date of sample collection, are shown in disintegrations per minute $(\mathrm{dpm})$ per dry gram of salt-free sediment. This site is on the northwestern side of Hanalei Bay, $5 \mathrm{~m}$ away from a coral wall near the CRAMP site (in fig. 2A). 


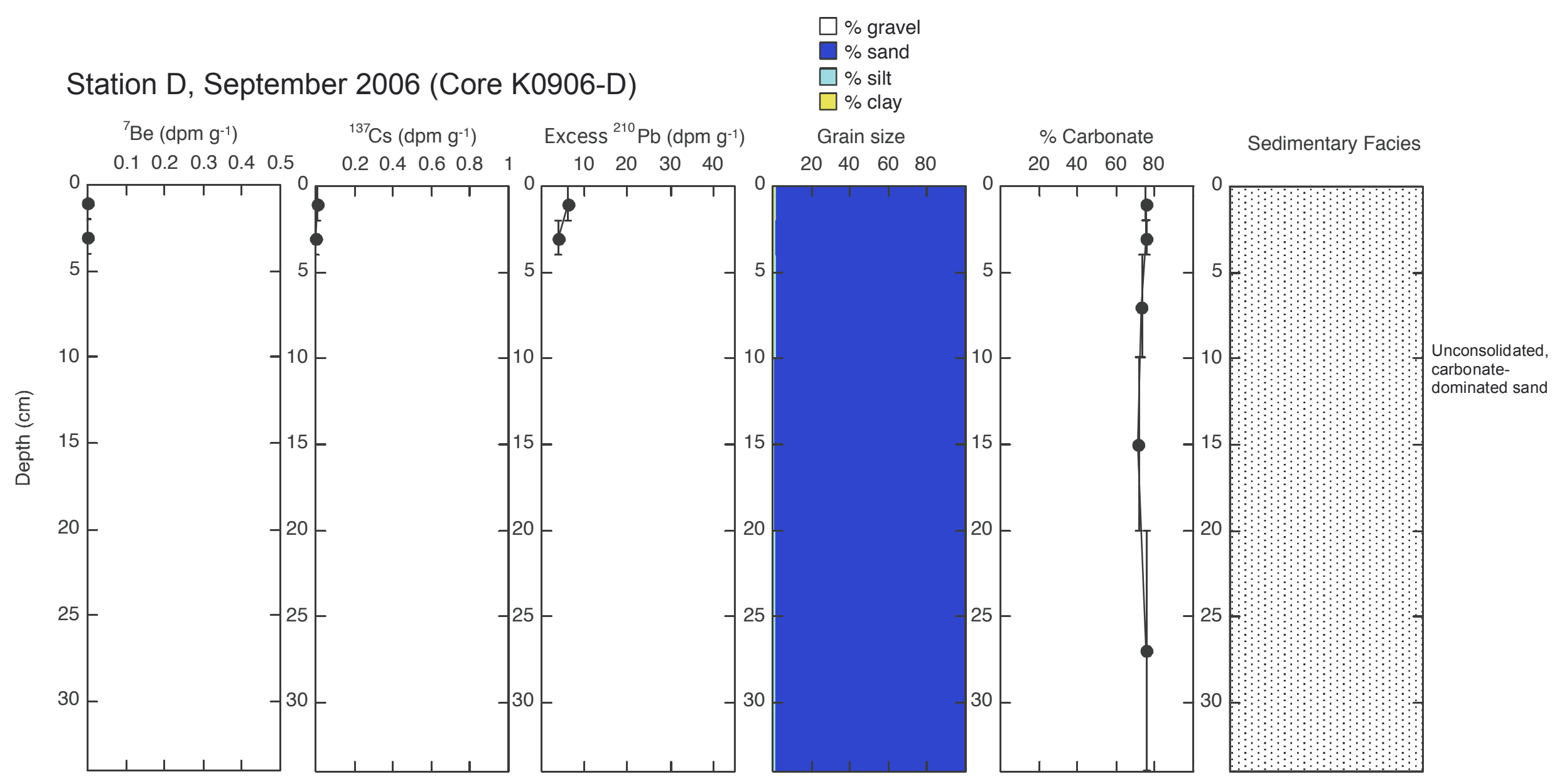

Figure 12. Sedimentary facies and isotope activity at Station D in September 2006 (Core K0906-D). Activities of 7Be, 137Cs, and excess 210Pb, all decaycorrected to the date of sample collection, are shown in disintegrations per minute (dpm) per dry gram of salt-free sediment. This site is on the northwestern side of Hanalei Bay, $\sim 5$ away from a coral wall near the CRAMP site (in fig. 2A). 


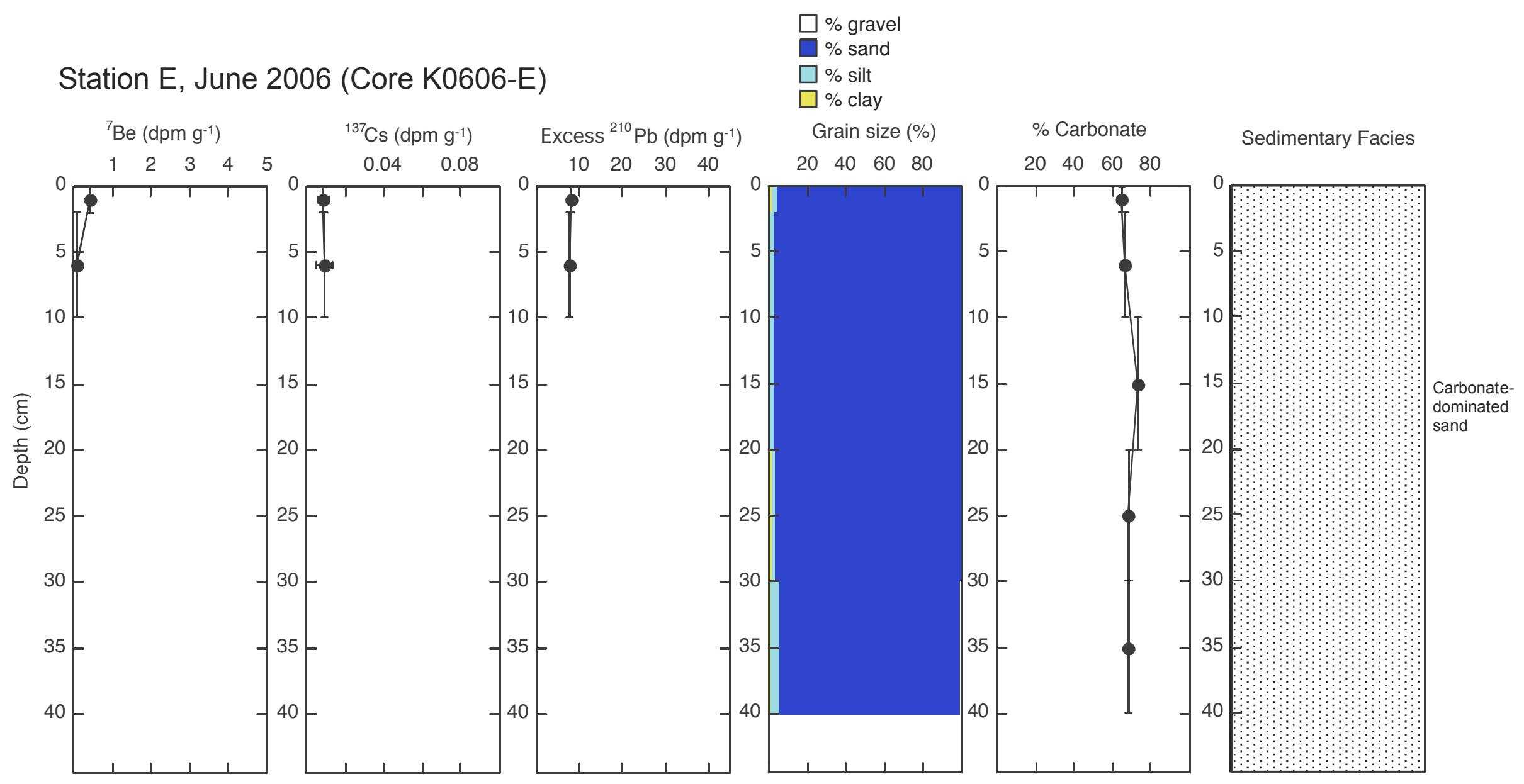

Figure 13. Sedimentary facies and isotope activity at Station E in June 2006 (Core K0606-E). Activities of 7Be, 137Cs, and excess 210Pb, all decay-corrected to the date of sample collection, are shown in disintegrations per minute (dpm) per dry gram of salt-free sediment. This site is in the middle of Hanalei Bay. 


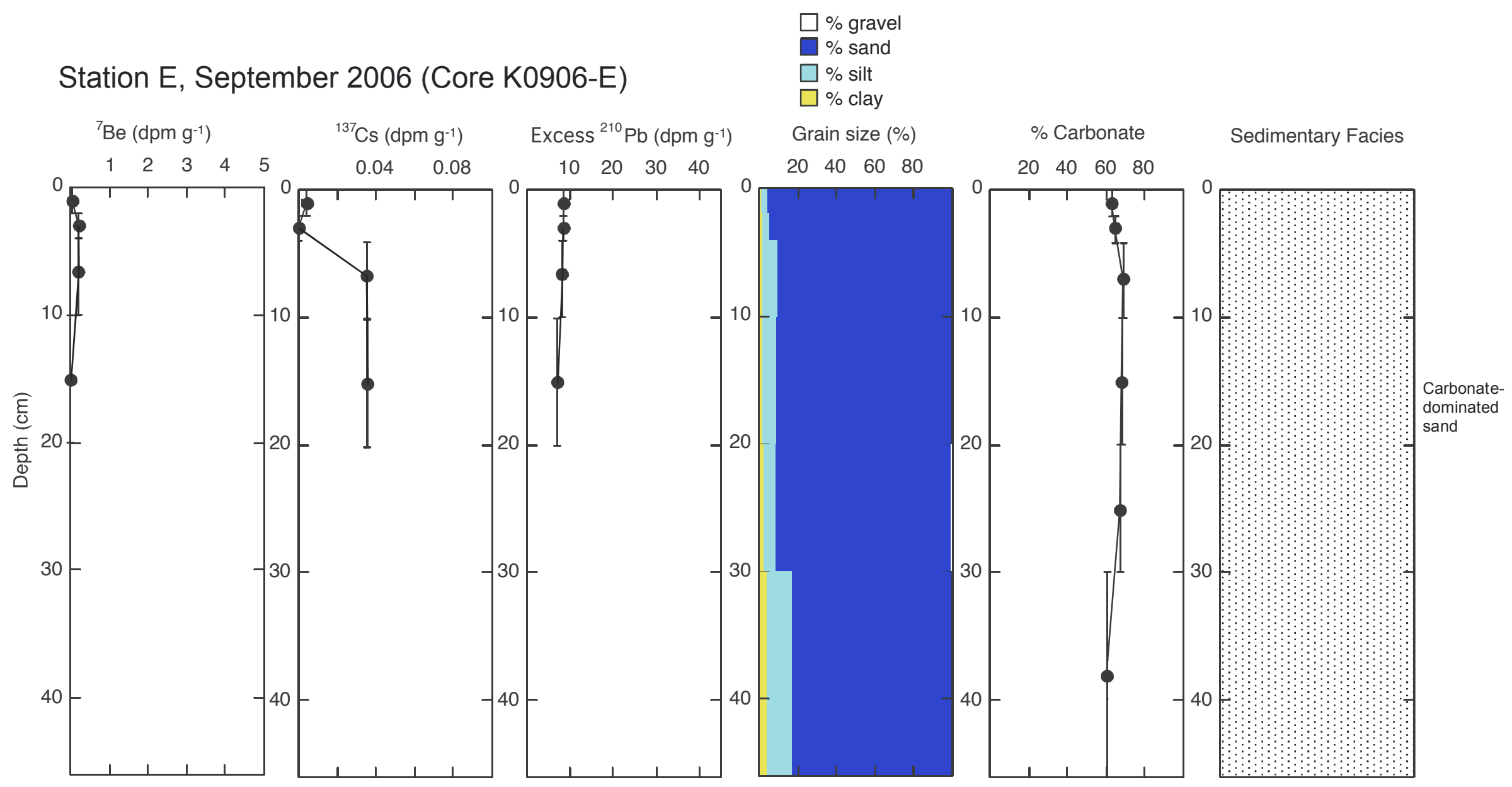

Figure 14. Sedimentary facies and isotope activity at Station E in September 2006 (Core K0906-E). Activities of 7Be, 137Cs, and excess 210Pb, all decay-corrected to the date of sample collection, are shown in disintegrations per minute (dpm) per dry gram of salt-free sediment. This site is in the middle of Hanalei Bay. 


\section{Station F, June 2006 (Core K0606-F)}

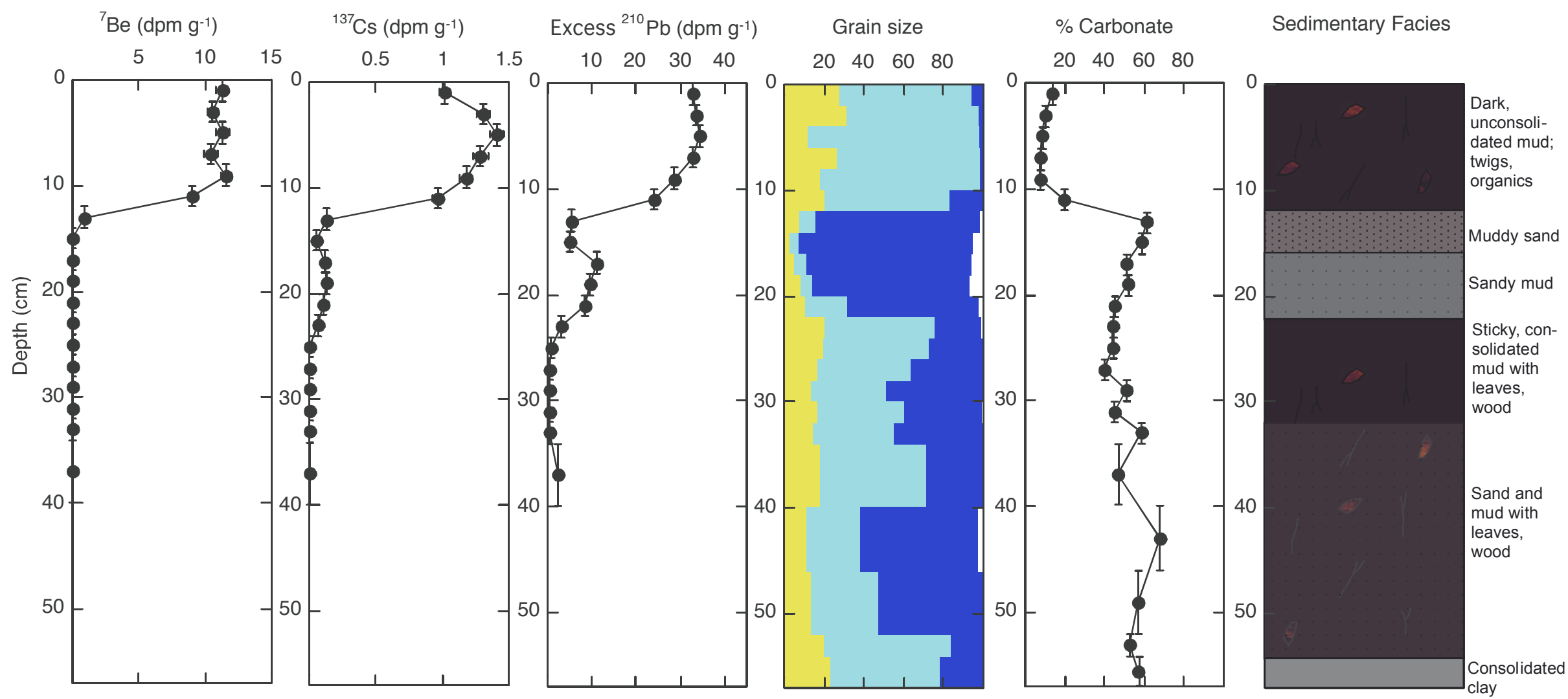

Figure 15. Sedimentary facies and isotope activity at Station F in June 2006 (Core K0606-F). Activities of 7 Be, ${ }^{137}$ Cs, and excess $210 P b$, all decay-corrected to the date of sample collection, are shown in disintegrations per minute (dpm) per dry gram of salt-free sediment. This site is in the Black Hole. 


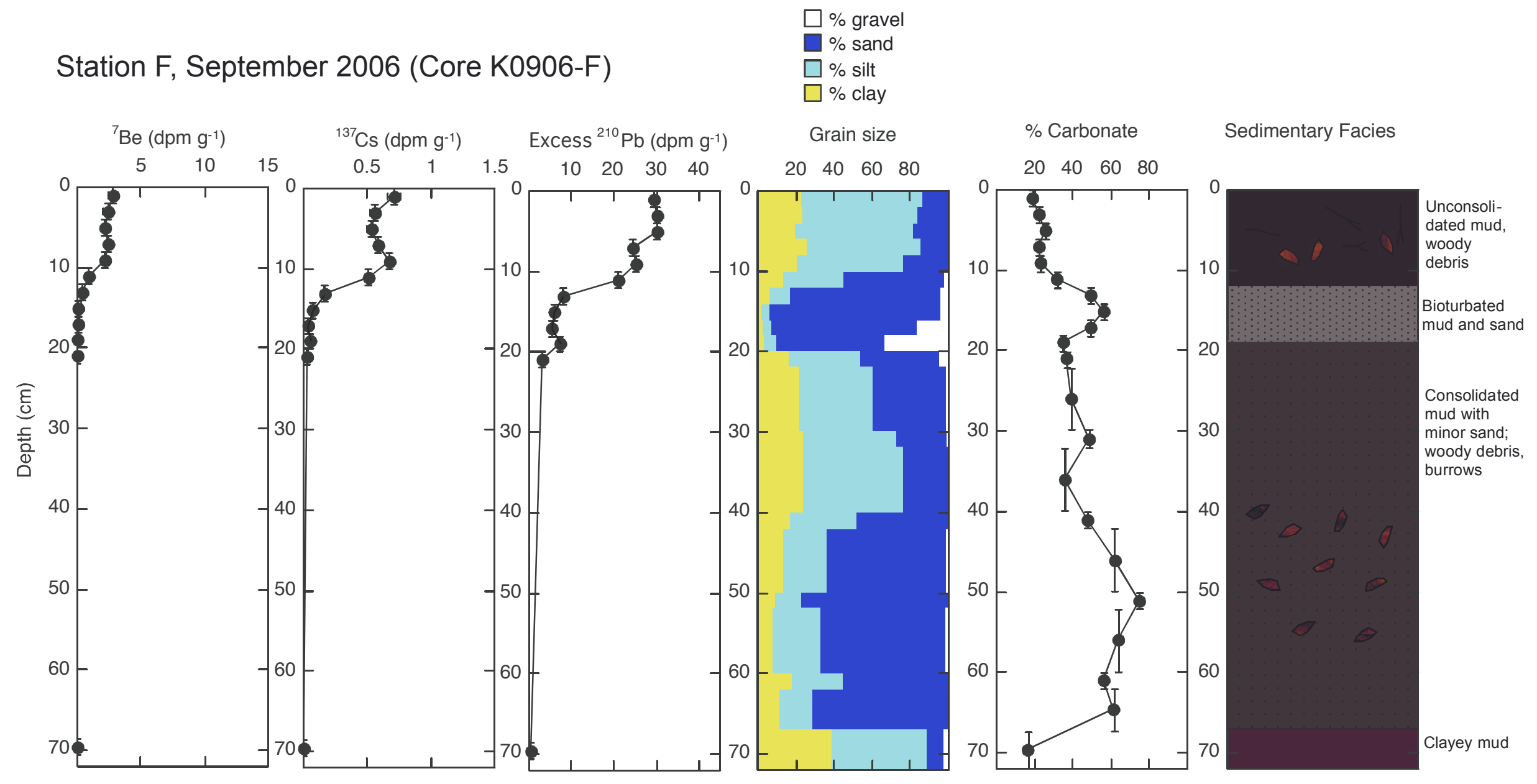

Figure 16. Sedimentary facies and isotope activity at Station F in September 2006 (Core K0906-F). Activities of 7Be, 137Cs, and excess 210Pb, all decay-corrected to the date of sample collection, are shown in disintegrations per minute $(\mathrm{dpm})$ per dry gram of salt-free sediment. This site is in the Black Hole. 


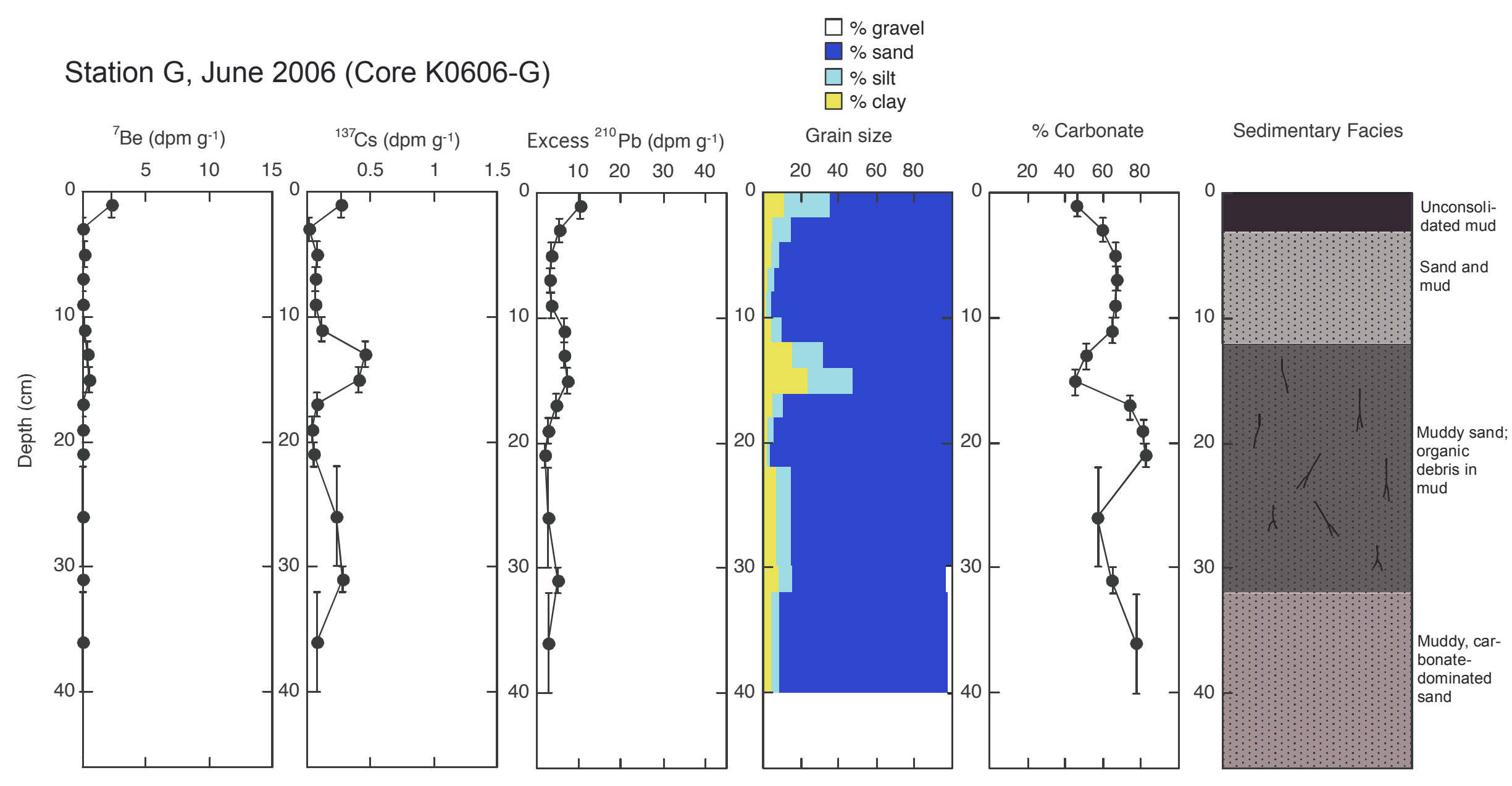

Figure 17. Sedimentary facies and isotope activity at Station G in September 2006 (Core K0906-G). Activities of 7Be, 137Cs, and excess 210Pb, all decaycorrected to the date of sample collection, are shown in disintegrations per minute (dpm) per dry gram of salt-free sediment. This site is next to the reef wall on the eastern side of Hanalei Bay, near the river mouth. 


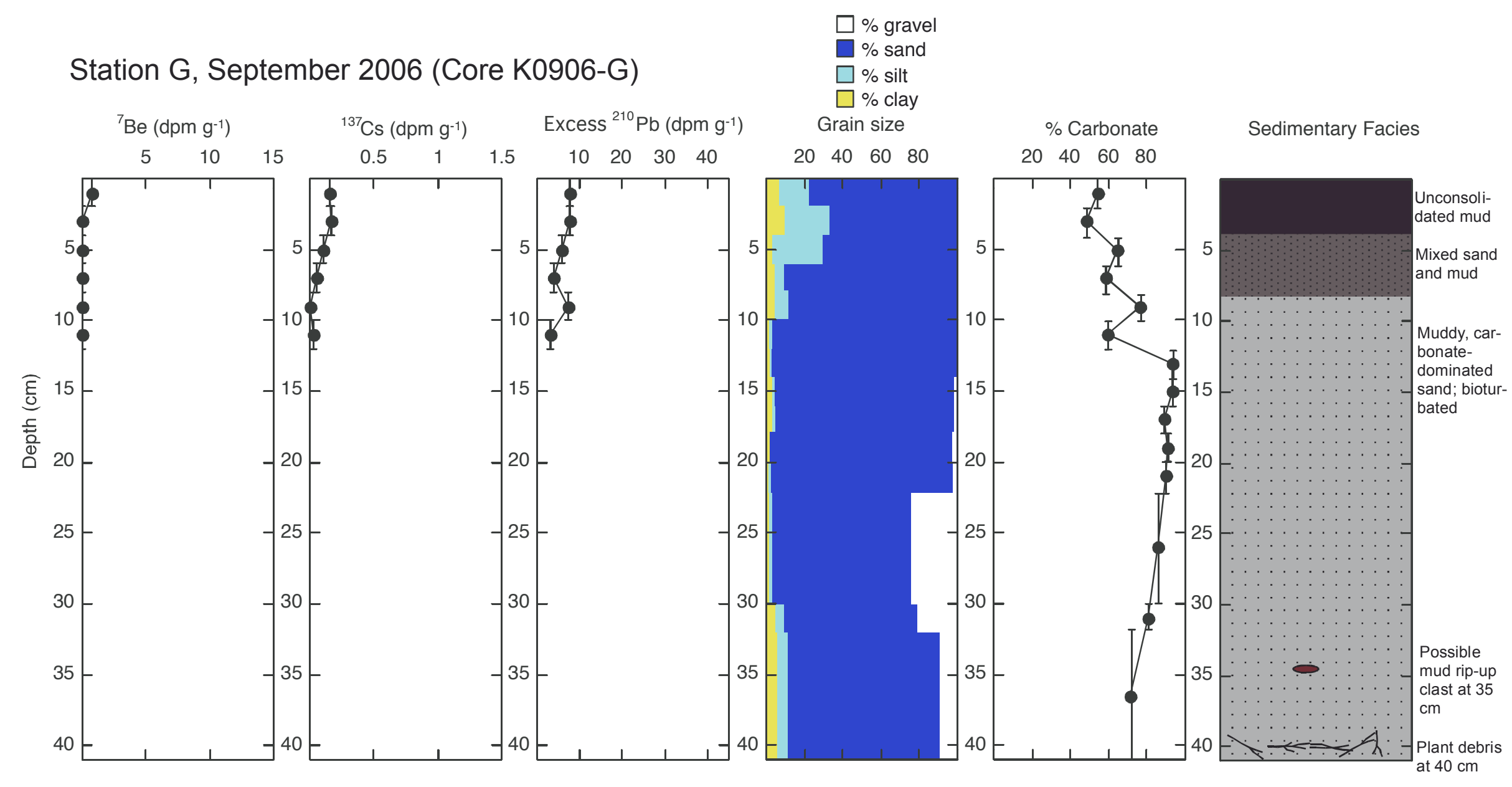

Figure 18. Sedimentary facies and isotope activity at Station G in September 2006 (Core K0906-G). Activities of 7Be, 137Cs, and excess 210Pb, all decaycorrected to the date of sample collection, are shown in disintegrations per minute (dpm) per dry gram of salt-free sediment. This site is next to the reef wall on the eastern side of Hanalei Bay, near the river mouth. 


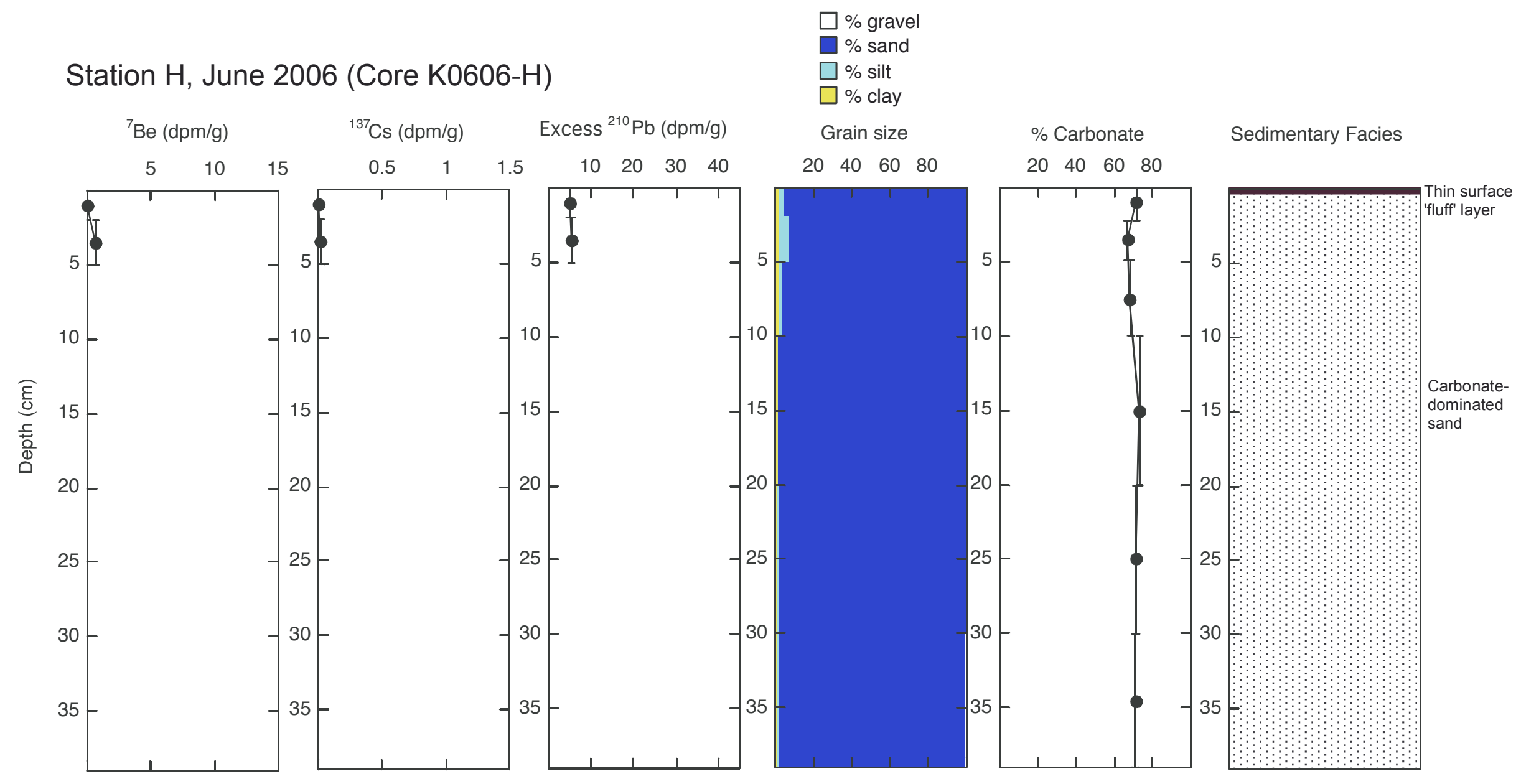

Figure 19. Sedimentary facies and isotope activity at Station H in June 2006 (Core K0606-H). Activities of $7 \mathrm{Be}$, ${ }^{137 C s}$, and excess 210Pb, all decay-corrected to the date of sample collection, are shown in disintegrations per minute $(d p m)$ per dry gram of salt-free sediment. This site is south of the Black Hole, on the southeastern side of Hanalei Bay. 


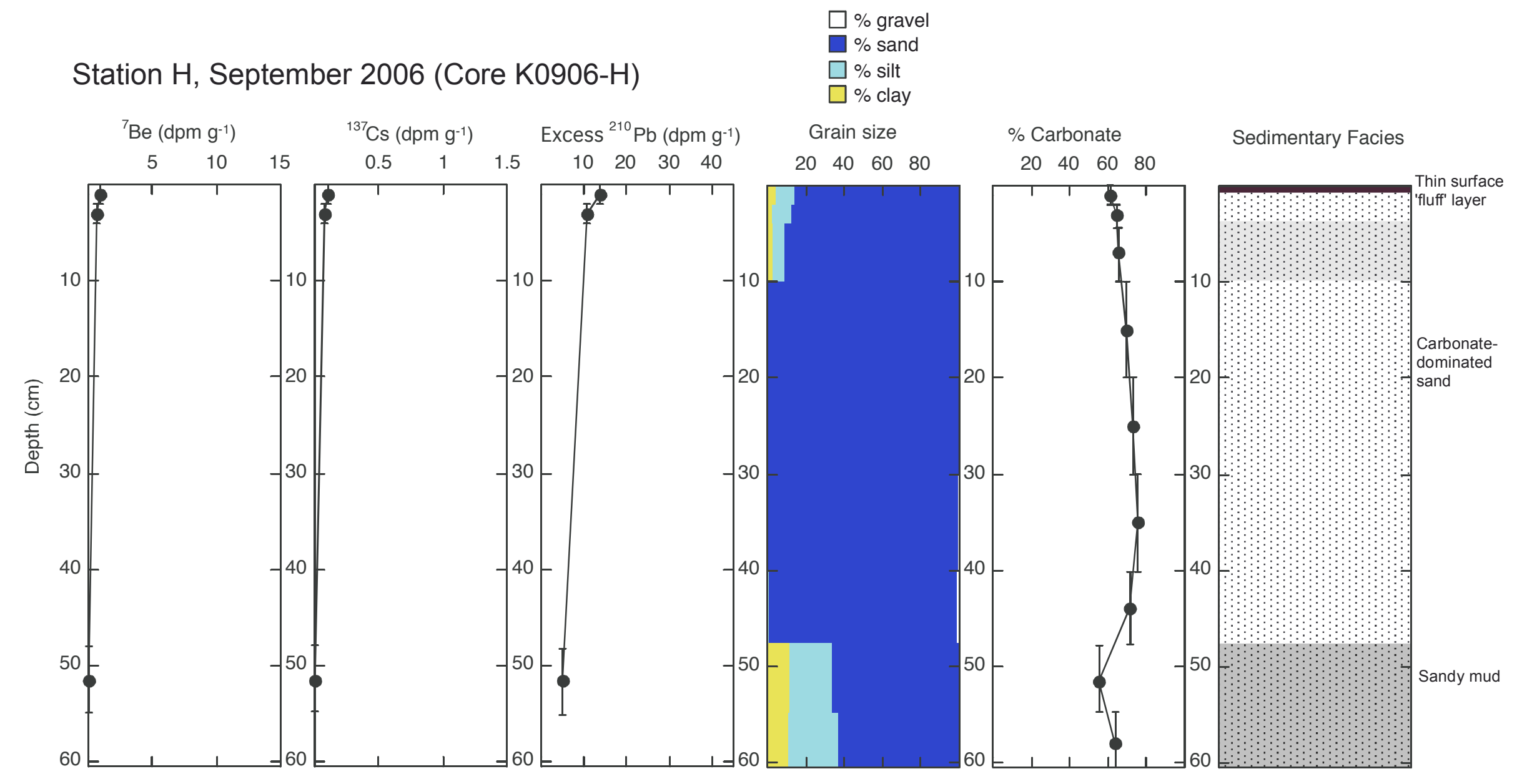

Figure 20. Sedimentary facies and isotope activity at Station H in September 2006 (Core K0906-H). Activities of 7Be, 137Cs, and excess 210Pb, all decaycorrected to the date of sample collection, are shown in disintegrations per minute (dpm) per dry gram of salt-free sediment. This site is south of the Black Hole, on the southeastern side of Hanalei Bay. 
Station I, June 2006 (Core K0606-I)

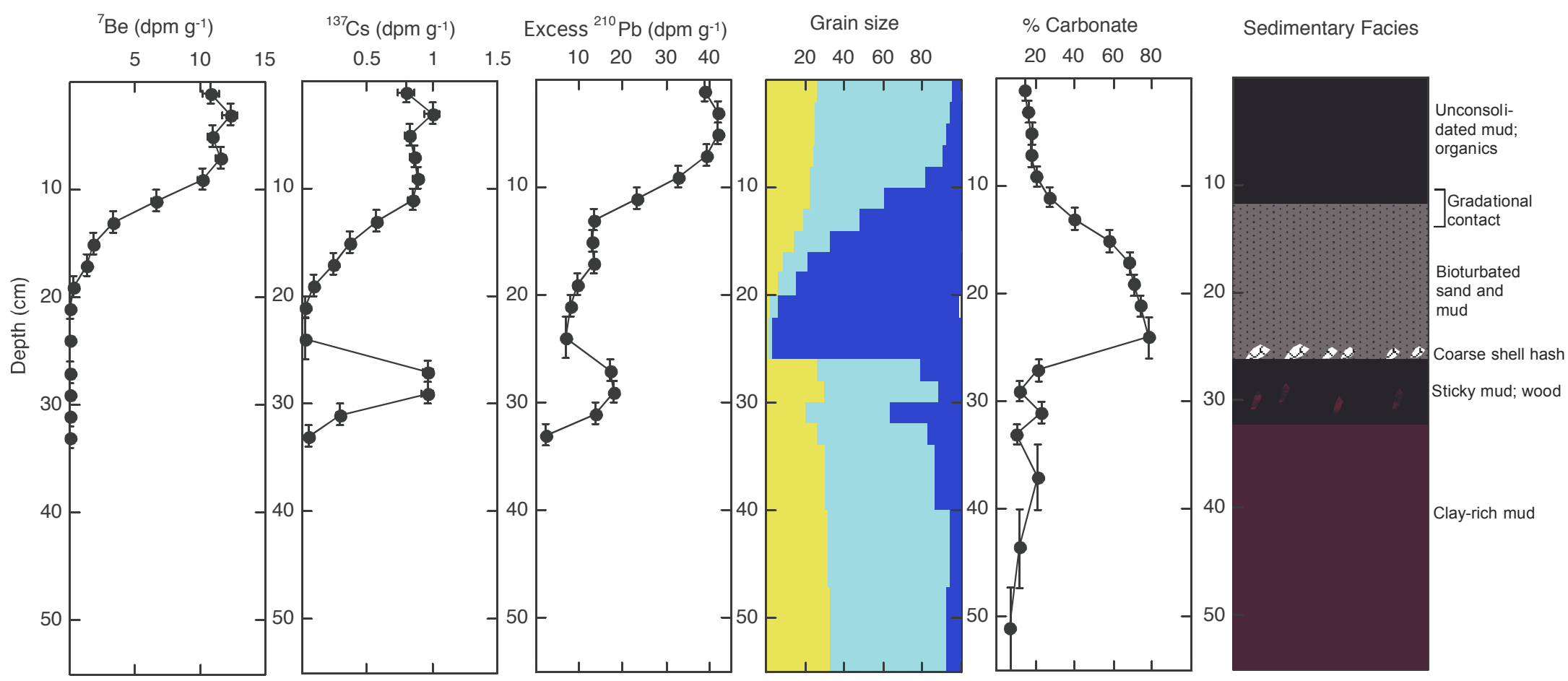

Figure 21. Sedimentary facies and isotope activity at Station I in June 2006 (Core K0606-I). Activities of 7Be, 137 Cs, and excess $210 \mathrm{~Pb}$, all decay-corrected to the date of sample collection, are shown in disintegrations per minute $(\mathrm{dpm})$ per dry gram of salt-free sediment. This site is in the Black Hole. 

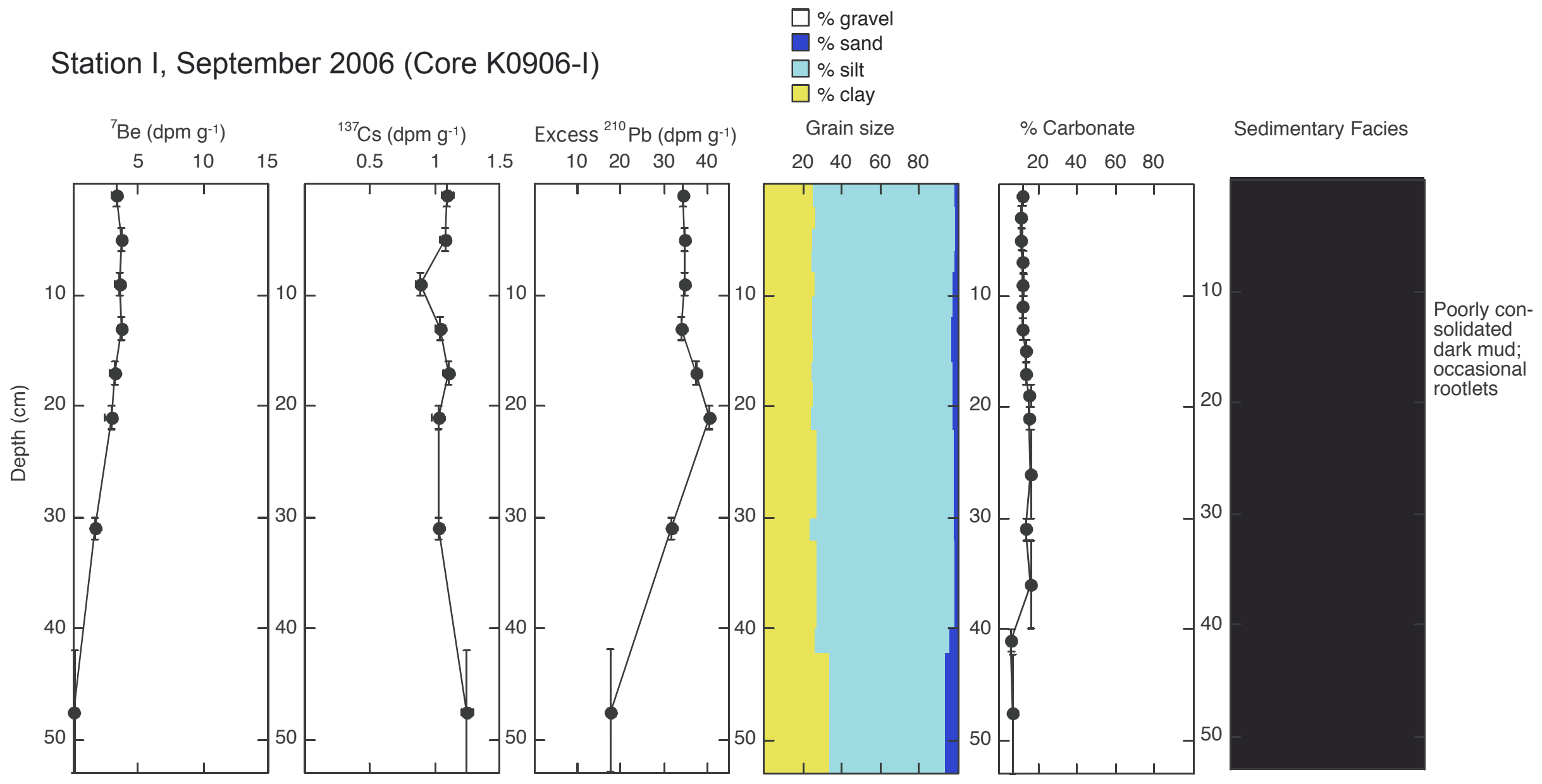

Figure 22. Sedimentary facies and isotope activity at Station I in September 2006 (Core K0906-I). Activities of 7Be, 137Cs, and excess 210Pb, all decay-corrected to the date of sample collection, are shown in disintegrations per minute (dpm) per dry gram of salt-free sediment. This site is in the Black Hole. 


\section{Station A, June 2006 (Core K0606-A)}

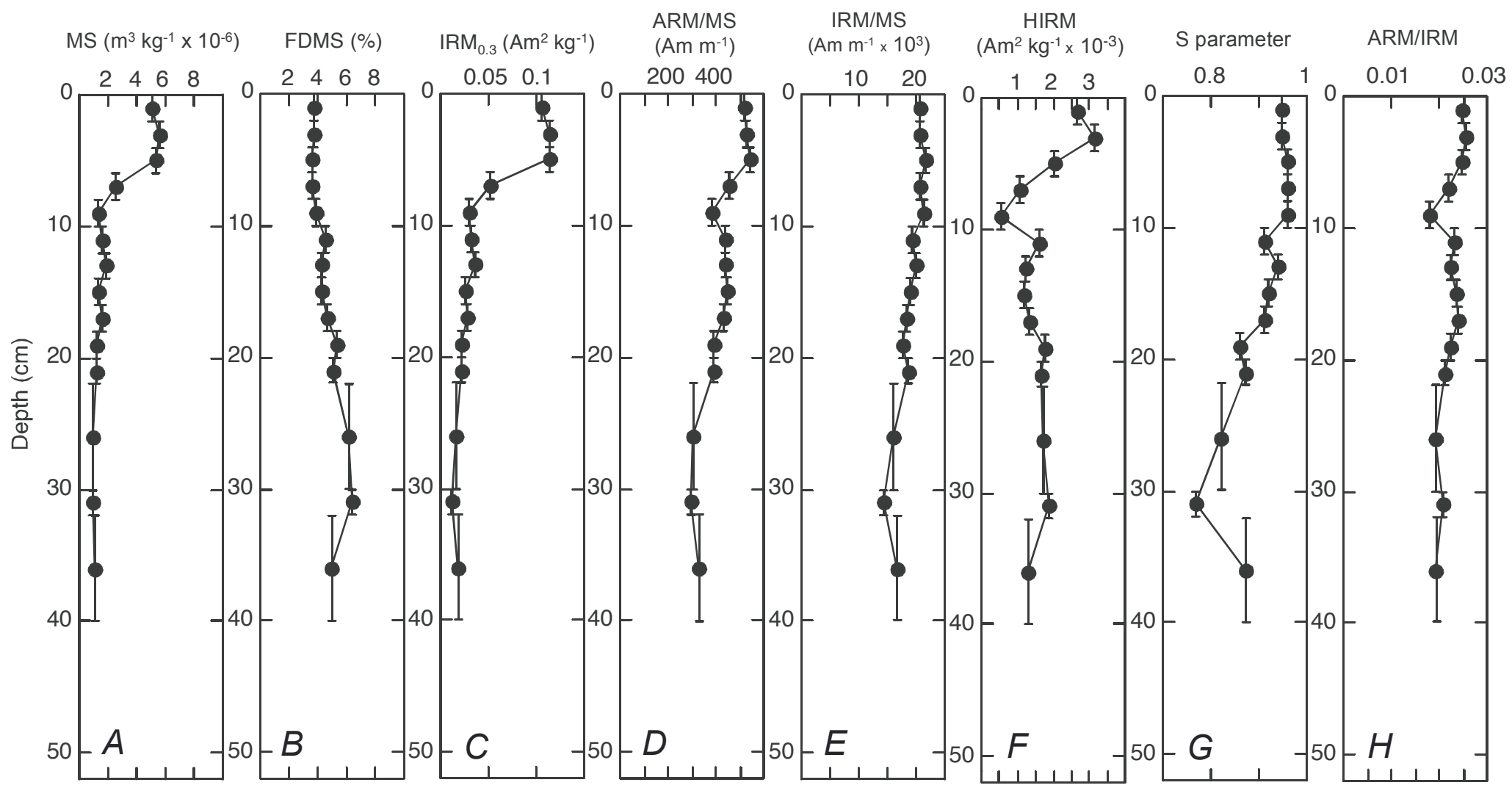

Figure 23. Magnetic properties measured at Site $A$ in June 2006. (A) low-frequency magnetic susceptibility (MS), a measure of all magnetic minerals; (B) frequency-dependent magnetic susceptibility (FDMS), a measure of superparamagnetic $(<30 \mathrm{~nm}) \mathrm{magne-}$ tite and (or) maghematite; (C) isothermal remanent magnetization (IRM) induced at $0.3 \mathrm{~T}$, a measure of magnetite content with magnetic grain size >30 $\mathrm{nm} ;(D)$ ratio of anhysteretic remanent magnetization (ARM, a measure of magnetite with fine magnetic grain size) to MS; (E) ratio of IRM to MS; (F) hard isothermal remanent magnetization (HIRM, with higher values indicating higher hematite content); (G) S parameter (ratio of IRM acquired at $0.3 T$ with IRM acquired at $1.2 T$ ), with higher values indicating increasing magnetite relative to hematite; and $(H)$ ratio of ARM to IRM, with higher values indicating finer magnetic grain size. 


\section{Station A, September 2006 (Core K0906-A)}

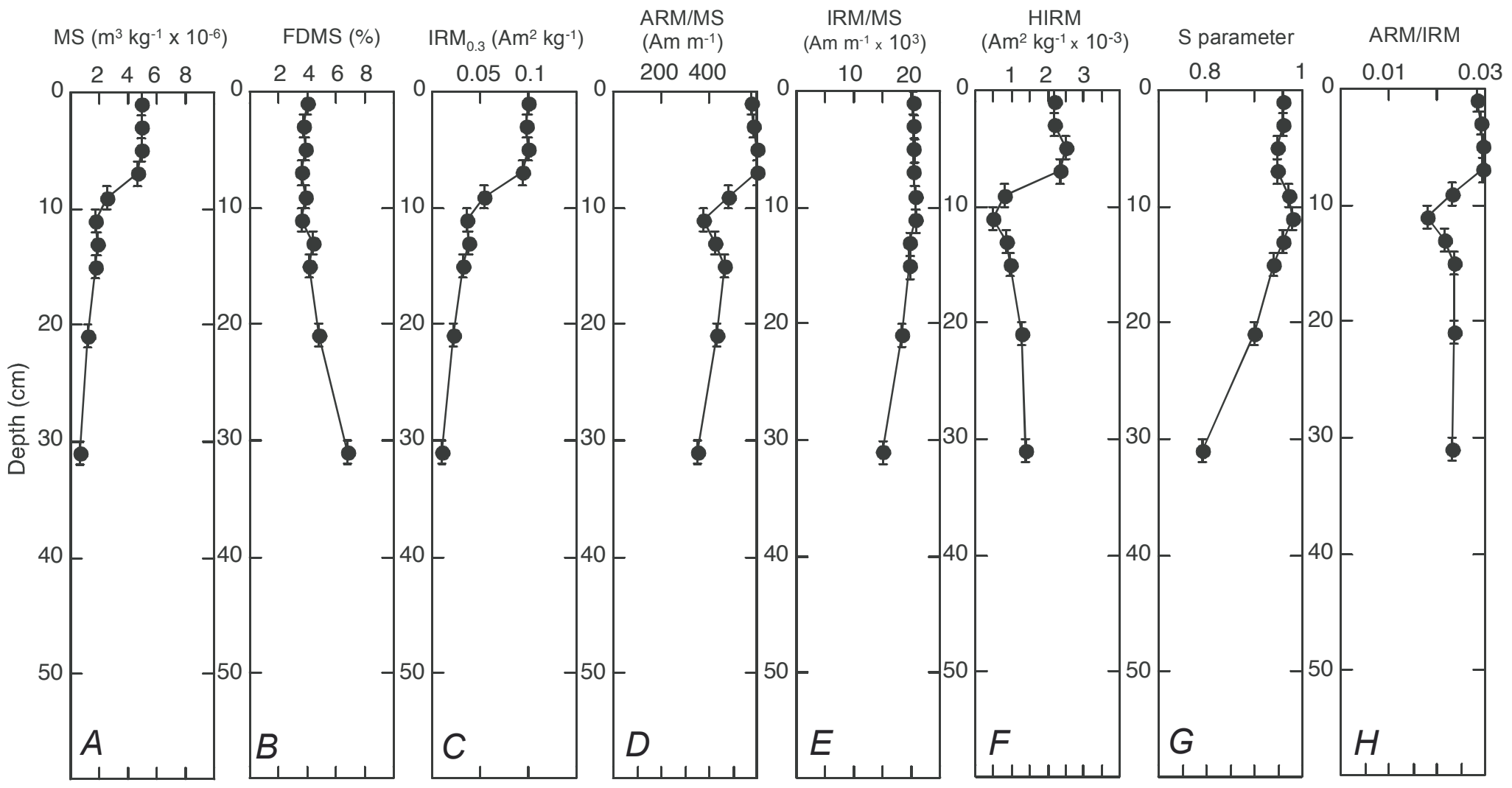

Figure 24. Magnetic properties measured at Site A in September 2006. (A) low-frequency magnetic susceptibility (MS), a measure of all magnetic minerals; (B) frequency-dependent magnetic susceptibility (FDMS), a measure of superparamagnetic (<30 nm) magnetite and (or) maghematite; (C) isothermal remanent magnetization (IRM) induced at 0.3 T, a measure of magnetite content with magnetic grain size >30 nm; (D) ratio of anhysteretic remanent magnetization (ARM, a measure of magnetite with fine magnetic grain size) to MS; (E) ratio of IRM to MS; (F) hard isothermal remanent magnetization (HIRM, with higher values indicating higher hematite content); (G) S parameter (ratio of IRM acquired at $0.3 T$ with IRM acquired at $1.2 T$ ), with higher values indicating increasing magnetite relative to hematite; and $(H)$ ratio of ARM to IRM, with higher values indicating finer magnetic grain size. 


\section{Station C, June 2006 (Core K0606-C)}

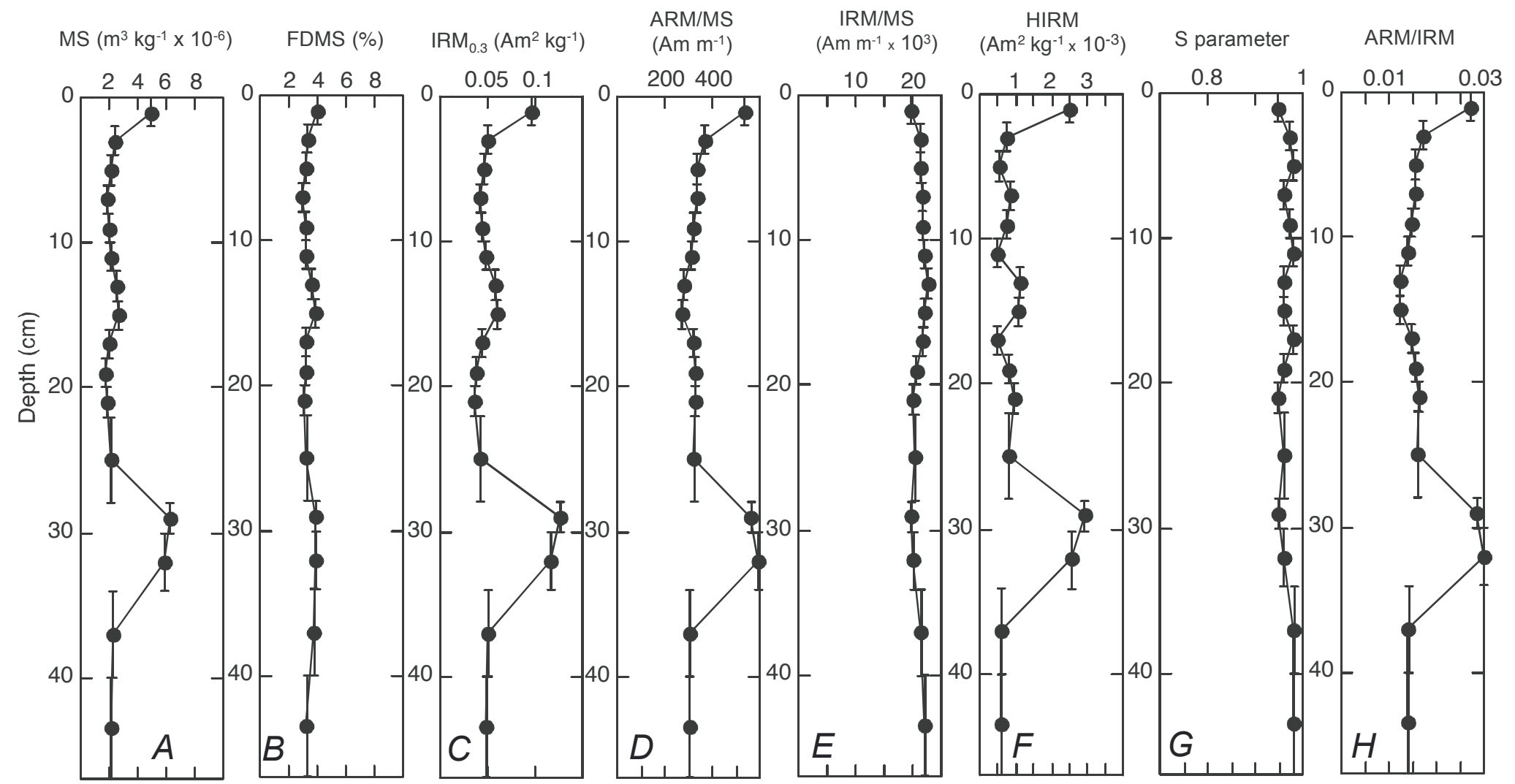

Figure 25. Magnetic properties measured at Site C in June 2006. (A) low-frequency magnetic susceptibility (MS), a measure of all magnetic minerals; (B) frequency-dependent magnetic susceptibility (FDMS), a measure of superparamagnetic (<30 nm) magnetite and (or) maghematite; (C) isothermal remanent magnetization (IRM) induced at 0.3 T, a measure of magnetite content with magnetic grain size $>30 \mathrm{~nm}$; (D) ratio of anhysteretic remanent magnetization (ARM, a measure of magnetite with fine magnetic grain size) to MS; (E) ratio of IRM to MS; (F) hard isothermal remanent magnetization (HIRM, with higher values indicating higher hematite content); (G) S parameter (ratio of IRM acquired at $0.3 T$ with IRM acquired at $1.2 T$ ), with higher values indicating increasing magnetite relative to hematite; and $(H)$ ratio of $A R M$ to IRM, with higher values indicating finer magnetic grain size. 


\section{Station C, September 2006 (Core K0906-C)}

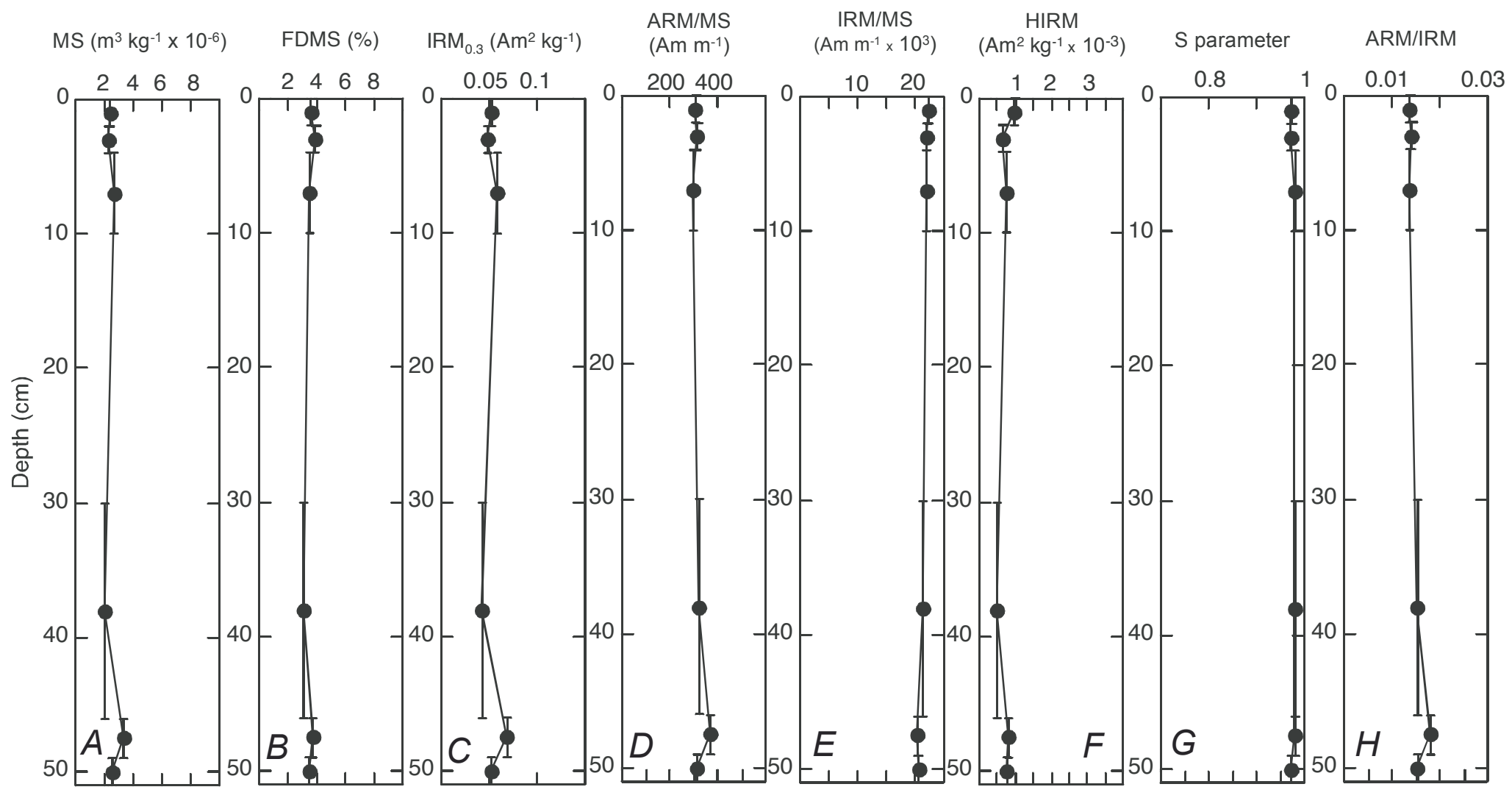

Figure 26. Magnetic properties measured at Site C in September 2006. (A) low-frequency magnetic susceptibility (MS), a measure of all magnetic minerals; (B) frequency-dependent magnetic susceptibility (FDMS), a measure of superparamagnetic (<30 nm) magnetite and (or) maghematite; (C) isothermal remanent magnetization (IRM) induced at 0.3 T, a measure of magnetite content with magnetic grain size $>30 \mathrm{~nm}$; (D) ratio of anhysteretic remanent magnetization (ARM, a measure of magnetite with fine magnetic grain size) to MS; (E) ratio of IRM to MS; (F) hard isothermal remanent magnetization (HIRM, with higher values indicating higher hematite content); (G) S parameter (ratio of IRM acquired at $0.3 T$ with IRM acquired at 1.2 T), with higher values indicating increasing magnetite relative to hematite; and $(H)$ ratio of $A R M$ to IRM, with higher values indicating finer magnetic grain size. 


\section{Station I, June 2006 (Core K0606-I)}

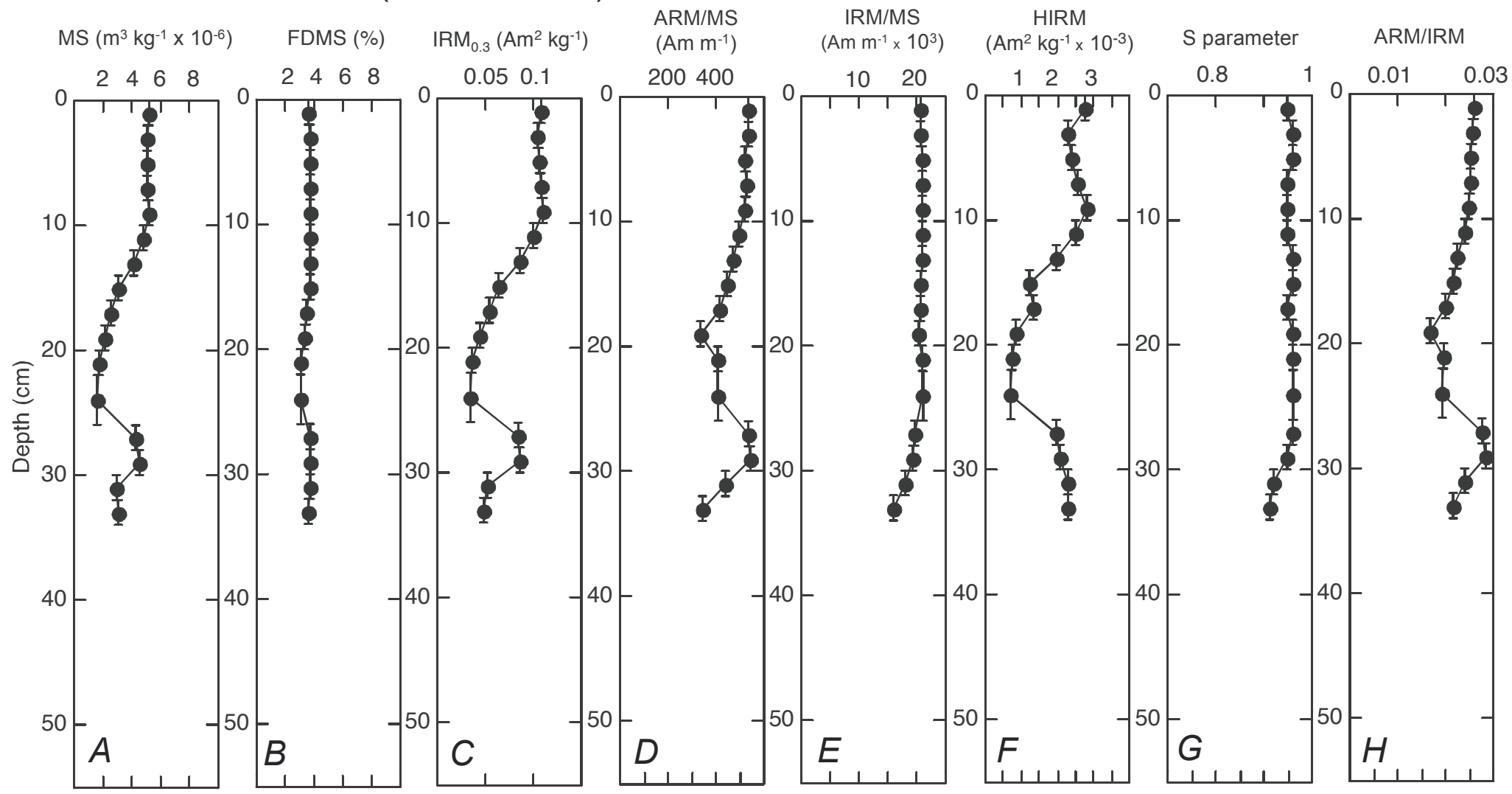

Figure 27. Magnetic properties measured at Site I in June 2006. (A) low-frequency magnetic susceptibility (MS), a measure of all magnetic minerals; (B) frequency-dependent magnetic susceptibility (FDMS), a measure of superparamagnetic $(<30 \mathrm{~nm})$ magnetite and (or) maghematite; (C) isothermal remanent magnetization (IRM) induced at $0.3 \mathrm{~T}$, a measure of magnetite content with magnetic grain size >30 nm; (D) ratio of anhysteretic remanent magnetization (ARM, a measure of magnetite with fine magnetic grain size) to MS; (E) ratio of IRM to MS; (F) hard isothermal remanent magnetization (HIRM, with higher values indicating higher hematite content); (G) S parameter (ratio of IRM acquired at $0.3 T$ with IRM acquired at $1.2 T$ ), with higher values indicating increasing magnetite relative to hematite; and $(H)$ ratio of ARM to IRM, with higher values indicating finer magnetic grain size. 


\section{Station I, September 2006 (Core K0906-I)}

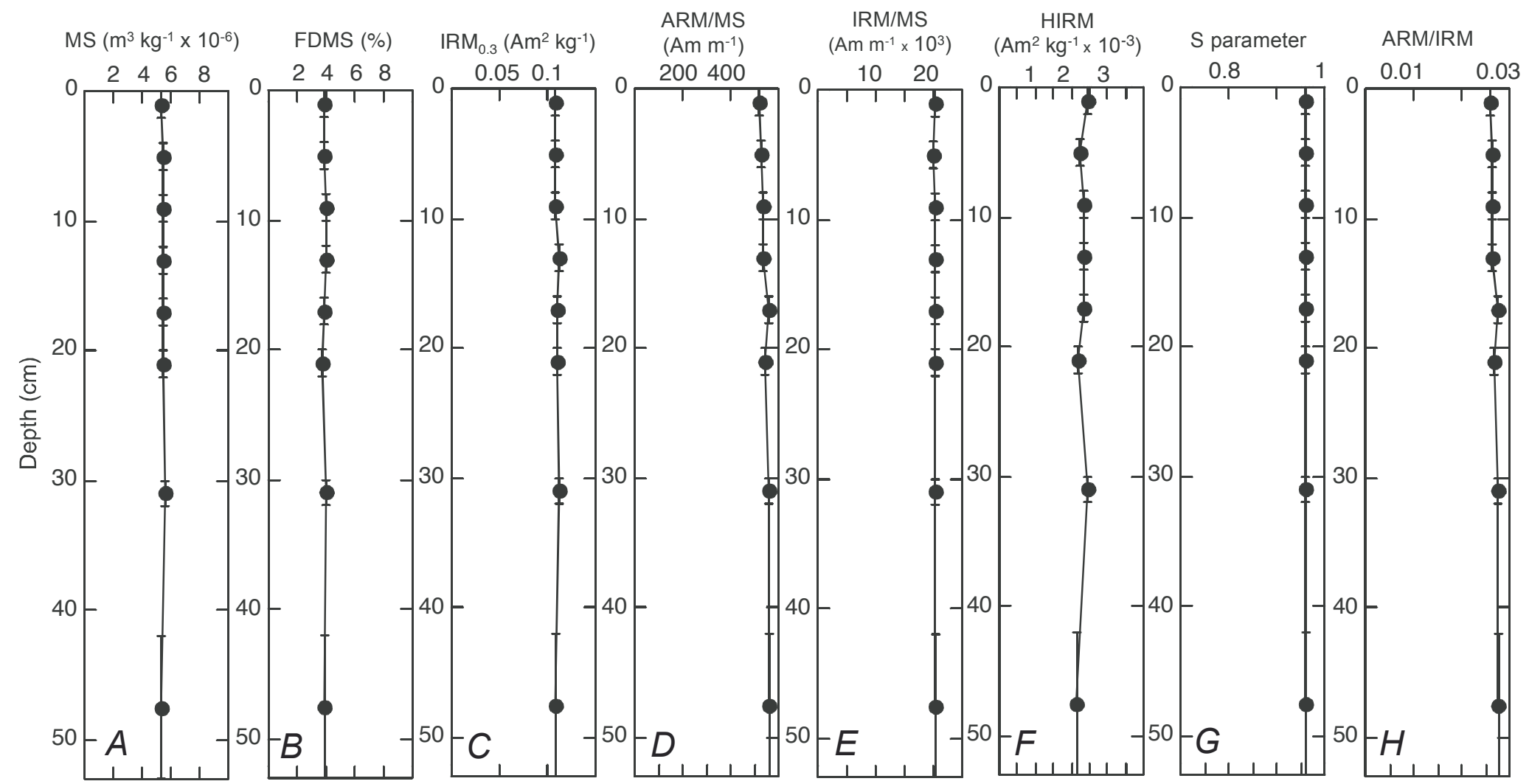

Figure 28. Magnetic properties measured at Site I in September 2006. (A) low-frequency magnetic susceptibility (MS), a measure of all magnetic minerals; (B) frequency-dependent magnetic susceptibility (FDMS), a measure of superparamagnetic $(<30 \mathrm{~nm})$ magnetite and (or) maghematite; (C) isothermal remanent magnetization (IRM) induced at $0.3 \mathrm{~T}$, a measure of magnetite content with magnetic grain size $>30 \mathrm{~nm}$; (D) ratio of anhysteretic remanent magnetization (ARM, a measure of magnetite with fine magnetic grain size) to MS; (E) ratio of IRM to MS; $(F)$ hard isothermal remanent magnetization (HIRM, with higher values indicating higher hematite content); (G) S parameter (ratio of IRM acquired at $0.3 T$ with IRM acquired at 1.2 T), with higher values indicating increasing magnetite relative to hematite; and $(H)$ ratio of ARM to IRM, with higher values indicating finer magnetic grain size. 
CRAMP site, on the western side of the bay, had ${ }^{7} \mathrm{Be}$ activity of $5-6 \mathrm{dpm} \mathrm{g}^{-1},{ }^{137} \mathrm{Cs}$ from $0.15-0.19$ dpm g ${ }^{-1}$, and excess ${ }^{210} \mathrm{~Pb}$ ranging from $67-78 \mathrm{dpm} \mathrm{g}^{-1}$. At the Northeast Dobie site, northeast of Hanalei Bay (fig. $2 A$ ), ${ }^{7} \mathrm{Be}$ and ${ }^{137} \mathrm{Cs}$ were virtually absent from sediment collected in a tube trap, whereas excess ${ }^{210} \mathrm{~Pb}$ was detected at $\sim 4-24 \mathrm{dpm} \mathrm{g}^{-1}$.

Isotope activities in three samples collected from the Hanalei River bed provide a basis for comparison with samples collected in the bay. The three river-bed samples spanned approximately the lowermost $4 \mathrm{~km}$ of the river channel. The sample collected farthest upstream (River Site 1 [RS1]; table 3) is $\sim 4 \mathrm{~km}$ from the mouth of the river and above most of the agricultural runoff in the river valley. Sediment from RS1 in June 2006 consisted of coarse sand with minor mud. This sample contained ${ }^{7} \mathrm{Be}$ activity of $0.36 \pm 0.07 \mathrm{dpm} \mathrm{g}^{-1},{ }^{137} \mathrm{Cs}$ activity of $0.56 \pm 0.02 \mathrm{dpm} \mathrm{g}^{-1}$, and excess ${ }^{210} \mathrm{~Pb}$ activity of $0.1 \pm 0.05 \mathrm{dpm} \mathrm{g}^{-1}$. In September 2006, RS1 contained no measurable ${ }^{7} \mathrm{Be}$ activity, ${ }^{137} \mathrm{Cs}$ activity of $1.57 \pm 0.03 \mathrm{dpm} \mathrm{g}^{-1}$, and excess ${ }^{210} \mathrm{~Pb}$ activity of $1.46 \pm 0.08 \mathrm{dpm} \mathrm{g}^{-1}$. For comparison, in August 2005, before the large floods of early 2006, the river bed at RS1 was composed of mud and organic debris with ${ }^{7} \mathrm{Be}$ activity of $0.22 \pm 0.06 \mathrm{dpm} \mathrm{g}^{-1},{ }^{137} \mathrm{Cs}$ activity of 0.39 $\pm 0.02 \mathrm{dpm} \mathrm{g}^{-1}$, and excess ${ }^{210} \mathrm{~Pb}$ activity of $0.09 \pm 0.05 \mathrm{dpm} \mathrm{g}^{-1}$ (Draut and others, 2006).

Sediment at River Site 2 (RS2), located $\sim 2.5 \mathrm{~km}$ above the river mouth, contained consolidated mud and organic matter in the river bank. In June, 2006, this sediment contained ${ }^{7} \mathrm{Be}$ activity of $0.87 \pm 0.1 \mathrm{dpm} \mathrm{g}^{-1},{ }^{137} \mathrm{Cs}$ activity of $0.79 \pm 0.02 \mathrm{dpm} \mathrm{g}^{-1}$, and excess ${ }^{210} \mathrm{~Pb}$ activity of 1.45 $\pm 0.07 \mathrm{dpm} \mathrm{g}^{-1}$. In September 2006, sediment from RS2 contained no measurable ${ }^{7} \mathrm{Be}$ activity, ${ }^{137} \mathrm{Cs}$ activity of $1.80 \pm 0.04 \mathrm{dpm} \mathrm{g}^{-1}$, and excess ${ }^{210} \mathrm{~Pb}$ activity of $4.09 \pm 0.12 \mathrm{dpm} \mathrm{g}^{-1}$. For comparison, in August 2005, sediment at RS2 consisted of mud and organic material with ${ }^{7} \mathrm{Be}$ activity of $0.06 \pm$ $0.03 \mathrm{dpm} \mathrm{g}^{-1},{ }^{137} \mathrm{Cs}$ activity of $0.74 \pm 0.02 \mathrm{dpm} \mathrm{g}^{-1}$, and excess ${ }^{210} \mathrm{~Pb}$ activity of $1.44 \pm 0.09 \mathrm{dpm} \mathrm{g}^{-1}$ (Draut and others, 2006).

River Site 3 (RS3), located $\sim 0.8 \mathrm{~km}$ upstream from the river mouth, was sampled in 2005 (Draut and others, 2006) but was not re-occupied for sampling in 2006 because sediment at that site was too coarse-grained to facilitate comparison of its isotope signature with other sites.

River Site 4 (RS4) is at the river mouth, where fine sand and mud were collected on the left (southern) bank of the river by the Hanalei beach park. In June 2006, sediment at RS4 contained ${ }^{7}$ Be activity of $0.145 \pm 0.034 \mathrm{dpm} \mathrm{g}^{-1},{ }^{137} \mathrm{Cs}$ activity of $0.015 \pm 0.004 \mathrm{dpm} \mathrm{g}^{-1}$, and excess ${ }^{210} \mathrm{~Pb}$ activity of $1.42 \pm 0.05 \mathrm{dpm} \mathrm{g}^{-1}$. In September 2006, RS4 contained ${ }^{7} \mathrm{Be}$ activity of $0.249 \pm 0.060$ dpm g ${ }^{-1},{ }^{137} \mathrm{Cs}$ activity of $0.004 \pm 0.003 \mathrm{dpm} \mathrm{g} \mathrm{g}^{-1}$, and excess ${ }^{210} \mathrm{~Pb}$ activity of $1.70 \pm 0.06 \mathrm{dpm} \mathrm{g}^{-1}$. For comparison, in August 2005, fine sand from RS4 contained ${ }^{7}$ Be activity of $0.055 \pm 0.027 \mathrm{dpm}$ $\mathrm{g}^{-1},{ }^{137} \mathrm{Cs}$ activity of $0.003 \pm 0.002 \mathrm{dpm} \mathrm{g}^{-1}$, and excess ${ }^{210} \mathrm{~Pb}$ activity of $2.11 \pm 0.076 \mathrm{dpm} \mathrm{g}^{-1}$. Because the river-sediment samples were grab samples from the bed surface only, isotope inventories in the river bed beneath those sampling sites cannot be measured.

The sample of suspended sediment collected from Hanalei River water on September 6, 2006, contained (in the suspended solids) ${ }^{7} \mathrm{Be}$ activity of $23.8 \pm 1.57 \mathrm{dpm} \mathrm{g}{ }^{-1},{ }^{137} \mathrm{Cs}$ activity of 1.66 $\pm 0.16 \mathrm{dpm} \mathrm{g}^{-1}$, and excess ${ }^{210} \mathrm{~Pb}$ activity of $9.96 \pm 0.70 \mathrm{dpm} \mathrm{g}^{-1}$. Colloidal material in the same sample contained ${ }^{7} \mathrm{Be}$ activity of $36.2 \pm 7.81 \mathrm{dpm} \mathrm{g}^{-1},{ }^{137} \mathrm{Cs}$ activity of $3.15 \pm 0.53 \mathrm{dpm} \mathrm{g}^{-1}$, and excess ${ }^{210} \mathrm{~Pb}$ activity of $18.9 \pm 2.22 \mathrm{dpm} \mathrm{g}^{-1}$.

Comparisons of magnetic properties with sedimentary properties reveal some fundamental associations in these near-shore marine settings in which carbonate sediment is mixed with volcanogenic sediment. First, the amount of magnetite shows an expected inverse correspondence with the amount of nonmagnetic carbonate. Because much of the carbonate in these cores occurs in the sand fraction, magnetite abundance is also related roughly to bulk sediment grain size, with increasing magnetite associated with increasing amounts of fine-grained sediment (silt and clay). 
Second, finer magnetic grain sizes, as expressed by increasing values of ARM/IRM, correspond with finer bulk sediment grain size (higher silt and clay content), indicating that the sizes of magnetic particles overall are controlled by the sizes of terrigenous sediment particles. (Although this observation may seem obvious, it is not always the case for volcanogenic sediment because magnetic particles are commonly contained in larger rock fragments.) Both associations are illustrated by comparing respective data for Sites A, C, and I for June 2006 (fig. 5 with fig. 23, 9 with 25, and 21 with 27).

Magnetic properties vary widely, taking results from all analyzed 2005 and 2006 cores (appendix 3). Both magnetite and hematite abundances vary by about an order of magnitude (0.011-0.125 $\mathrm{Am}^{2} \mathrm{~kg}^{-1}$ in IRM and 0.23-3.13 $\mathrm{x} 10^{-3} \mathrm{Am}^{2} \mathrm{~kg}^{-1}$ in HIRM, respectively). Concentration-independent properties also vary widely: ARM/MS between 237 and $598 \mathrm{Am}^{-1}$; ARM/IRM between 0.012 and 0.030; FDMS between 3.0 and 7.0 percent; and S parameter between 0.74 (very high in hematite) and 0.98 (very high in magnetite). Samples from recent flood deposits, defined here as having IRM $>0.08 \mathrm{Am}^{2} \mathrm{~kg}^{-1}$, and corroborated by other properties and visual observations of cored sediment, show restricted ranges in all magnetic properties: IRM, 0.085-0.125 $\mathrm{Am}^{2} \mathrm{~kg}^{-1}$; HIRM, 1.96-3.13 x 10-3 $\mathrm{Am}^{2} \mathrm{~kg}^{-1}$; ARM/MS, 470-598 Am Am $^{-1}$ ARM/IRM, 0.022-0.030; FDMS, 3.6-4.0 percent and S parameter, 0.95-0.96.

Magnetic and isotopic properties are closely similar among recent flood deposits in cores, sediment collected by sediment traps in the vicinity of the Black Hole, and the sample of suspended river sediment collected on September 6, 2006. The ${ }^{137}$ Cs activity in all recent (2006) flood sediments (average of $\left.1.05 \mathrm{dpm} \mathrm{g}^{-1}\right)$ is close to that in fluvial suspended sediment $\left(1.66 \mathrm{dpm} \mathrm{g}^{-1}\right)$ and higher than the range for sediment collected in the marine sediment traps $\left(0.66-1.05 \mathrm{dpm} \mathrm{g}^{-1}\right.$; average, $0.91 \mathrm{dpm} \mathrm{g}^{-1}$ ). Sediments in other traps (CRAMP, SC, and NE sites; locations shown in fig. 2A) deployed in the bay contain lower amounts of magnetite and hematite (average IRM of $0.055 \mathrm{Am}^{2} \mathrm{~kg}^{-1}$; average HIRM of $0.99 \times 10^{-3} \mathrm{Am}^{2} \mathrm{~kg}^{-1}$ ) perhaps caused by sorting of these highdensity minerals with distance from the river mouth. Sediments at these sites remote from the river mouth are also relatively low in ${ }^{137} \mathrm{Cs}\left(0-0.29 \mathrm{dpm} \mathrm{g}^{-1}\right.$; average activity of $\left.0.15 \mathrm{dpm} \mathrm{g}^{-1}\right)$.

\section{Discussion}

\section{Sedimentary Facies, Isotopic, and Magnetic Signatures}

Terrestrial sediment can be fairly easily distinguished from marine-sourced sediment in Hanalei Bay sediment cores. Terrestrial (flood) sediment is typically dark brown or black in color, dominated by silt and clay grain sizes, and contains a lower proportion of carbonate (and correspondingly higher magnetic susceptibility, where measured) than the coarser-grained, lightcolored carbonate-rich marine deposits (fig. 29). Sediment with an inferred terrigenous origin (based on those criteria) that also contains appreciable ${ }^{7} \mathrm{Be}$ is interpreted as having been deposited less than eight months before the time of core collection. Sediment rich in ${ }^{137} \mathrm{Cs}$ is similarly interpreted as having a terrigenous origin; sediment containing ${ }^{137} \mathrm{Cs}$ but lacking ${ }^{7} \mathrm{Be}$ is interpreted as terrigenous sediment deposited more than eight months ago, such that any ${ }^{7} \mathrm{Be}$ it may have contained was lost through radioactive decay before core collection. High levels of ${ }^{7} \mathrm{Be}$ and ${ }^{137} \mathrm{Cs}$ in the fluvial suspended sediment and colloidal material support those interpretations. Sediment samples collected from the river bed did not contain as much ${ }^{7} \mathrm{Be}$ and ${ }^{137} \mathrm{Cs}$ as the fluvial suspended sediment. This is assumed to be either because older, relict fluvial bed sediment was sampled or because the grain size of the bed sediment was much coarser than the suspended sediment 

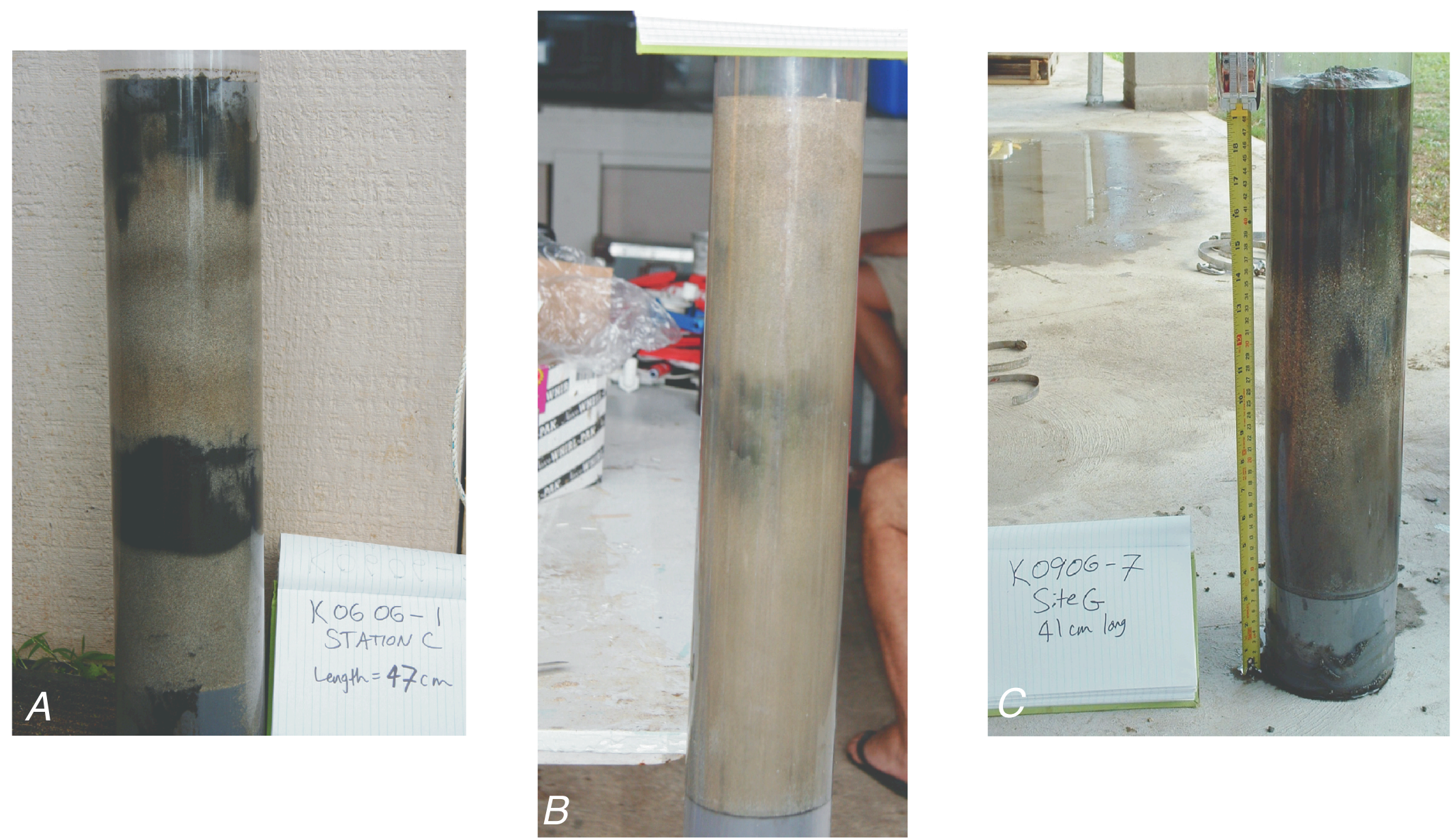

Figure 29. Photographs of sediment cores from Hanalei Bay. (A). Core collected at Site C in June 2006. Dark, muddy layers of sediment are deposits of terrestrial flood sediment. Light-colored carbonate sand occurs between the two flood layers and beneath the lower layer. The core length is $47 \mathrm{~cm}$. (B). Core collected at Site B in June 2006. Sediment in this core is dominated by marine carbonate sand. The core length is $53 \mathrm{~cm}$. (C). Core collected at Site $G$ in September 2006. The upper 20 cm of the core are dominated by dark, terrestrial sediment; below that, mixed mud and carbonate sand occur. The core length is $41 \mathrm{~cm}$. 
(activities of these isotopes, which sorb onto solid particles, decrease as the sediment grain size increases because the surface area per unit mass decreases as grain size increases).

Excess ${ }^{210} \mathrm{~Pb}$ inventory in the marine cores did not show patterns as clear as those of ${ }^{7} \mathrm{Be}$ and ${ }^{137} \mathrm{Cs}$. This is partially because cores were not always long enough to include all of the down-core extent of excess ${ }^{210} \mathrm{~Pb}$ (table 6). Although excess ${ }^{210} \mathrm{~Pb}$ profiles correspond with those of ${ }^{7} \mathrm{Be}$ and ${ }^{137} \mathrm{Cs}$ and thus are high in terrigenous sediment at some sites (such as in the uppermost $12 \mathrm{~cm}$ of Sites $\mathrm{A}$ and I in June 2006; figs. 5, 21), high excess ${ }^{210} \mathrm{~Pb}$ inventory measured at core sites away from the river mouth in 2005 suggests that scavenging from seawater is another significant source of excess ${ }^{210} \mathrm{~Pb}$ in Hanalei Bay sediment (Draut and others, 2006). None of the cores collected in 2005 or 2006 presented a "standard" excess ${ }^{210} \mathrm{~Pb}$ profile (one with a well defined surface mixed layer underlain by gradually decreasing activity and finally by background or "supported" activity) that would have characterized undisturbed sediment (Nittrouer and others, 1979). This is not surprising given the energetic wave climate in Hanalei Bay during the fall and winter that would be expected to resuspend sediment frequently; resuspension events would disturb any established ${ }^{210} \mathrm{~Pb}$ profile while also providing an opportunity for sediment to scavenge additional ${ }^{210} \mathrm{~Pb}$ from the water column. Given the history of frequent seasonal resuspension in the bay, it is not practical to estimate accumulation rate using geochemical models that are often applied to ${ }^{210} \mathrm{~Pb}$ profiles (Bentley and Nittrouer, 1999; Noller, 2000; Draut and others, 2005).

Fine-grained terrestrial sediment is commonly associated, in Hanalei Bay sediment cores, with organic debris including leaf litter, wood fragments, and roots that form a muddy, peat-like deposit. Occasional rounded siliciclastic pebbles were also recovered in marine sediment cores at the eastern side of the bay: at Site A in September 2006 (fig. 6) and at Sites A, F, and G in summer 2005 (Draut and others, 2006).

\section{Effects of the 2006 Hanalei River Floods in Hanalei Bay}

Deposits from the 2006 winter-spring floods provided the greatest terrestrial signal observed in the bay during this two-year study, leaving flood deposits in the eastern part of the bay that, as of June 2006, were on the order of $10 \mathrm{~cm}$ thick (thickest in the Black Hole bathymetric depression; Sites A, F, and I). Abundant wood and organic debris were also deposited on the sea floor in the eastern bay near the river mouth, in the Black Hole and along the eastern reef wall. Cores collected farther from the river mouth were dominated by carbonate sand with little or no ${ }^{7} \mathrm{Be}$ as of June 2006; carbonate facies were typical at Stations B, D, E, and H (fig. 2A) each time they were cored. The proportions of mud in seabed samples indicated that terrestrial mud did not decrease in a regular trend from east to west across the bay, but that the surface distribution of landderived mud was patchy (fig. 4). This patchiness is attributed to reworking of flood sediment by bottom currents and occasional wave activity during the summer months that redistributed sediment within the upper $\sim 5 \mathrm{~cm}$ of the sea floor (the thickness sampled by our seabed grab samples) across the bay without significantly affecting the deeper $(>10-\mathrm{cm}$ scale) stratigraphy. In spite of having undergone some reworking between the end of the spring floods (April 2006) and core collection, the sedimentary signal of the winter and spring floods was very apparent in June 2006. Seabed inventories of ${ }^{7} \mathrm{Be},{ }^{137} \mathrm{Cs}$, and excess ${ }^{210} \mathrm{~Pb}$ were typically much higher in June 2006 than at any other sampling time (table 6).

A Hanalei River flood occurred on August 7, 2006, that was smaller than floods the previous winter, but still a substantial discharge event for the summer season (fig. 3). Photographs taken by an automated camera station on the eastern side of the bay between Sites $\mathrm{C}$ and $\mathrm{G}$ (Outer Wall [OW] site in fig. 2A) showed that the August flood of $46 \mathrm{~m}^{3} \mathrm{~s}^{-1}$ deposited sediment on coral 
reefs in Hanalei Bay, as well as on the reef-free areas sampled by coring (fig. 30). Terrestrial sediment delivered by that flood was apparent in the Black Hole as of September 2006, but little sedimentary and isotopic evidence of the summer flood was present elsewhere in the bay by September. Stations A and I, both in the Black Hole, were the only two sites at which ${ }^{7}$ Be inventory in September 2006 was higher than could be accounted for simply by decay of the ${ }^{7}$ Be inventory that was present at those locations in June 2006. Stations A and I showed factors of 3 and 1.2, respectively, elevation in ${ }^{7} \mathrm{Be}$ inventory in September above the expected value from decay of the June inventory. This indicates the presence of new terrestrial sediment at those two sites, most of which presumably resulted from the flood on August 7. Magnetic properties shift at the same depth at which ${ }^{7} \mathrm{Be}$ appears in the September core at Site A $(\sim 10 \mathrm{~cm}$; figs. 6,24$)$, a shift that apparently reflects the August 2006 flood deposits. The flood deposits in the upper $10 \mathrm{~cm}$ in the September core at Site A (fig. 6) are similar to those in the June core at this site (fig. 5) but contained less magnetite and hematite and had finer magnetic grain size than those of the winter flood deposits (figs. 23, 24)-differences that, together with the ${ }^{7} \mathrm{Be}$ results, describe the presence of the summer flood sediment.

Deposits from the winter 2006 floods continued to dominate the sedimentary record in the eastern bay through early fall, even after the addition of newer sediment in the August 7 flood; this is consistent with the much higher sediment input of the winter floods compared with the August 7 flood (fig. 3). Peak daily water discharge in the largest winter flood (February 21, 2006) was a factor of 2.6 greater than that of the August 7 flood, while sediment concentration on that winter flood day exceeded the sediment concentration on August 7 by a factor of 5.9.

Sediment accumulating in the open center of the bay and on its fringing reefs is most readily remobilized and advected out of the bay during winter, when the wave climate is typically energetic. In summer, wave and current measurements made concurrently with this study (Storlazzi and others, 2006) showed weak currents $\left(\sim 1 \mathrm{~cm} \mathrm{~s}^{-1}\right.$ near the bed) and little wave energy, indicating that sediment delivered during summer floods most likely remains in the bay until winter storms can remove it. Increased turbidity and sedimentation on corals resulting from floods of the Hanalei River (as in fig. 30) could affect the sustainability of coral reefs and their many associated species. This is of particular concern during summer months when wave energy is low and sediment is not readily remobilized and advected out of the bay.

\section{Sources of Flood Sediment in the Hanalei Watershed}

To evaluate possible sources of terrestrial sediment in Hanalei Bay, magnetic properties and radioisotopes $\left({ }^{7} \mathrm{Be}\right.$ and $\left.{ }^{137} \mathrm{Cs}\right)$ of cored sediment in Hanalei Bay were compared with those of a variety of possible source materials at upland sites. Upland sampling sites included agricultural fields where taro is grown, steep banks of the Hanalei River, mud in minor drainages, poorly drained forest floor, sediment that was trapped by a grassy surface between the edge of the river and taro fields, and material on or adjacent to a dirt road parallel to the river. These settings do not encompass all possible sources in the watershed; for example, areas affected by feral ungulates and landslides on high-elevation, steep valley walls were not sampled.

Substantial variation in the concentrations of magnetite and hematite in the Hanalei Bay core sediment may have several causes: dilution by carbonate sediment, concentration in sediment plumes introduced to the bay related to initial sediment sources and to sorting during fluvial transport, and sorting during sediment transport within the bay water. Therefore, concentrations of these magnetic minerals cannot by themselves be used to identify source areas, or even whether there were many or few source areas. 

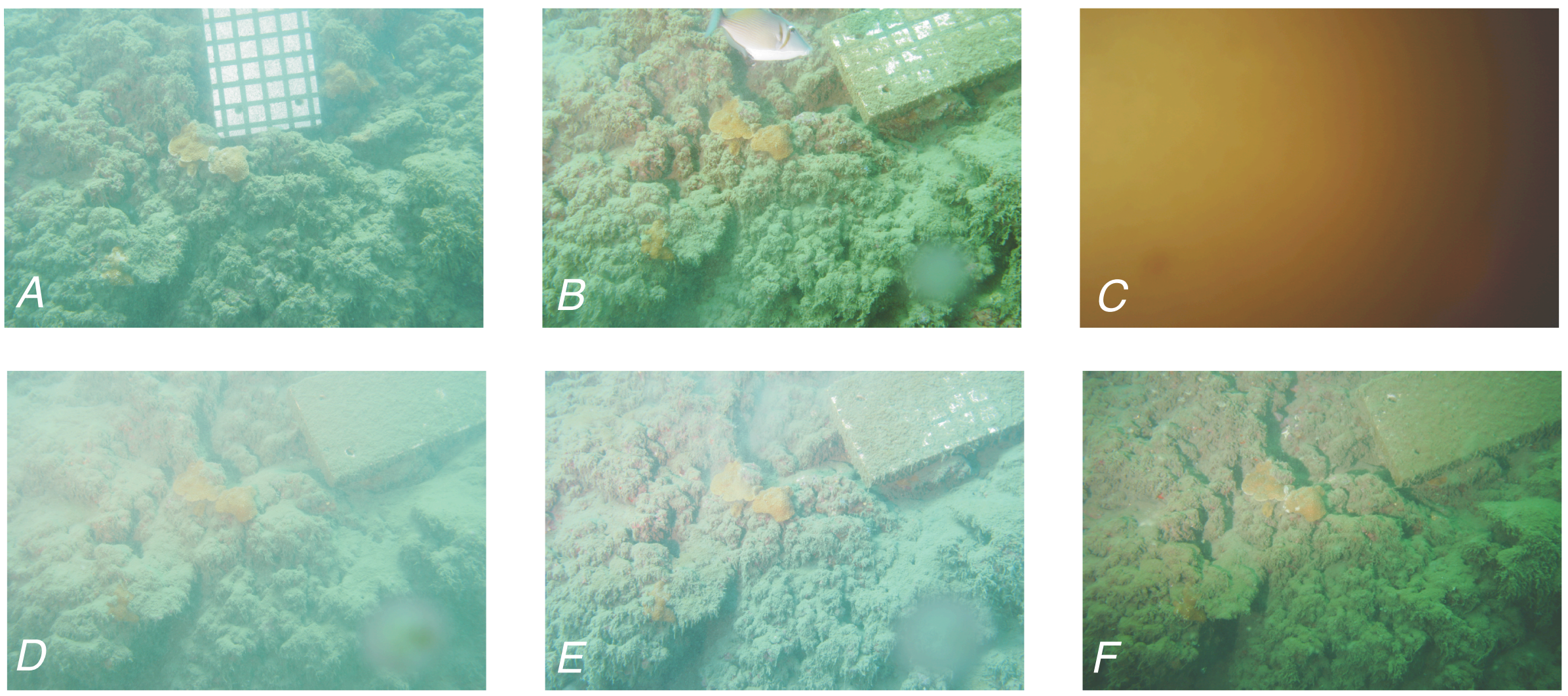

Figure 30. Photographs taken at an automated camera station in Hanalei Bay during the summer of 2006 . The camera was deployed on the sea floor facing a vertical coral-reef wall on the eastern side of Hanalei Bay (location in fig. 2). Sediment can be seen to accumulate on and erode off of the black and white grid and surrounding reef. (A). Photograph taken at 1259 on 6/7/06. (B). Photograph taken at 1700 on 8/2/06. (C). Photograph taken during a Hanalei River flood, at 1700 on 8/7/06. (D). Photograph taken several days after flood subsided, at 1300 on 8/11/06. (E). Photograph taken at 1700 on 8/16/06. (F). Photograph taken at 0900 on 9/7/06. All times listed are local (Hawaiian Standard Time). 
Broad variations in concentration-independent properties (relative magnetite-hematite abundance; magnetic grain size) with depth in all sampled bay sediments (cores from 2005 and 2006; appendix 3) indicate many sources of upland sediment. These variations can be used to infer some characteristics of sediment sources. The very large range of S-parameter values (0.74-0.98) represents large variation in relative amounts of magnetite and hematite in the bay sediment that is typical of multiple sources of terrigenous sediment having undergone different degrees of oxidation, probably related to the extent of surface weathering. All samples with very high hematite contents (taken here as $\mathrm{S}$ parameter $<0.90$ ) also have relatively high FDMS ( $>4$ percent). The combination of very low $\mathrm{S}$ parameter values and high FDMS strongly suggests that bay sediments with these properties were derived from deeply weathered rock or soil. The 19 samples having this combination of values (app. 3) contain very low ${ }^{137} \mathrm{Cs}$ activity (average $0.02 \mathrm{dpm} \mathrm{g}^{-1}$; undetected in most) and undetectable ${ }^{7} \mathrm{Be}$ activity with one exception $\left(0.05 \mathrm{dpm} \mathrm{g}^{-1}\right)$.

As stated in the preceding section, it is evident that flood deposits in the bay sediment have very tight ranges of ARM/MS, ARM/IRM, FDMS, and S-parameter values. These restricted properties suggest a limited number of possible sources, or sediments from many sources that are well mixed and stored at one or more locations in the watershed. Recent flood deposits have much higher ${ }^{137} \mathrm{Cs}$ activity levels (up to $1.76 \mathrm{dpm} \mathrm{g}^{-1}$, average $1.05 \mathrm{dpm} \mathrm{g}^{-1}$ ) than older terrestrial deposits (maximum of $0.46 \mathrm{dpm} \mathrm{g}^{-1}$; average of $0.06 \mathrm{dpm} \mathrm{g}^{-1}$ ). This observation indicates that recent floods have tapped, to great extent, sediment that was exposed to the atmosphere at some point within the past 40-60 years (based on ${ }^{137} \mathrm{Cs}$ activity) and also within the past 8 months (based on ${ }^{7} \mathrm{Be}$ activity). The link between high ${ }^{137} \mathrm{Cs}$ and flood deposits appears to be controlled by particle size: ${ }^{137} \mathrm{Cs}$ is relatively abundant in fine-grained core sediment, as it appears to be also in fine-grained watershed deposits, such as taro fields and recent flood sediment. Similar relations are found for ${ }^{7} \mathrm{Be}$ in these deposits.

No exact match is apparent among magnetic properties when comparing flood deposits in cores with upland samples (appendix 3). The closest similarities are found with sediment in taro fields (five samples) and in banks of the lower river (three samples). Taro-field samples had more hematite relative to magnetite (lower $\mathrm{S}$ parameter values) compared with flood deposits and, like the riverbank samples, had slightly coarser magnetic grain size (lower ARM/MS). Mineral sorting during transport in the lower reaches of the river during floods might explain the observed small discrepancies in magnetic grain-size parameters, assuming that our samples are representative of these landscape settings. Activity levels of ${ }^{137} \mathrm{Cs}$ in the taro-field samples $\left(0.79-1.69 \mathrm{dpm} \mathrm{g}^{-1}\right)$ partly span those of the flood deposits. Significant contribution to flood sediment from riverbanks, however, is precluded by very low ${ }^{137} \mathrm{Cs}$ activity in these settings compared with higher values of recent flood deposits in Hanalei Bay.

Taken together, magnetic and isotopic data suggest that a significant component of recent flood deposits in Hanalei Bay were derived from the floodplains of the lower watershed, where sediment from the upper watershed has accumulated and been stored. Stored sediment likely also has been well mixed during transport. The location or locations of such stored sediment are not known. Floodplain deposits in the taro fields show close (but not exact) affinity to the recent flood deposits in the Black Hole. Firm identification of sources for flood sediment awaits more investigation of possible sources, especially sediment that might be sequestered in fluvial deposits near the mouth of the river, in addition to more characterization of some flood components, especially organic matter. 
Finally, we have not obtained samples to evaluate directly any possible contributions from intermediate and high elevations $(>300 \mathrm{~m})$ that would include minimally reworked landslide debris and large areas disturbed by feral ungulates.

\section{Conclusions}

Sediment cores and seabed grab samples collected in Hanalei Bay, Kaua $i$, in June and September 2006 indicated that the youngest and thickest terrigenous sediment was present on the eastern side of the bay, near the Hanalei River mouth and in the Black Hole bathymetric depression, which acts as at least a temporary sediment sink. Floods that occurred between February and April 2006 left flood deposits in the eastern bay that, by June 2006, were on the order of $10 \mathrm{~cm}$ thick. A flood occurred on August 7, 2006, that was smaller than floods that occurred during the previous winter, but was still a substantial discharge event for the summer season, and of similar magnitude to summer floods that occurred in 2000 and 2003. Deposits from the 2006 winter-spring floods dominated the sedimentary record in the eastern bay through early fall, even after the addition of newer sediment by the August 7 flood; this is consistent with the much higher sediment input of the winter floods compared with the August 7 flood.

Variations in magnetic grain size and in the relative magnetite-hematite abundance in sediment cores indicate many sources of upland terrigenous sediment. As a group, recent (2006) flood deposits show much less variation in these properties compared with older deposits. Sediment delivered by the August 2006 flood was apparently derived from source material with a somewhat finer magnetic grain size than sediment delivered by the winter-spring floods. Magnetic properties of flood deposits imply either that the 2006 winter-spring flood sediment was sourced from one or a few distinct upland settings, or that substantial mixing of sediment from multiple sources occurred during transport in flood waters.

The coral-reef community in Hanalei Bay has the potential to be adversely affected by the delivery of fluvial sediment. Deposition on and burial of corals by sediment during summer floods, when wave and current energy are low and sediment is not readily remobilized, could have significant effects on Hawaiian reef ecosystems, especially as coral spawning typically occurs between June and August. Therefore, the timing (seasonality) and magnitude of sediment input to the coastal ocean, relative to seasonal variations in wave and current energy, could have significant ecological consequences for coral reefs in the Hawaiian Islands.

\section{Acknowledgments}

This project was supported as part of the USGS Coral Reef Project. We thank Carl Berg, Chief Scientist, Hanalei Watershed Hui, for valuable discussions and guidance, and for logistical help in the field. Ron Hoeke, Curt Storlazzi, Kathy Presto, and Thomas Reiss assisted with field work. Charles Bass, Garrett Santos, Ryan Siebring, and Earl Loder are thanked for boat operation and assistance. 


\section{References}

Allison, M.A., Kuehl, S.A., Martin, T.C., and Hassan, A., 1998, Importance of flood-plain sedimentation for river sediment budgets and terrigenous input to the oceans - insights from the Brahmaputra-Jamuna River: Geology, v. 26, p. 175-178.

Allison, M.A., Kineke, G.C., Gordon, E.S., and Goñi, M.A., 2000, Development and reworking of a seasonal flood deposit on the inner continental shelf off the Atchafalaya River: Continental Shelf Research, v. 20, p. 2267-2294.

Baskaran, M., Coleman, C.H., and Santschi, P.H., 1993, Atmospheric depositional fluxes of ${ }^{7}$ Be and ${ }^{210} \mathrm{~Pb}$ at Galveston and College Station, Texas: Journal of Geophysical Research, v. 98, p. 20,555-20,571.

Bentley, S.J., and Nittrouer, C.A., 1999, Physical and biological influences on the formation of sedimentary fabric in an oxygen-restricted depositional environment, Eckenförde Bay, southwestern Baltic Sea: Palaios, v. 14, p. 585-600.

Bothner, M.H., Reynolds, R.L., Casso, M.A., Storlazzi, C.D., and Field, M.E., 2006, Quantity, composition, and source of sediment collected in sediment traps along the fringing coral reef off Molokai, Hawaii: Marine Pollution Bulletin, v. 52, p. 1034-1047.

Buesseler, K., and Benitez, C., 1994, Determination of mass accumulation rates and sediment radionuclide inventories in the deep Black Sea: Deep-Sea Research Part I, v. 41, p. 1605-1615.

Calhoun, R.S., and Fletcher, C.H., 1996, Late Holocene coastal plain stratigraphy and sea-level history at Hanalei, Kauai, Hawaiian Islands: Quaternary Research, v. 45, p. 47-58.

Calhoun, R.S., and Fletcher, C.H., 1999, Measured and predicted sediment yield from a subtropical, heavy rainfall, steep-sided river basin-Hanalei, Kauai, Hawaiian Islands: Geomorphology, v. 30, p. 213-226.

Calhoun, R.S., Fletcher, C.H., and Harney, J.N., 2002, A budget of marine and terrigenous sediments, Hanalei Bay, Kauai, Hawaiian Islands: Sedimentary Geology, v. 150, p. 61-87.

Canuel, E.A., Martens, C.S., and Benninger, L.K., 1990, Seasonal variations in ${ }^{7}$ Be activity in the sediments of Cape Lookout Bight, North Carolina: Geochimica et Cosmochimica Acta, v. 54, p. 237-245.

Carver, R.E., 1971, Procedures in sedimentary petrography: New York, John Wiley and Sons, 653 p.

Crusius, J., Bothner, M.H., and Sommerfield, C.K., 2004, Bioturbation depths, rates, and processes in Massachusetts Bay sediments inferred from modeling of ${ }^{210} \mathrm{~Pb}$ and ${ }^{239+240} \mathrm{Pu}$ profiles: Estuarine, Coastal, and Shelf Science, v. 61, p. 643-655. 
Cutshall, N.H., Larsen, I.L., and Olsen, C.R., 1983, Direct analyses of ${ }^{210} \mathrm{~Pb}$ in sediment samples - self-absorption corrections: Nuclear Instruments and Methods, v. 26, p. 309-312.

Draut, A.E., Kineke, G.C., Velasco, D.W., Allison, M.A., and Prime, R.J., 2005, Influence of the Atchafalaya River on recent evolution of the chenier-plain inner continental shelf, northern Gulf of Mexico: Continental Shelf Research, v. 25, p. 91-112.

Draut, A. E., Field, M. E., Bothner, M. H., Logan, J. B., Casso, M. A., Baldwin, S. M., and Storlazzi, C. D., 2006, Coastal circulation and sediment dynamics in Hanalei Bay, Kaua'i, Hawai'i, Part II, tracking recent fluvial sedimentation - Isotope stratigraphy obtained in summer 2005: U.S. Geological Survey Open-File Report 2006-1125, 52 p.

Duursma, E.K., and Gross, M.G., 1971, Marine sediments and radioactivity, in Radioactivity in the marine environment, Panel on Radioactivity in the Marine Environment of the Committee on Oceanography, National Research Council: Washington, D.C., National Academy of Sciences, p. $147-160$.

Fabricius, K.E., and Wolanski, E., 2000, Rapid smothering of coral reef organisms by muddy marine snow: Estuarine, Coastal and Shelf Science, v. 50, p. 115-120.

Folk, R.L., 1974, Petrology of sedimentary rocks: Austin, University of Texas Publication, 170 p.

Friedlander, A.M., DeFelice, R.C., Parrish, J.D., and Frederick, J.L., 1997, Habitat resources and recreational fish populations at Hanalei Bay, Kauai: Final Report submitted to the Hawai'i Department of Land and Natural Resources, Division of Aquatic Resources, 320 p.

Friedlander, A.M., Brown, E.K., and Helweg, D.A., 2005, Hanalei Bay marine benthic communities since 1992-Spatial and temporal trends in a dynamic Hawaiian coral reef ecosystem. Hawai'i Cooperative Studies Unit Technical Report, Hawai'i Cooperative Studies Unit, Pacific Aquaculture and Coastal Resources Center, University of Hawai'i at Hilo.

Gäggler, H., Von Gunten, H.R., and Nyffeler, V., 1976, Determination of 210Pb in lake sediments and air samples by direct gamma-ray measurement: Earth and Planetary Science Letters, v. 33, p. 119-121.

Goodbred, S.L., Jr., and Kuehl, S.A., 1998, Floodplain processes in the Bengal Basin and storage of Ganges-Brahmaputra river sediment-An accretion study using ${ }^{137} \mathrm{Cs}$ and ${ }^{210} \mathrm{~Pb}$ geochronology: Sedimentary Geology, v. 121, p. 239-258.

Jaeger, J.M., and Nittrouer, C.A., 1995, Tidal controls on the formation of fine-scale sedimentary strata near the Amazon mouth: Marine Geology, v. 125, p. 259-281.

Joshi, S.R., 1987, Nondestructive determination of Lead-210 and Radium-226 in sediments by direct photon analysis: Journal of Radioanalytical and Nuclear Chemistry, v. 116, p. 169-212. 
Kuehl, S.A., Pacioni, T.D., and Rine, J.M., 1995, Seabed dynamics of the inner Amazon continental shelf - temporal and spatial variability of surficial strata: Marine Geology, v. 125, p. 283-302.

Livingston, H.D., and Bowen, V.T., 1979, Pu and 137Cs in coastal sediments: Earth and Planetary Science Letters, v. 43, p. 29-45.

Miller, K.M., and Heit, M., 1986, A time resolution methodology for assessing the quality of lake sediment cores that are dated by 137Cs: Limnology and Oceanography, v. 31, p. 1292-1300.

Moberly, R.M., and Chamberlain, T., 1964, Hawaiian Beach Systems: University of Hawai'i, 95 p.

Nittrouer, C.A., Sternberg, R.W., Carpenter, R., and Bennett, J.T., 1979, The use of Pb-210 geochronology as a sedimentary tool-Application to the Washington continental shelf: Marine Geology, v. 31, p. 297-316.

Noller, J.S., 2000, Lead-210 Geochronology, in Quaternary Geochronology, Methods and Applications: American Geophysical Union Reference Shelf No. 4, p. 115-120.

Olsen, C.R., Larsen, I.L., Lowry, P.D., and Cutshall, N.H., 1986, Geochemistry and deposition of ${ }^{7} \mathrm{Be}$ in river-estuarine and coastal waters: Journal of Geophysical Research, v. 91, p. 896-908.

Reynolds, R.L., Rosenbaum, J.G., Hudson, M.R., and Fishman, N.S., 1990, Rock magnetism, the distribution of magnetic minerals in the Earth's crust, and aeromagnetic anomalies, in Hanna, W.F., ed., Proceedings of a Workshop in Geologic Applications of Modern Aeromagnetic Surveys: U.S. Geological Survey Bulletin 1924, p. 24-45.

Rogers, C.S., 1990, Responses of coral reefs and reef organisms to sedimentation: Marine Ecology Progress Series 62, p. 185-202.

Rosenbaum, J.G., Reynolds, R.L., and Schlinger, C.M., 1991, Effects of cooling on oxide mineralogy and magnetic properties near margins of volcanic bodies: EOS, American Geophysical Union Transactions, v. 72, p. 138.

Rosenbaum, J.G., Reynolds, R.L., and Hildenbrand, T., 1994, Insight into the structure of the East Rift Zone of Kilauea, Hawaii, from a rock magnetic study of drill core: VIIth International Drilling Symposium Proceedings, p. 142-145.

Smith, J.N., and Ellis, K.M., 1982, Transport mechanism for Pb-210, Cs-137 and Pu fallout radionuclides through fluvial-marine systems: Geochimica et Cosmochimica Acta, v. 46, p. 941-954.

Storlazzi, C.D., Presto, M.K., Logan, J.B., and Field, M.E., 2006, Coastal circulation and sediment dynamics in Hanalei Bay, Kaua'i, Part I-Measurements of waves, currents, temperature, salinity and turbidity, June-August, 2005: U.S. Geological Survey Open-File Report 2006-1085. 
1. Sediment log for cores and river-bed samples collected in June 2006

Station A (Core K0606-A(2); Black Hole):

0-6 cm: Unconsolidated, black-brown mud with organic debris including leaves and twigs. This unit has a sharp lower contact.

6-12 cm: Carbonate sand (dominant) with dark mud.

$12-30 \mathrm{~cm}$ : Gradational transition to more black-gray mud with sparse carbonate sand and sparse organics.

30-55 cm: Black-brown mud, sparse carbonate sand, occasional coral rubble.

Station B (Core K0606-B(3); South-central bay):

0-20 cm: Carbonate-dominated sand. No surface "fluff" layer.

20-24 cm: Slightly muddier, darker, but still mostly carbonate sand. No well defined contacts or horizons.

24-53 cm: Carbonate-dominated sand.

Station C (Core K0606-C(1); NE side of bay along coral wall):

0-2 cm: Dark brown-black mud.

2-28 cm: Carbonate-dominated sand with sparse mud.

28-34 cm: Black, consolidated, very fine mud.

34-47 cm: Homogenous carbonate-dominated sand.

Station D (Core K0606-D(4); NW side of bay near CRAMP site):

0-35 cm: Unconsolidated, carbonate-dominated sand; homogenized during core collection. No surface fluff layer at site.

Station E (Core K0606-E(6); Middle of bay):

0-44.5 cm: Carbonate-dominated sand with no visible stratification.

Station F (Core K0606-F(7); Black Hole):

0-12 cm: Black, very unconsolidated mud. Twigs, organic material. Essentially no sand noted.

12-16 cm: Carbonate-dominated sand mixed with mud.

16-22 cm: Mixed sand and mud, dominated by black mud.

22- 54 cm: Less sand than overlying horizon; black and brown sticky, consolidated mud. Contains terrigenous leaves, wood. Gradual transition downward into mixed sand and mud (at $30-32 \mathrm{~cm}$ ) but still has leaves, organic debris. Sulfurous smell.

54-57 cm: Gray, well consolidated clay with little sand.

Station G (Core K0606-G(5); Along reef wall near Hanalei River mouth):

0-3 cm: "Fluffy" unconsolidated black-brown mud.

3-12 cm: Mixed carbonate sand and mud. Better consolidated than overlying horizon. 
12-16 cm: Over this depth a gradual downward transition occurs to higher mud content (mixed sand and mud) below; the mud is black with organic debris, plant fibers.

16-32 cm: Muddy carbonate sand with sharp lower contact.

$32-46 \mathrm{~cm}$ : Carbonate sand with less mud than overlying unit.

Station H (Core K0606-H(8); East side of bay, South of Black Hole):

Minor surface "fluff" layer.

0-39 cm: Carbonate-dominated sand with no distinct stratification.

Core homogenized somewhat during collection, but no strata were visible before mixing.

Station I (Core K0606-I(9); NE side of bay, along wall, between Stations C and $\mathbf{G ) :}$

0-10 cm: Black, very unconsolidated mud. Rare organics (roots, etc.).

10-14 cm: Over this depth is a gradual downward transition to mud that includes sparse sand. Mud is black, with organic matter.

14-26 cm: Mixed sand and mud. The base of the upper mud unit is at $14 \mathrm{~cm}$, but the contact is not sharp. Sand is visibly bioturbated, with mud-filled burrows. Some larger coral and shell fragments are apparent. The base of this unit is coarse shell hash.

26-32 cm: Black, sticky mud with some woody debris. There is a sharp contact at $32 \mathrm{~cm}$ between upper, black woody mud and lower brown clayey mud, with a distinct color change.

32-55 cm: Brown, uniform, clay-rich mud. Sand and organic matter are absent.

River Site 1 ( $\sim 4 \mathrm{~km}$ upstream of river mouth, near USGS gaging station 16103000): Sediment at this site consisted of some consolidated mud forming the river bank, and sandier sediment in the channel bottom. Leaves and woody debris are abundant.

River Site 2 (under the traffic bridge where the access road between the towns of Hanalei and Princeville crosses the Hanalei River, $\sim 2.5 \mathrm{~km}$ above the river mouth): Sediment at this site is predominantly mud with abundant organic debris.

River Site 3 (approximately $\mathbf{0 . 5} \mathbf{~ k m}$ above the river mouth): This site, sampled in August 2005 (Draut and others, 2006), was not sampled in 2006.

River Site 4 (on river left at the river mouth, adjacent to the Hanalei beach park): Sediment at this site is fine to medium sand and mud that forms a sandbar 10-20 m wide. 


\section{Sediment log for cores and river-bed samples collected in September 2006}

Station A (Core K0906-A(5); Black Hole):

0-10 cm: Black, very unconsolidated mud with thick surface "fluff" layer. This unit is bioturbated with a large worm present in the core. At $10 \mathrm{~cm}$ there is a sharp contact.

10-14 cm: Mixed carbonate-dominated sand and mud, better consolidated than the overlying unit. Leaves and twigs occur at $10 \mathrm{~cm}$; a terrestrial pebble is present at $12 \mathrm{~cm}$.

14-30 cm: Fine brown/black mud, fairly consolidated, with little carbonate sand. Burrows are visible. The lower contact of this unit is gradational.

30-59 cm: Mixture of consolidated gray-brown mud and carbonate-dominated sand. Leaf debris is present at $42 \mathrm{~cm}$. From $50-59 \mathrm{~cm}$ the sediment produced a strong smell of sulfur.

Station B (Core K0906-B(2); South-central bay):

Very thin surface "fluff" layer.

0-46 cm: Carbonate-dominated sand. Sediment is fairly homogenous but with mottling and bioturbation apparent in the uppermost $4 \mathrm{~cm}$. No visible stratification.

Station C (Core K0906-C(4); NE side of bay along coral wall):

$0-46 \mathrm{~cm}$ : Mixed carbonate and terrigenous sand.

$46-49 \mathrm{~cm}$ : Black mud unit.

49-51 cm: Mixed carbonate and terrigenous sand.

Station D (Core K0906-D(1); NW side of bay near CRAMP site):

0-34 cm: Unconsolidated, homogenous, carbonate-dominated sand.

Station E (Core K0906-E(3); Middle of bay):

Very thin surface "fluff" layer present.

0-46 cm: Homogenous, carbonate-dominated sand with no visible stratification.

Station F (Core K0906-F(6); Black Hole):

0-12 cm: Very unconsolidated brown-black mud with a sharp lower contact.

Twigs and other woody debris (stem-sized) are present in uppermost unit.

12- 19 cm: Consolidated mixture of mud and carbonate-dominated sand.

Bioturbated (worm present). Lower contact is gradational and spans $18-20 \mathrm{~cm}$ depth.

19-67 cm: Consolidated, stiff black-brown mud unit with minor carbonate sand.

Burrows present at $\sim 35 \mathrm{~cm}$. Woody debris present at $\sim 40-50 \mathrm{~cm}$ and again at $55 \mathrm{~cm}$. Lower contact of this unit is gradational over $\sim 1 \mathrm{~cm}$.

67-72 cm: Stiff, clayey brown mud with little or no sand. 
Station G (Core K0906-G(7); Along reef wall near Hanalei River mouth): 0-4 cm: Poorly consolidated brown-black mud with trace carbonate sand. $4-8 \mathrm{~cm}$ : Mixed sand (carbonate-dominated) and mud. Lower contact is gradational.

8-41 cm: Coarse carbonate-dominated sand with minor black mud mixed in. Mottled/bioturbated with worm present. At $35 \mathrm{~cm}$ a possible mud rip-up clast is present, $3 \mathrm{~cm}$ in diameter. At $40 \mathrm{~cm}$ there is terrestrial plant matter.

Station H (Core K0906-H(9); East side of bay, South of Black Hole): Surface 'fluff' layer a few mm thick.

0-48 cm: Sand, mostly carbonate. From 4-10 cm is slightly darker sand, but it still appears to be mixed carbonate and terrestrial sand. Lower contact (at $48 \mathrm{~cm}$ ) is sharp.

48-60.5 cm: Dark gray, sandy mud. Faint sulfur smell; no wood or leaves apparent.

Station I (Core K0906-I(8); NE side of bay, along wall, between Stations C and $\mathbf{G ) :}$

0-53 cm: Very unconsolidated black mud; extremely soft sea floor. This facies is present throughout the core. Consolidation gradually increases down-core but sediment is still very poorly consolidated all the way to the core base. Sediment is very dark black, with occasional rootlets and an organic smell.

River Site 1 ( $4 \mathrm{~km}$ upstream of river mouth, near USGS gaging station 16103000): Sediment consisted of consolidated mud forming the river bank, with sandier sediment in the channel bottom. Leaves and woody debris are abundant.

River Site 2 (under the traffic bridge where the access road between the towns of Hanalei and Princeville crosses the Hanalei River, $\sim 2.5 \mathrm{~km}$ above the river mouth): Sediment at this site is dominantly consolidated mud forming the river banks, with abundant organic debris.

River Site $\mathbf{3}$ (approximately $\mathbf{0 . 5} \mathbf{~ k m}$ above the river mouth): This site, sampled in August 2005 (Draut and others, 2006), was not sampled in 2006.

River Site 4 (on river left at the river mouth, adjacent to the Hanalei beach park): Sediment at this site is fine to medium sand and sparser mud, forming a sandbar 10-20 $\mathrm{m}$ wide. 